CRYSTALLOGRAPHIC COMMUNICATIONS

ISSN 2056-9890

Received 18 October 2021

Accepted 29 October 2021

Edited by A. M. Chippindale, University of Reading, England

Keywords: caesium lutetium(III) silicate; crystal structure; single-chain silicate.

CCDC reference: 2118472

Supporting information: this article has supporting information at journals.iucr.org/e

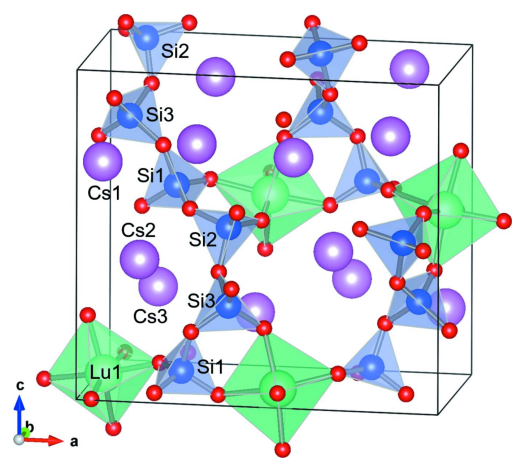

OPEN $\odot$ ACCESS

\section{Crystal structure of chain silicate $\mathrm{Cs}_{3} \mathrm{LuSi}_{3} \mathrm{O}_{9}$}

\author{
Hiromitsu Kimura $^{a}$ and Hisanori Yamane ${ }^{b_{*}}$
}

a Inorganic Materials Laboratory, Science \& Innovation Center, Mitsubishi Chemical Corporation, 1000 Kamoshida-cho, Aoba-ku, Yokohama-shi, Kanagawa, 227-8502, Japan, and ${ }^{\mathbf{b}}$ Institute of Multidisciplinary Research for Advanced Material, Tohoku University, 2-1-1 Katahira, Aoba-ku, Sendai, Miyagi, 980-8577, Japan. *Correspondence e-mail: hisanori.yamane.a1@tohoku.ac.jp

A caesium lutetium(III) silicate, $\mathrm{Cs}_{3} \mathrm{LuSi}_{3} \mathrm{O}_{9}$, was synthesized by heating a pelletized mixture of $\mathrm{Cs}_{2} \mathrm{CO}_{3}, \mathrm{Lu}_{2} \mathrm{O}_{3}$ and $\mathrm{SiO}_{2}$ at $1273 \mathrm{~K}$. Single crystals of the title compound were grown in a melted area of the pellet. $\mathrm{Cs}_{3} \mathrm{LuSi}_{3} \mathrm{O}_{9}$ is a singlechain silicate (orthorhombic space group $P n a 2_{1}$ ) with a chain periodicity of six and is isostructural with $\mathrm{Cs}_{3} R E^{\mathrm{II}} \mathrm{Ge}_{3} \mathrm{O}_{9}(R E=\mathrm{Pr}, \mathrm{Nd}$ and $\mathrm{Sm}-\mathrm{Yb})$. The two symmetry-dependent $\left[\mathrm{Si}_{6} \mathrm{O}_{18}\right]^{12-}$ chains in the unit cell lie parallel to the [011] direction. The $\mathrm{Lu}^{3+}$ ions are octahedrally coordinated by $\mathrm{O}$ atoms of the silicate chains, generating a three-dimensional framework. $\mathrm{Cs}^{+}$ions are located in the voids in the framework.

\section{Chemical context}

A lutetium(III) silicate, $\mathrm{Lu}_{2} \mathrm{SiO}_{5}$, containing the highest atomic weight rare-earth element, has been studied as the host crystal of a scintillator for radiation detection (Dorenbos et al., 1994; Melcher \& Schweitzer, 1992). Our research group recently reported the syntheses and crystal structures of a new oxide and oxynitride containing $\mathrm{Lu}$; namely, $\mathrm{Lu}_{4} \mathrm{Al}_{2} \mathrm{O}_{9}$ (Simura \& Yamane, 2020a) and $\mathrm{Ba}_{0.9} \mathrm{Ce}_{0.1} \mathrm{LuAl}_{0.2} \mathrm{Si}_{3.8} \mathrm{~N}_{6.9} \mathrm{O}_{0.1}$ (Simura \& Yamane, 2020b). In the present study, a new quaternary single-chain silicate, $\mathrm{Cs}_{3} \mathrm{LuSi}_{3} \mathrm{O}_{9}$, was found during an exploratory study of the $\mathrm{Cs}-\mathrm{Lu}-\mathrm{Si}-\mathrm{O}$ system. In other quaternary silicate systems containing alkali-metal $(A)$ and rare-earth $(R E)$ elements, a number of single-chain silicates have recently been reported: $\mathrm{LiScSi}_{2} \mathrm{O}_{6}$ (space groups $C 2 / c$, $P 2_{1} / c$; Arlt \& Angel, 2000; Redhammer \& Roth, 2004), $\mathrm{NaYSi}_{2} \mathrm{O}_{6}\left(P 2_{1} / c\right.$; Többens et al., 2005), $\mathrm{NaTbSi}_{2} \mathrm{O}_{6}\left(P 2_{1} / c\right.$; Schäfer et al., 2012), $\mathrm{Na}_{3} \mathrm{YSi}_{3} \mathrm{O}_{9}\left(P 2_{1} 2_{1} 2_{1}\right.$; Maksimov et al., 1980), $\mathrm{Na}_{3} R E \mathrm{Si}_{3} \mathrm{O}_{9}\left(R E=\mathrm{Y}, \mathrm{Dy}, \mathrm{Gd}, P 2_{1} 2_{1} 2_{1}\right.$; Shannon et al., 1980), $\mathrm{Na}_{3} R E \mathrm{Si}_{3} \mathrm{O}_{9}\left(R E=\mathrm{Y}, \mathrm{Er}, P 2_{1} 2_{1} 2_{1}\right.$; Ananias et al., 2002), $\mathrm{Na}_{3} \mathrm{TmSi}_{3} \mathrm{O}_{9}\left(P 2_{1} 2_{1} 2_{1}\right.$; Kahlenberg et al., 2015), $\mathrm{K}_{3} \mathrm{HoSi}_{3} \mathrm{O}_{9}$ $\left(P m 2_{1} n\right.$; Ponomarev et al., 1988), $\mathrm{K}_{3} R E \mathrm{Si}_{3} \mathrm{O}_{9}(R E=\mathrm{Ho}, \mathrm{Tm}$, $\mathrm{Lu}, P m 2_{1} n$; Filipenko et al., 1988), $\mathrm{K}_{3} \mathrm{TbSi}_{3} \mathrm{O}_{9}\left(P m 2_{1} n\right.$; Kostova et al., 2007), and $\mathrm{K}_{2.9} \mathrm{Rb}_{0.1} \mathrm{ErSi}_{3} \mathrm{O}_{9}(P 1$; WierzbickaWieczorek et al., 2010). $\mathrm{Na}^{+}$ion conduction and photoluminescence have been investigated on doping $\mathrm{Eu}, \mathrm{Tb}$ and Tm into $\mathrm{Na}_{3} \mathrm{YSi}_{3} \mathrm{O}_{9}$ (Shannon et al., 1980; Kim et al., 1985; Banks \& Kim, 1985; Ananias et al., 2006; Zhang et al., 2008a,b). Photoluminescence properties have also been characterized for $\mathrm{Na}_{3} \mathrm{ErSi}_{3} \mathrm{O}_{9}$ (Ananias et al., 2002) and $\mathrm{K}_{3} R E \mathrm{Si}_{3} \mathrm{O}_{9}(R E=$ $\mathrm{Y}, \mathrm{Eu}, \mathrm{Tb}$; Kostova et al., 2007). A series of germanates with a new structure type, $\mathrm{Cs}_{3} R E^{\mathrm{III}} \mathrm{Ge}_{3} \mathrm{O}_{9}(R E=\mathrm{Pr}, \mathrm{Nd}$ and $\mathrm{Sm}-\mathrm{Yb}$, space group $P n a 2_{1}$ ) have recently been reported (Morrison et al., 2019). In the present study, we report the synthesis and structural characterization of $\mathrm{Cs}_{3} \mathrm{LuSi}_{3} \mathrm{O}_{9}$, the first silicate found to be isostructural with the germanates. 


\section{Structural commentary}

$\mathrm{Cs}_{3} \mathrm{LuSi}_{3} \mathrm{O}_{9}$ is a sechser single-chain silicate, which crystallizes in the orthorhombic space group, Pna2 ${ }_{1}$. The chains of Si1-, $\mathrm{Si} 2$ - and $\mathrm{Si} 3$-centered oxygen tetrahedra are aligned along the [110] direction (Figs. 1 and 2). The period of the $\mathrm{SiO}_{4}$ zigzag chain is $\mathrm{Si1}-\mathrm{Si2}-\mathrm{Si3}-\mathrm{Si} 1-\mathrm{Si2}-\mathrm{Si3}$, and the chain can be described as an unbranched $(\boldsymbol{u} \boldsymbol{B})$ single chain $1^{1} \infty$ with a six$\left[\mathrm{SiO}_{4}\right]$-tetrahedra repeat unit $\left.\left[{ }^{6} \mathrm{Si}_{6} \mathrm{O}_{18}\right]:\left\{\mathrm{uB}, 1_{\infty}^{1}\right\}\right\}\left[{ }^{6} \mathrm{Si}_{6} \mathrm{O}_{18}\right]$, in accordance with the classification of Liebau (1985). The Si$\mathrm{O}_{\text {bridge }}$ bond lengths in the title compound lie in the range 1.650 (4) -1.664 (4) $\AA$, and are slightly longer than the $\mathrm{Si}-$ $\mathrm{O}_{\text {terminal }}$ bond lengths [in the range 1.594 (4)-1.609 (4) $\AA$; Table 1) and are in agreement with values found in other silicates. The bond-valence sums (BVSs) calculated for Si1, $\mathrm{Si} 2$, and $\mathrm{Si} 3$ using the bond-valence parameters presented by Gagné \& Hawthorne (2015), are 3.903, 3.966, and 3.948, respectively, which closely match the value of 4 expected for $\mathrm{Si}^{\mathrm{IV}}$.

The angles $\mathrm{Si} 1-\mathrm{Si} 2-\mathrm{Si} 3, \mathrm{Si} 2-\mathrm{Si} 3-\mathrm{Si} 1$ and $\mathrm{Si} 3-\mathrm{Si} 1-\mathrm{Si} 2$ in the $\mathrm{SiO}_{4}$-linked zigzag chain in $\mathrm{Cs}_{3} \mathrm{LuSi}_{3} \mathrm{O}_{9}$ are 133.26 (7), $128.41(7)$ and $134.64(7)^{\circ}$, respectively. These values are larger than the angles observed in $\mathrm{K}_{6} \mathrm{Lu}_{2} \mathrm{Si}_{6} \mathrm{O}_{18}$ (space group $P m 2_{1} n$, Filipenko et al., 1988), a sechser single-chain silicate with an Si1-Si2-Si3-Si3-Si2-Si1 sequence (and angles: $\mathrm{Si} 1-$ $\mathrm{Si} 2-\mathrm{Si} 3=80.45, \mathrm{Si} 2-\mathrm{Si} 3-\mathrm{Si} 3=123.65$ and $\mathrm{Si} 3-\mathrm{Si} 3-\mathrm{Si} 2=$ $123.65^{\circ}$ ), reflecting the difference in ionic size between $\mathrm{Cs}^{+}$ and $\mathrm{K}^{+}$.

Lu1 has sixfold coordination and is located in a distorted $\mathrm{O}$ octahedron, which connects two $\mathrm{SiO}_{4}$ single chains, as shown in Fig. 2. All the oxygen atoms of the Lu1-based octahedron are shared with $\mathrm{SiO}_{4}$ tetrahedra. The $\mathrm{Lu} 1-\mathrm{O}$ bond distances range from 2.203 (4) to 2.255 (4) $\AA$, and the average distance $(2.2282 \AA)$ is in good agreement with the corresponding value reported for $\mathrm{K}_{6} \mathrm{Lu}_{2} \mathrm{Si}_{6} \mathrm{O}_{18}$ (Filipenko et al., 1988). A BVS value of 2.939 was obtained, which is close to the valence of 3 expected for $\mathrm{Lu}^{\mathrm{III}}$.



Figure 1

Atomic arrangement of $\mathrm{Cs}_{3} \mathrm{LuSi}_{3} \mathrm{O}_{9}$ shown with displacement ellipsoids at the $99 \%$ probability level. [Symmetry codes: (i) $-x+\frac{1}{2}, y+\frac{1}{2}, z+\frac{1}{2}$; (ii) $-x,-y, z+\frac{1}{2}$; (iii) $x+\frac{1}{2},-y+\frac{1}{2}, z$; (iv) $x, y, z+1$; (v) $-x+\frac{1}{2}, y-\frac{1}{2}, z+\frac{1}{2}$; (vi) $-x, 2-y, z+\frac{1}{2}$; (vii) $x-\frac{1}{2},-y+\frac{1}{2}, z$; (viii) $x, y+1, z$; (ix) $-x+\frac{1}{2}, y-\frac{1}{2}, z-\frac{1}{2}$; (x) $-x, 1-y, z-\frac{1}{2}$; (xi) $\left.x, y, z-1\right]$.
Table 1

Selected bond lengths $(\AA)$.

\begin{tabular}{|c|c|c|c|}
\hline $\mathrm{Cs} 1-\mathrm{O} 3$ & $2.869(4)$ & $\mathrm{Cs} 3-\mathrm{O} 5$ & 3.057 (4) \\
\hline $\mathrm{Cs} 1-\mathrm{O} 4^{\mathrm{i}}$ & $2.972(4)$ & $\mathrm{Cs} 3-\mathrm{O} 4$ & $3.066(4)$ \\
\hline $\mathrm{Cs} 1-\mathrm{O} 1^{\mathrm{ii}}$ & $3.028(4)$ & $\mathrm{Cs} 3-\mathrm{O}^{\text {viii }}$ & $3.335(5)$ \\
\hline $\mathrm{Cs} 1-\mathrm{O}^{\mathrm{iii}}$ & 3.069 (4) & $\mathrm{Cs} 3-\mathrm{O} 8$ & $3.354(4)$ \\
\hline $\mathrm{Cs} 1-\mathrm{O} 2^{\mathrm{ii}}$ & $3.074(5)$ & $\mathrm{Cs} 3-\mathrm{O} 6$ & $3.599(5)$ \\
\hline $\mathrm{Cs} 1-\mathrm{O}^{\mathrm{iv}}$ & 3.485 (4) & $\mathrm{Cs} 3-\mathrm{O}^{\mathrm{ix}}$ & $3.857(5)$ \\
\hline $\mathrm{Cs} 1-\mathrm{O}^{\mathrm{v}}$ & $3.570(5)$ & $\mathrm{Cs} 3-\mathrm{O}^{\mathrm{x}}$ & $3.942(4)$ \\
\hline $\mathrm{Cs} 1-\mathrm{O}^{\mathrm{iii}}$ & $4.044(4)$ & $\mathrm{Cs} 3-\mathrm{O} 7$ & $4.030(4)$ \\
\hline $\mathrm{Cs} 1-\mathrm{O} 1^{\mathrm{i}}$ & $4.050(4)$ & $\mathrm{Lu} 1-\mathrm{O}^{\mathrm{xi}}$ & $2.203(4)$ \\
\hline Cs1-O6 & $4.084(4)$ & $\mathrm{Lu} 1-\mathrm{O} 2$ & $2.210(4)$ \\
\hline $\mathrm{Cs} 1-\mathrm{O}^{\mathrm{iii}}$ & $4.092(4)$ & $\mathrm{Lu} 1-\mathrm{O} 9^{\mathrm{vi}}$ & $2.212(4)$ \\
\hline $\mathrm{Cs} 1-\mathrm{O} 7^{\mathrm{iii}}$ & $4.156(4)$ & $\mathrm{Lu} 1-\mathrm{O} 4$ & $2.236(4)$ \\
\hline $\mathrm{Cs} 1-\mathrm{O6}^{\mathrm{vi}}$ & $4.354(4)$ & $\mathrm{Lu} 1-\mathrm{O} 5$ & $2.247(4)$ \\
\hline $\mathrm{Cs} 2-\mathrm{O} 2^{\mathrm{ii}}$ & $2.980(4)$ & Lu1-O1 & $2.255(4)$ \\
\hline $\mathrm{Cs} 2-\mathrm{O}^{\mathrm{iii}}$ & 3.095 (4) & Si1-O5 & $1.606(4)$ \\
\hline $\mathrm{Cs} 2-\mathrm{O} 6$ & $3.130(4)$ & Si1-O9 & $1.609(4)$ \\
\hline $\mathrm{Cs} 2-\mathrm{O} 8$ & $3.143(4)$ & $\mathrm{Si} 1-\mathrm{O} 7$ & $1.661(4)$ \\
\hline $\mathrm{Cs} 2-\mathrm{O}^{\mathrm{iii}}$ & $3.170(5)$ & $\mathrm{Si} 1-\mathrm{O}^{\mathrm{vii}}$ & $1.662(4)$ \\
\hline $\mathrm{Cs} 2-\mathrm{O} 7$ & $3.221(4)$ & $\mathrm{Si} 2-\mathrm{O} 4^{\mathrm{iii}}$ & $1.599(4)$ \\
\hline $\mathrm{Cs} 2-\mathrm{O}^{\mathrm{vii}}$ & $3.752(5)$ & $\mathrm{Si} 2-\mathrm{O} 2^{\mathrm{iv}}$ & $1.605(4)$ \\
\hline $\mathrm{Cs} 2-\mathrm{O} 1$ & $3.824(4)$ & $\mathrm{Si} 2-\mathrm{O} 6$ & $1.650(4)$ \\
\hline $\mathrm{Cs} 2-\mathrm{O} 4^{\mathrm{iii}}$ & $3.926(4)$ & $\mathrm{Si} 2-\mathrm{O} 8$ & $1.659(4)$ \\
\hline $\mathrm{Cs} 2-\mathrm{O} 4^{\mathrm{i}}$ & $4.106(4)$ & $\mathrm{Si} 3-\mathrm{O}^{\text {vii }}$ & $1.594(4)$ \\
\hline $\mathrm{Cs} 2-\mathrm{O}^{\mathrm{vi}}$ & $4.224(4)$ & $\mathrm{Si} 3-\mathrm{O} 1^{\mathrm{xii}}$ & $1.608(4)$ \\
\hline $\mathrm{Cs} 2-\mathrm{O} 9^{\mathrm{iv}}$ & $4.228(4)$ & $\mathrm{Si} 3-\mathrm{O} 7$ & $1.655(4)$ \\
\hline $\mathrm{Cs} 3-\mathrm{O} 1$ & $2.936(4)$ & $\mathrm{Si} 3-\mathrm{O} 8$ & $1.664(4)$ \\
\hline $\mathrm{Cs} 3-\mathrm{O}^{\mathrm{iv}}$ & $2.983(4)$ & & \\
\hline
\end{tabular}

Symmetry codes: (i) $-x,-y+1, z+\frac{1}{2}$; (ii) $-x,-y, z+\frac{1}{2}$; (iii) $-x+\frac{1}{2}, y-\frac{1}{2}, z+\frac{1}{2}$; (iv) $-x+\frac{1}{2}, y+\frac{1}{2}, z+\frac{1}{2}$; (v) $x, y, z+1$; (vi) $x-\frac{1}{2},-y+\frac{1}{2}, z$; (vii) $-x+\frac{1}{2}, y-\frac{1}{2}, z-\frac{1}{2}$; (viii) $-x,-y+1, z-\frac{1}{2} ; \quad$ (ix) $-x+\frac{1}{2}, y+\frac{1}{2}, z-\frac{1}{2} ; \quad$ (x) $x, y+1, z ; \quad$ (xi) $x, y, z-1$; (xii) $x+\frac{1}{2},-y+\frac{1}{2}, z$.

The three distinct Cs sites, Cs1, Cs2 and Cs3, are situated in the voids of the three-dimensional framework composed of the silicate single chains connected by the $\mathrm{Lu}$ atoms. The Cs1 and $\mathrm{Cs} 2$ ions are surrounded by twelve $\mathrm{O}$ atoms, while the $\mathrm{Cs} 3$ ion is surrounded by ten O atoms. The BVS values for Cs1,

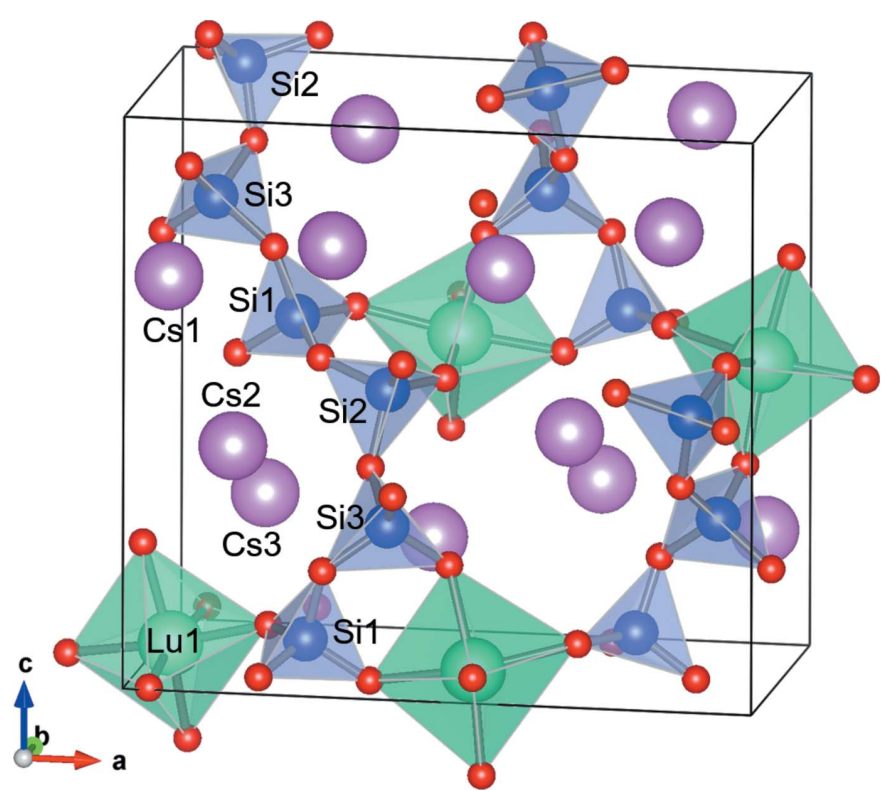

Figure 2

A polyhedral representation of $\mathrm{Cs}_{3} \mathrm{LuSi}_{3} \mathrm{O}_{9}$ showing the Lu1-centered oxygen octahedra (green) and Si-centered oxygen tetrahedra (blue) linked together to form a 3-D framework with Cs ions (purple) contained in the framework voids. 
Cs2, and Cs3 are 1.084, 0.919, 0.975, respectively [taking CsO distances in the range 2.869 (4)-4.228 (4) $\AA$ ], which are close to the expected value of 1 for the $\mathrm{Cs}^{\mathrm{I}}$ valence. All Cs sites are fully occupied, in contrast to the situation in the isotypic crystal structures of $\mathrm{Cs}_{3} R E^{\mathrm{III}} \mathrm{Ge}_{3} \mathrm{O}_{9}$ (Morrison et al., 2019). When $R E=\mathrm{Pr}, \mathrm{Nd}$ and $\mathrm{Sm}$, disorder is observed in all three Cs sites and for the $\mathrm{Eu}-\mathrm{Yb}$ analogues, disorder is observed in only two of the Cs sites.

The Madelung energy per formula unit for $\mathrm{Cs}_{3} \mathrm{LuSi}_{3} \mathrm{O}_{9}$, calculated using the structure parameters determined here within the VESTA program (Momma \& Izumi, 2011), is $-57,100 \mathrm{~kJ} \mathrm{~mol}^{-1}$, which is close to the value of $-56,700 \mathrm{~kJ} \mathrm{~mol}^{-1}$ (difference $\Delta=0.6 \%$ ) calculated from the equation: $3 / 2 \mathrm{Cs}_{2} \mathrm{O}+1 / 2 \mathrm{Lu}_{2} \mathrm{O}_{3}+3 \mathrm{SiO}_{2}=\mathrm{Cs}_{3} \mathrm{LuSi}_{3} \mathrm{O}_{9}$ using enthalpy values within VESTA of $-2,200,-15,600$ and $-15,200 \mathrm{~kJ} \mathrm{~mol}^{-1}$ for $\mathrm{Cs}_{2} \mathrm{O}, \mathrm{Lu}_{2} \mathrm{O}_{3}$ and $\mathrm{SiO}_{2}$, respectively, together with crystal-structure data for $\mathrm{Cs}_{2} \mathrm{O}$ (Tsai et al., 1956), $\mathrm{Lu}_{2} \mathrm{O}_{3}$ (Saiki et al., 1985) and $\mathrm{SiO}_{2}$ (d'Amour et al., 1979). The Madelung potentials for Cs1-Cs3 $(-8.7$ to $-9.2 \mathrm{~V})$, Lu1 $(-32.8 \mathrm{~V})$, and $\mathrm{Si} 1-\mathrm{Si} 3(-48.0 \mathrm{~V})$ in $\mathrm{Cs}_{3} \mathrm{LuSi}_{3} \mathrm{O}_{9}$ are in good agreement with those obtained in VESTA for Cs $(-9.0 \mathrm{~V})$ in $\mathrm{Cs}_{2} \mathrm{O}, \mathrm{Lu}(-31.3,-31.6 \mathrm{~V})$ in $\mathrm{Lu}_{2} \mathrm{O}$, and $\mathrm{Si}(-48.1 \mathrm{~V})$ in $\mathrm{SiO}_{2}$.

\section{Synthesis and crystallization}

Powders of $\mathrm{Cs}_{2} \mathrm{CO}_{3}$ (99.9\%, Kojundo Chemical Lab. Co., Ltd.), $\mathrm{Lu}_{2} \mathrm{O}_{3}$ (99.999\%, Nippon Yttrium Co., Ltd.) and $\mathrm{SiO}_{2}$ (99.999\%, Mitsuwa Chemicals Co., Ltd.) were used as starting materials. The $\mathrm{Lu}_{2} \mathrm{O}_{3}$ and $\mathrm{SiO}_{2}$ powders were heated at $1273 \mathrm{~K}$ for $10 \mathrm{~h}$ in air, and stored in an air oven heated at $453 \mathrm{~K}$, while the $\mathrm{Cs}_{2} \mathrm{CO}_{3}$ powder was heated at $673 \mathrm{~K}$ for $10 \mathrm{~h}$ and stored in an Ar-gas-filled glove box prior to use. $0.1955 \mathrm{~g}$ of $\mathrm{Cs}_{2} \mathrm{CO}_{3}$ powder were weighed in the glove box, and mixed with $0.0796 \mathrm{~g}$ of $\mathrm{Lu}_{2} \mathrm{O}_{3}$ and $0.00120 \mathrm{~g}$ of $\mathrm{SiO}_{2}$ (Cs:Lu:Si molar ratio $=3: 1: 0.5)$ in an agate mortar and pestle in air, and then pressed into a $5 \mathrm{~mm}$ diameter pellet. The pellet was heated at $1273 \mathrm{~K}$ for $3 \mathrm{~h}$ on a Pt plate in air, and cooled in the furnace by shutting off the power to the heater. The shape of the pellet was maintained; however, part of the sample had melted and solidified around the pellet on the Pt plate. Colourless transparent prismatic single crystals of around $0.1 \mathrm{~mm}$ in length were obtained from the melted part of the pellet. The unmelted part of the pellet consisted of $\mathrm{Lu}_{2} \mathrm{O}_{3}$ and $\mathrm{Cs}_{3} \mathrm{LuSi}_{3} \mathrm{O}_{9}$, which was verified by powder X-ray diffraction using a Bruker AXS, D2 PHASER diffractometer with $\mathrm{Cu} \mathrm{K \alpha}$ radiation.

\section{Refinement}

Crystal data, data collection and structure refinement details are summarized in Table 2. Atomic coordinates and site labels were standardized with Structure Tidy (Gelato \& Parthé, 1987) implemented in PLATON (Spek, 2020). The crystal structure was refined with consideration of twinning by inversion, which revealed a minor contribution of $3.1(8) \%$ for the inversionrelated twin component.
Table 2

Experimental details.

\begin{tabular}{|c|c|}
\hline \multicolumn{2}{|l|}{ Crystal data } \\
\hline Chemical formula & $\mathrm{Cs}_{3} \mathrm{LuSi}_{3} \mathrm{O}_{9}$ \\
\hline$M_{\mathrm{r}}$ & 801.97 \\
\hline Crystal system, space group & Orthorhombic, Pna $_{1}$ \\
\hline Temperature (K) & 300 \\
\hline$a, b, c(\AA)$ & $13.3322(3), 6.8618(2), 12.2313(3)$ \\
\hline$V\left(\AA^{3}\right)$ & $1118.95(5)$ \\
\hline$Z$ & 4 \\
\hline Radiation type & Mo $K \alpha$ \\
\hline$\mu\left(\mathrm{mm}^{-1}\right)$ & 18.79 \\
\hline Crystal size $(\mathrm{mm})$ & $0.09 \times 0.05 \times 0.03$ \\
\hline \multicolumn{2}{|l|}{ Data collection } \\
\hline Diffractometer & Bruker APEXII CCD \\
\hline Absorption correction & $\begin{array}{l}\text { Multi-scan (SADABS; Bruker, } \\
\text { 2018) }\end{array}$ \\
\hline$T_{\min }, T_{\max }$ & $0.39,0.58$ \\
\hline $\begin{array}{l}\text { No. of measured, independent and } \\
\text { observed }[I>2 \sigma(I)] \text { reflections }\end{array}$ & $24311,3094,3085$ \\
\hline$R_{\text {int }}$ & 0.034 \\
\hline$(\sin \theta / \lambda)_{\max }\left(\AA^{-1}\right)$ & 0.695 \\
\hline \multicolumn{2}{|l|}{ Refinement } \\
\hline$R\left[F^{2}>2 \sigma\left(F^{2}\right)\right], w R\left(F^{2}\right), S$ & $0.014,0.036,1.11$ \\
\hline No. of reflections & 3094 \\
\hline No. of parameters & 147 \\
\hline No. of restraints & 1 \\
\hline$\Delta \rho_{\max }, \Delta \rho_{\min }\left(\mathrm{e} \AA^{-3}\right)$ & $1.87,-1.18$ \\
\hline Absolute structure & Refined as an inversion twin \\
\hline Absolute structure parameter & $0.031(8)$ \\
\hline
\end{tabular}

Computer programs: BIS, APEX3 and SAINT (Bruker, 2018), SHELXT2014/5 (Sheldrick, 2015a), SHELXL2014/7 (Sheldrick, 2015b), VESTA (Momma \& Izumi, 2011) and publCIF (Westrip, 2010).

\section{Acknowledgements}

The authors thank Eiko Kobayashi and Yuko Suzuki for their assistance with the sample preparation.

\section{Funding information}

This work was supported in part by Mitsubishi Chemical Corporation (a joint research project of Tohoku University and Mitsubishi Chemical Corporation, J190002825).

\section{References}

Amour, H. d', Denner, W. \& Schulz, H. (1979). Acta Cryst. B35, 550555.

Ananias, D., Carlos, L. D. \& Rocha, J. (2006). Opt. Mater. 28, 582-586.

Ananias, D., Rainho, J. P., Ferreira, A., Lopes, M., Morais, C. M., Rocha, J. \& Carlos, L. D. (2002). Chem. Mater. 14, 1767-1772.

Arlt, T. \& Angel, R. J. (2000). Phys. Chem. Miner. 27, 719-731.

Banks, E. \& Kim, C. H. (1985). J. Electrochem. Soc. 132, 2617-2621.

Bruker (2018). BIS, $A P E X 3, S A I N T$ and $S A D A B S$. Bruker AXS inc., Madison, Wisconsin, USA.

Dorenbos, P., van Eijk, C. W. E., Bos, A. J. J. \& Melcher, C. L. (1994). J. Lumin. 60-61, 979-982.

Filipenko, O. S., Ponomarev, V. I., Dimitrova, O.V. \& Atovmyan, L. O. (1988). Kristallografiya, 33, 1122-1127.

Gagné, O. C. \& Hawthorne, F. C. (2015). Acta Cryst. B71, 562-578.

Gelato, L. M. \& Parthé, E. (1987). J. Appl. Cryst. 20, 139-143.

Kahlenberg, V., Mörtl, A. \& Krüger, H. (2015). Z. Kristallogr. 230, 87-95.

Kim, C. H., Qiu, B. \& Banks, E. (1985). J. Electrochem. Soc. 132, 1340-1345. 
Kostova, M. H., Ananias, D., Paz, F. A. A., Ferreira, A., Rocha, J. \& Carlos, L. D. (2007). J. Phys. Chem. B, 111, 3576-3582.

Liebau, F. (1985). Structural Chemistry of Silicates. Berlin: SpringerVerlag.

Maksimov, B. A., Kalinin, V. R., Merinov, B. V., Ilyukhin, V. V. \& Belov, N. V. (1980). Dokl. Akad. Nauk SSSR, 252, 875-879.

Melcher, C. L. \& Schweitzer, J. S. (1992). IEEE Trans. Nucl. Sci. 39, 502-505.

Momma, K. \& Izumi, F. (2011). J. Appl. Cryst. 44, 1272-1276.

Morrison, G., Spagnuolo, N. R., Karakalos, S. G. \& Zur Loye, H.-C. (2019). Inorg. Chem. 58, 8702-8709.

Ponomarev, V. I., Filipenko, O. S. \& Atovmyan, L. O. (1988). Kristallografiya, 33, 98-104.

Redhammer, G. J. \& Roth, G. (2004). Z. Krist. Cryst. Mater. 219, 585605.

Saiki, A., Ishizawa, N., Mizutani, N. \& Kato, M. (1985). Yogyo Kyokaishi 93, 649-654.

Schäfer, M. C., Zitzer, S. \& Schleid, T. (2012). Z. Kristallogr. 227, 476482.
Shannon, R. D., Gier, T. E., Foris, C. M., Nelen, J. A. \& Appelman, D. E. (1980). Phys. Chem. Miner. 5, 245-253.

Sheldrick, G. M. (2015a). Acta Cryst. A71, 3-8.

Sheldrick, G. M. (2015b). Acta Cryst. C71, 3-8.

Simura, R. \& Yamane, H. (2020a). Acta Cryst. E76, 752-755.

Simura, R. \& Yamane, H. (2020b). Acta Cryst. E76, 1708-1711.

Spek, A. L. (2020). Acta Cryst. E76, 1-11.

Többens, D. M., Kahlenberg, V. \& Kaindl, R. (2005). Inorg. Chem. 44, 9554-9560.

Tsai, K.-R., Harris, P. M. \& Lassettre, E. N. (1956). J. Phys. Chem. 60, 338-344.

Westrip, S. P. (2010). J. Appl. Cryst. 43, 920-925.

Wierzbicka-Wieczorek, M., Kolitsch, U., Nasdala, L. \& Tillmanns, E. (2010). Miner. Mag. 74, 979-990.

Zhang, Z., Wang, Y. \& Zhang, J. (2008b). Mater. Lett. 62, 846848.

Zhang, Z., Wang, Y., Zhang, J. \& Hao, Y. (2008a). Mater. Res. Bull. 43, 926-931. 


\section{supporting information}

Acta Cryst. (2021). E77, 1239-1242［https://doi.org/10.1107/S2056989021011439]

\section{Crystal structure of chain silicate $\mathrm{Cs}_{3} \mathrm{LuSi}_{3} \mathrm{O}_{9}$}

\section{Hiromitsu Kimura and Hisanori Yamane}

\section{Computing details}

Data collection: BIS (Bruker, 2018); cell refinement: APEX3 (Bruker, 2018); data reduction: SAINT (Bruker, 2018); program(s) used to solve structure: SHELXT2014/5 (Sheldrick, 2015a); program(s) used to refine structure:

SHELXL2014/7 (Sheldrick, 2015b); molecular graphics: VESTA (Momma \& Izumi, 2011); software used to prepare material for publication: publCIF (Westrip, 2010).

Tricaesium lutetium trisilicate

Crystal data

$\mathrm{Cs}_{3} \mathrm{LuSi}_{3} \mathrm{O}_{9}$

$M_{r}=801.97$

Orthorhombic, Pna2 1

$a=13.3322(3) \AA$

$b=6.8618(2) \AA$

$c=12.2313(3) \AA$

$V=1118.95(5) \AA^{3}$

$Z=4$

$F(000)=1400$

\section{Data collection}

Bruker APEXII CCD diffractometer

Radiation source: micro focus sealed tube Detector resolution: 7.3910 pixels $\mathrm{mm}^{-1}$ $\varphi$ and $\omega$ scans

Absorption correction: multi-scan

(SADABS; Bruker, 2018)

$T_{\min }=0.39, T_{\max }=0.58$

Refinement

Refinement on $F^{2}$

Least-squares matrix: full

$R\left[F^{2}>2 \sigma\left(F^{2}\right)\right]=0.014$

$w R\left(F^{2}\right)=0.036$

$S=1.11$

3094 reflections

147 parameters

1 restraint
$D_{\mathrm{x}}=4.761 \mathrm{Mg} \mathrm{m}^{-3}$

Mo $K \alpha$ radiation, $\lambda=0.71073 \AA$

Cell parameters from 351 reflections

$\theta=3.1-31.4^{\circ}$

$\mu=18.79 \mathrm{~mm}^{-1}$

$T=300 \mathrm{~K}$

Prismatic, translucent colourless

$0.09 \times 0.05 \times 0.03 \mathrm{~mm}$

24311 measured reflections 3094 independent reflections 3085 reflections with $I>2 \sigma(I)$

$R_{\text {int }}=0.034$

$\theta_{\max }=29.6^{\circ}, \theta_{\min }=3.1^{\circ}$

$h=-18 \rightarrow 18$

$k=-9 \rightarrow 9$

$l=-16 \rightarrow 16$

$w=1 /\left[\sigma^{2}\left(F_{\mathrm{o}}^{2}\right)+2.7745 P\right]$

where $P=\left(F_{\mathrm{o}}^{2}+2 F_{\mathrm{c}}^{2}\right) / 3$

$(\Delta / \sigma)_{\max }<0.001$

$\Delta \rho_{\max }=1.87 \mathrm{e} \AA^{-3}$

$\Delta \rho_{\min }=-1.18$ e $\AA^{-3}$

Extinction correction: SHELXL2017/1

(Sheldrick 2015b)

Extinction coefficient: 0.00160 (14)

Absolute structure: Refined as an inversion twin

Absolute structure parameter: 0.031 (8) 


\section{Special details}

Geometry. All esds (except the esd in the dihedral angle between two 1.s. planes) are estimated using the full covariance matrix. The cell esds are taken into account individually in the estimation of esds in distances, angles and torsion angles; correlations between esds in cell parameters are only used when they are defined by crystal symmetry. An approximate (isotropic) treatment of cell esds is used for estimating esds involving l.s. planes.

Refinement. Refined as a two-component inversion twin.

Fractional atomic coordinates and isotropic or equivalent isotropic displacement parameters $\left(\AA^{2}\right)$

\begin{tabular}{lllll}
\hline & $x$ & $y$ & $z$ & $U_{\text {iss }} / U_{\text {eq }}$ \\
\hline Cs1 & $0.06219(4)$ & $0.10542(6)$ & $0.71151(4)$ & $0.02535(10)$ \\
Cs2 & $0.16894(3)$ & $0.05816(7)$ & $0.42547(4)$ & $0.02637(11)$ \\
Cs3 & $0.17391(3)$ & $0.55846(6)$ & $0.28251(3)$ & $0.02153(9)$ \\
Lu1 & $0.04370(2)$ & $0.34863(3)$ & $0.03915(2)$ & $0.00583(6)$ \\
Si1 & $0.28028(11)$ & $0.1098(2)$ & $0.08655(12)$ & $0.0070(2)$ \\
Si2 & $0.38805(11)$ & $0.3492(2)$ & $0.49763(13)$ & $0.0064(2)$ \\
Si3 & $0.41034(10)$ & $0.1056(2)$ & $0.29080(12)$ & $0.0068(2)$ \\
O1 & $0.0020(3)$ & $0.3178(6)$ & $0.2170(3)$ & $0.0118(7)$ \\
O2 & $0.0386(3)$ & $0.0346(6)$ & $-0.0005(4)$ & $0.0113(7)$ \\
O3 & $0.0643(3)$ & $0.4213(6)$ & $0.8652(3)$ & $0.0144(8)$ \\
O4 & $0.0722(3)$ & $0.6662(6)$ & $0.0659(3)$ & $0.0122(8)$ \\
O5 & $0.2041(3)$ & $0.2910(6)$ & $0.0854(4)$ & $0.0133(8)$ \\
O6 & $0.2752(3)$ & $0.4080(5)$ & $0.5419(4)$ & $0.0138(7)$ \\
O7 & $0.3124(3)$ & $0.0510(6)$ & $0.2136(3)$ & $0.0123(7)$ \\
O8 & $0.3669(3)$ & $0.2855(6)$ & $0.3688(3)$ & $0.0099(7)$ \\
O9 & $0.3794(3)$ & $0.1463(6)$ & $0.0142(3)$ & $0.0137(9)$ \\
& & & &
\end{tabular}

Atomic displacement parameters $\left(\AA^{2}\right)$

\begin{tabular}{lllllll}
\hline & $U^{11}$ & $U^{22}$ & $U^{33}$ & $U^{12}$ & $U^{13}$ & $U^{23}$ \\
\hline Cs1 & $0.0370(2)$ & $0.01343(17)$ & $0.0257(2)$ & $0.00434(16)$ & $-0.01766(18)$ & $-0.00375(16)$ \\
Cs2 & $0.02074(19)$ & $0.0252(2)$ & $0.0331(2)$ & $-0.00933(15)$ & $0.00863(16)$ & $-0.00168(17)$ \\
Cs3 & $0.01763(17)$ & $0.0304(2)$ & $0.01654(17)$ & $0.00004(14)$ & $-0.00250(13)$ & $-0.00726(16)$ \\
Lu1 & $0.00583(9)$ & $0.00579(9)$ & $0.00586(9)$ & $-0.00008(6)$ & $0.00064(8)$ & $0.00022(8)$ \\
Si1 & $0.0049(6)$ & $0.0097(6)$ & $0.0063(6)$ & $-0.0004(5)$ & $-0.0006(5)$ & $-0.0016(5)$ \\
Si2 & $0.0071(6)$ & $0.0052(6)$ & $0.0069(5)$ & $0.0001(4)$ & $0.0003(5)$ & $-0.0005(5)$ \\
Si3 & $0.0076(6)$ & $0.0080(6)$ & $0.0047(6)$ & $0.0004(5)$ & $-0.0004(5)$ & $-0.0005(5)$ \\
O1 & $0.0123(17)$ & $0.0161(17)$ & $0.0070(16)$ & $0.0002(15)$ & $0.0029(15)$ & $0.0011(15)$ \\
O2 & $0.0087(16)$ & $0.0064(16)$ & $0.0189(18)$ & $0.0019(13)$ & $0.0002(15)$ & $-0.0011(15)$ \\
O3 & $0.023(2)$ & $0.0113(17)$ & $0.0088(18)$ & $-0.0047(16)$ & $0.0032(16)$ & $0.0011(15)$ \\
O4 & $0.020(2)$ & $0.0069(17)$ & $0.0092(18)$ & $0.0002(14)$ & $0.0028(14)$ & $0.0005(13)$ \\
O5 & $0.0085(17)$ & $0.0122(17)$ & $0.019(2)$ & $0.0029(14)$ & $-0.0036(14)$ & $-0.0031(16)$ \\
O6 & $0.0117(16)$ & $0.0112(15)$ & $0.0186(18)$ & $-0.0005(13)$ & $0.0073(18)$ & $-0.007(2)$ \\
O7 & $0.0124(17)$ & $0.0167(19)$ & $0.0079(17)$ & $-0.0043(14)$ & $-0.0009(14)$ & $0.0010(16)$ \\
O8 & $0.0121(16)$ & $0.0098(16)$ & $0.0077(16)$ & $0.0056(14)$ & $-0.0002(14)$ & $-0.0013(14)$ \\
O9 & $0.0071(16)$ & $0.026(2)$ & $0.008(2)$ & $-0.0012(15)$ & $0.0023(12)$ & $0.0015(14)$ \\
& & & & & & \\
\hline
\end{tabular}


Geometric parameters $\left(\AA,{ }^{\circ}\right)$

\begin{tabular}{|c|c|c|c|}
\hline $\mathrm{Cs} 1-\mathrm{O} 3$ & $2.869(4)$ & $\mathrm{Cs} 2-\mathrm{Si} 1^{\mathrm{iv}}$ & $4.3209(16)$ \\
\hline $\mathrm{Cs} 1-\mathrm{O} 4^{\mathrm{i}}$ & $2.972(4)$ & Cs2-Lu1 ${ }^{\mathrm{iii}}$ & $4.3218(5)$ \\
\hline $\mathrm{Cs} 1-\mathrm{O} 1^{\mathrm{ii}}$ & $3.028(4)$ & $\mathrm{Cs} 2-\mathrm{Si} 1$ & $4.4174(15)$ \\
\hline $\mathrm{Cs} 1-\mathrm{O} 8^{\mathrm{iii}}$ & 3.069 (4) & $\mathrm{Cs} 2-\mathrm{Si}^{\mathrm{v}}$ & $4.4636(15)$ \\
\hline $\mathrm{Cs} 1-\mathrm{O} 2^{\mathrm{ii}}$ & $3.074(5)$ & $\mathrm{Cs} 3-\mathrm{O} 1$ & $2.936(4)$ \\
\hline $\mathrm{Cs} 1-\mathrm{O}^{\mathrm{iv}}$ & 3.485 (4) & $\mathrm{Cs} 3-\mathrm{O}^{\mathrm{iv}}$ & $2.983(4)$ \\
\hline $\mathrm{Cs} 1-\mathrm{Si} 2^{\mathrm{v}}$ & $3.5115(16)$ & $\mathrm{Cs} 3-\mathrm{O} 5$ & $3.057(4)$ \\
\hline $\mathrm{Cs} 1-\mathrm{O} 2^{\mathrm{vi}}$ & $3.570(5)$ & $\mathrm{Cs} 3-\mathrm{O} 4$ & $3.066(4)$ \\
\hline $\mathrm{Cs} 1-\mathrm{Si} 3^{\mathrm{iii}}$ & $3.5829(15)$ & $\mathrm{Cs} 3-\mathrm{O} 3^{\mathrm{ix}}$ & $3.335(5)$ \\
\hline $\mathrm{Cs} 1-\mathrm{Si}^{\mathrm{iv}}$ & $3.5852(15)$ & $\mathrm{Cs} 3-\mathrm{O} 8$ & $3.354(4)$ \\
\hline $\mathrm{Cs} 1-\mathrm{Cs} 3^{\mathrm{iii}}$ & $3.6383(6)$ & $\mathrm{Cs} 3-\mathrm{O} 6$ & $3.599(5)$ \\
\hline $\mathrm{Cs} 1-\mathrm{Cs} 2$ & $3.7909(7)$ & $\mathrm{Cs} 3-\mathrm{Si}^{\mathrm{v}}$ & $3.6912(14)$ \\
\hline $\mathrm{Cs} 1-\mathrm{Si} 2^{\mathrm{iii}}$ & $3.9722(16)$ & $\mathrm{Cs} 3-\mathrm{Lu} 1$ & $3.7345(5)$ \\
\hline $\mathrm{Cs} 1-\mathrm{Cs} 3^{\mathrm{i}}$ & $3.9977(6)$ & $\mathrm{Cs} 3-\mathrm{Si} 1^{\mathrm{iv}}$ & $3.7851(15)$ \\
\hline $\mathrm{Cs} 1-\mathrm{Lu} 1^{\mathrm{ii}}$ & $4.0179(5)$ & $\mathrm{Cs} 3-06^{\mathrm{x}}$ & $3.857(5)$ \\
\hline $\mathrm{Cs} 1-\mathrm{O} 9^{\mathrm{iii}}$ & $4.044(4)$ & $\mathrm{Cs} 3-\mathrm{O} 7^{\mathrm{xi}}$ & $3.942(4)$ \\
\hline $\mathrm{Cs} 1-\mathrm{O} 1^{\mathrm{i}}$ & $4.050(4)$ & $\mathrm{Cs} 3-\mathrm{O} 7$ & $4.030(4)$ \\
\hline $\mathrm{Cs} 1-\mathrm{O} 6$ & $4.084(4)$ & $\mathrm{Cs} 3-\mathrm{Si} 2^{\mathrm{x}}$ & $4.0992(16)$ \\
\hline $\mathrm{Cs} 1-\mathrm{O} 5^{\mathrm{iii}}$ & $4.092(4)$ & $\mathrm{Cs} 3-\mathrm{Si} 2$ & $4.1396(15)$ \\
\hline $\mathrm{Cs} 1-\mathrm{O} 7^{\mathrm{iii}}$ & $4.156(4)$ & $\mathrm{Cs} 3-\mathrm{Si} 1$ & $4.1512(15)$ \\
\hline $\mathrm{Cs} 1-\mathrm{Cs} 2^{\mathrm{ii}}$ & $4.1958(7)$ & $\mathrm{Cs} 3-\mathrm{Lu} 1^{\mathrm{i}}$ & $4.3217(5)$ \\
\hline $\mathrm{Cs} 1-\mathrm{Si} 1^{\mathrm{iii}}$ & $4.2791(15)$ & $\mathrm{Cs} 3-\mathrm{O}^{\mathrm{iv}}$ & $4.349(4)$ \\
\hline $\mathrm{Cs} 1-\mathrm{Si} 1^{\mathrm{iv}}$ & $4.3275(16)$ & $\mathrm{Cs} 3-\mathrm{O} 3^{\mathrm{x}}$ & $4.405(5)$ \\
\hline $\mathrm{Cs} 1-\mathrm{Lu} 1^{\mathrm{vi}}$ & $4.3481(5)$ & $\mathrm{Cs} 3-\mathrm{Si} 3$ & $4.4276(15)$ \\
\hline $\mathrm{Cs} 1-\mathrm{O}^{\mathrm{v}}$ & $4.354(4)$ & $\mathrm{Cs} 3-\mathrm{O} 2^{\mathrm{iv}}$ & $4.665(4)$ \\
\hline $\mathrm{Cs} 2-\mathrm{O} 2^{\mathrm{ii}}$ & $2.980(4)$ & $\mathrm{Lu} 1-\mathrm{O} 3^{\mathrm{xii}}$ & $2.203(4)$ \\
\hline $\mathrm{Cs} 2-\mathrm{O} 9^{\mathrm{iii}}$ & 3.095 (4) & $\mathrm{Lu} 1-\mathrm{O} 2$ & $2.210(4)$ \\
\hline $\mathrm{Cs} 2-\mathrm{O} 6$ & $3.130(4)$ & $\mathrm{Lu} 1-\mathrm{O}^{\mathrm{v}}$ & $2.212(4)$ \\
\hline $\mathrm{Cs} 2-\mathrm{O} 8$ & $3.143(4)$ & $\mathrm{Lu} 1-\mathrm{O} 4$ & $2.236(4)$ \\
\hline $\mathrm{Cs} 2-\mathrm{O} 5^{\mathrm{iii}}$ & $3.170(5)$ & Lu1-O5 & $2.247(4)$ \\
\hline $\mathrm{Cs} 2-\mathrm{O} 7$ & $3.221(4)$ & $\mathrm{Lu} 1-\mathrm{O} 1$ & $2.255(4)$ \\
\hline $\mathrm{Cs} 2-\mathrm{Si} 3$ & $3.6301(15)$ & $\mathrm{Si} 1-\mathrm{O} 5$ & $1.606(4)$ \\
\hline $\mathrm{Cs} 2-\mathrm{Si} 2$ & $3.6470(15)$ & Si1-O9 & $1.609(4)$ \\
\hline $\mathrm{Cs} 2-\mathrm{Si} 1^{\mathrm{iii}}$ & $3.7153(16)$ & $\mathrm{Si1}-\mathrm{O} 7$ & $1.661(4)$ \\
\hline $\mathrm{Cs} 2-\mathrm{O} 3^{\mathrm{vii}}$ & $3.752(5)$ & $\mathrm{Si} 1-\mathrm{O} 6^{\mathrm{vii}}$ & $1.662(4)$ \\
\hline $\mathrm{Cs} 2-\mathrm{O} 1$ & $3.824(4)$ & $\mathrm{Si} 2-\mathrm{O} 4^{\mathrm{iii}}$ & $1.599(4)$ \\
\hline $\mathrm{Cs} 2-\mathrm{Cs} 3^{\mathrm{viii}}$ & $3.8495(6)$ & $\mathrm{Si} 2-\mathrm{O} 2^{\mathrm{iv}}$ & 1.605 (4) \\
\hline $\mathrm{Cs} 2-\mathrm{Cs} 3$ & $3.8532(7)$ & $\mathrm{Si} 2-\mathrm{O} 6$ & $1.650(4)$ \\
\hline $\mathrm{Cs} 2-\mathrm{Si} 2^{\mathrm{v}}$ & $3.8996(15)$ & $\mathrm{Si} 2-\mathrm{O} 8$ & $1.659(4)$ \\
\hline $\mathrm{Cs} 2-\mathrm{O} 4^{\mathrm{iii}}$ & $3.926(4)$ & $\mathrm{Si} 3-\mathrm{O} 3^{\mathrm{vii}}$ & 1.594 (4) \\
\hline $\mathrm{Cs} 2-\mathrm{O} 4^{\mathrm{i}}$ & $4.106(4)$ & $\mathrm{Si} 3-\mathrm{O} 1^{\mathrm{xiii}}$ & $1.608(4)$ \\
\hline $\mathrm{Cs} 2-\mathrm{Lu} 1^{\mathrm{ii}}$ & $4.2145(5)$ & $\mathrm{Si} 3-\mathrm{O} 7$ & $1.655(4)$ \\
\hline $\mathrm{Cs} 2-\mathrm{O}^{\mathrm{v}}$ & $4.224(4)$ & $\mathrm{Si} 3-\mathrm{O} 8$ & $1.664(4)$ \\
\hline $\mathrm{Cs} 2-\mathrm{O} 9^{\mathrm{iv}}$ & $4.228(4)$ & & \\
\hline $\mathrm{O} 3-\mathrm{Cs} 1-\mathrm{O} 4^{\mathrm{i}}$ & $89.99(12)$ & $\mathrm{Cs} 2-\mathrm{Cs} 3-\mathrm{Lu} 1^{\mathrm{i}}$ & $77.894(10)$ \\
\hline
\end{tabular}




$$
\begin{aligned}
& \mathrm{O} 3-\mathrm{Cs} 1-\mathrm{O} 1^{\mathrm{ii}} \\
& \mathrm{O} 4-\mathrm{Cs} 1-\mathrm{O} 1^{\mathrm{ii}} \\
& \mathrm{O} 3-\mathrm{Cs} 1-\mathrm{O}^{\mathrm{iii}} \\
& \mathrm{O} 4-\mathrm{Cs} 1-\mathrm{O}^{\mathrm{iii}} \\
& \mathrm{O} 1^{1 i}-\mathrm{Cs} 1-\mathrm{O}^{\mathrm{iii}} \\
& \mathrm{O} 3-\mathrm{Cs} 1-\mathrm{O} 2^{\mathrm{ii}} \\
& \mathrm{O} 4^{\mathrm{i}}-\mathrm{Cs} 1-\mathrm{O}^{2 \mathrm{ii}} \\
& \mathrm{O} 1^{\mathrm{ii}}-\mathrm{Cs} 1-\mathrm{O} 2^{\mathrm{ii}}
\end{aligned}
$$

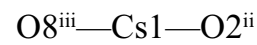

$$
\begin{aligned}
& \mathrm{O} 3-\mathrm{Cs} 1-\mathrm{O}^{\mathrm{iv}} \\
& \mathrm{O} 4-\mathrm{Cs} 1-\mathrm{O}^{\mathrm{i}}{ }^{\mathrm{iv}} \\
& \mathrm{O} 1^{i i}-\mathrm{Cs} 1-\mathrm{O}^{\mathrm{iv}} \\
& \mathrm{O} 8^{i i i}-\mathrm{Cs} 1-\mathrm{O}^{\mathrm{iv}} \\
& \mathrm{O} 2^{\mathrm{ii}}-\mathrm{Cs} 1-\mathrm{O}^{\mathrm{iv}} \\
& \mathrm{O} 3-\mathrm{Cs} 1-\mathrm{Si}^{\mathrm{v}}
\end{aligned}
$$



$$
\begin{aligned}
& \mathrm{O} 1^{\mathrm{ii}}-\mathrm{Cs} 1-\mathrm{Si}^{\mathrm{v}} \\
& \mathrm{O} 8^{\mathrm{iii}}-\mathrm{Cs} 1-\mathrm{Si}_{2}^{\mathrm{v}} \\
& \mathrm{O} 2^{\mathrm{ii}}-\mathrm{Cs} 1-\mathrm{Si}^{\mathrm{v}} \\
& \mathrm{O}^{7 \mathrm{iv}}-\mathrm{Cs} 1-\mathrm{Si}^{2}{ }^{\mathrm{v}} \\
& \mathrm{O} 3-\mathrm{Cs} 1-\mathrm{O}^{\mathrm{vi}} \\
& \mathrm{O} 4^{\mathrm{i}}-\mathrm{Cs} 1-\mathrm{O} 2^{\mathrm{vi}} \\
& \mathrm{O} 1^{\mathrm{ii}}-\mathrm{Cs} 1-\mathrm{O}^{\mathrm{vi}} \\
& \mathrm{O} 8^{\mathrm{iii}}-\mathrm{Cs} 1-\mathrm{O} 2^{\mathrm{vi}} \\
& \mathrm{O} 2^{\mathrm{ii}}-\mathrm{Cs} 1-\mathrm{O}^{\mathrm{vi}} \\
& \mathrm{O} 7^{\mathrm{iv}}-\mathrm{Cs} 1-\mathrm{O} 2^{\mathrm{vi}} \\
& \mathrm{Si} 2^{\mathrm{v}}-\mathrm{Cs} 1-\mathrm{O} 2^{\mathrm{vi}} \\
& \mathrm{O} 3-\mathrm{Cs} 1-\mathrm{Si}^{3 i i}
\end{aligned}
$$

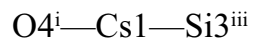

$$
\begin{aligned}
& \mathrm{O} 1^{\mathrm{ii}}-\mathrm{Cs} 1-\mathrm{Si}^{3}{ }^{\mathrm{iii}} \\
& \mathrm{O} 8^{\mathrm{iii}}-\mathrm{Cs} 1-\mathrm{Si}^{3}{ }^{\mathrm{iii}} \\
& \mathrm{O} 2^{\mathrm{ii}}-\mathrm{Cs} 1-\mathrm{Si}_{3}{ }^{\mathrm{iii}} \\
& \mathrm{O} 7^{\mathrm{iv}}-\mathrm{Cs} 1-\mathrm{Si}^{3}{ }^{\mathrm{iii}} \\
& \mathrm{Si} 2^{\mathrm{v}}-\mathrm{Cs} 1-\mathrm{Si}_{3}{ }^{\mathrm{iii}} \\
& \mathrm{O} 2^{\mathrm{vi}}-\mathrm{Cs} 1-\mathrm{Si}^{\mathrm{iii}} \\
& \mathrm{O} 3-\mathrm{Cs} 1-\mathrm{Si}^{3}{ }^{\text {iv }} \\
& \mathrm{O} 4-\mathrm{Cs} 1-\mathrm{Si}^{\mathrm{iv}} \\
& \mathrm{O} 1^{\mathrm{ii}}-\mathrm{Cs} 1-\mathrm{Si}^{\text {iv }} \\
& \mathrm{O}^{\mathrm{iii}}-\mathrm{Cs} 1-\mathrm{Si}^{\mathrm{iv}} \\
& \mathrm{O} 2^{\mathrm{ii}}-\mathrm{Cs} 1-\mathrm{Si}^{\mathrm{iv}} \\
& \mathrm{O}^{\mathrm{iv}}-\mathrm{Cs} 1-\mathrm{Si}^{3 \mathrm{iv}} \\
& \mathrm{Si}^{\mathrm{v}}-\mathrm{Cs} 1-\mathrm{Si}^{3 \mathrm{iv}} \\
& \mathrm{O} 2^{\text {vi }}-\mathrm{Cs} 1-\mathrm{Si}^{3 \mathrm{iv}} \\
& \mathrm{Si}^{3 i i}-\mathrm{Cs} 1-\mathrm{Si}^{3{ }^{\mathrm{iv}}} \\
& \mathrm{O} 3-\mathrm{Cs} 1-\mathrm{Cs}^{\mathrm{iii}} \\
& \mathrm{O} 4 \text { i-Cs } 1-\mathrm{Cs}^{\mathrm{iii}} \\
& \mathrm{O} 1^{\mathrm{ii}}-\mathrm{Cs} 1-\mathrm{Cs} 3^{\mathrm{iii}} \\
& \mathrm{O} 8^{\mathrm{iii}}-\mathrm{Cs} 1-\mathrm{Cs} 3^{\mathrm{iii}}
\end{aligned}
$$

$135.45(11)$

$110.40(11)$

$97.28(11)$

$159.80(11)$

$52.20(10)$

$142.44(12)$

$52.85(11)$

\begin{tabular}{|c|c|}
\hline $\mathrm{O} 6^{\mathrm{x}}-\mathrm{Cs} 3-\mathrm{Lu}^{\mathrm{i}}$ & $125.50(6)$ \\
\hline $\mathrm{O} 7^{\mathrm{xi}}-\mathrm{Cs} 3-\mathrm{Lu} 1^{\mathrm{i}}$ & $110.07(6)$ \\
\hline $\mathrm{Cs} 1^{\mathrm{ix}}-\mathrm{Cs} 3-\mathrm{Lu} 1^{\mathrm{i}}$ & $62.878(9)$ \\
\hline $\mathrm{O} 7-\mathrm{Cs} 3-\mathrm{Lu} 1^{\mathrm{i}}$ & $125.93(6)$ \\
\hline $\mathrm{Si} 2^{\mathrm{x}}-\mathrm{Cs} 3-\mathrm{Lu} 1^{\mathrm{i}}$ & $114.23(2)$ \\
\hline $\mathrm{Si} 2-\mathrm{Cs} 3-\mathrm{Lu} 1^{\mathrm{i}}$ & $93.01(2)$ \\
\hline $\mathrm{Si} 1-\mathrm{Cs} 3-\mathrm{Lu} 1^{\mathrm{i}}$ & $139.30(2)$ \\
\hline $\mathrm{O} 1-\mathrm{Cs} 3-\mathrm{O}^{\mathrm{iv}}$ & $136.94(10)$ \\
\hline $\mathrm{O} 9^{\mathrm{iv}}-\mathrm{Cs} 3-\mathrm{O}^{\mathrm{iv}}$ & $37.42(9)$ \\
\hline $\mathrm{O} 5-\mathrm{Cs} 3-\mathrm{O}^{\mathrm{iv}}$ & $147.45(6)$ \\
\hline $\mathrm{O} 4-\mathrm{Cs} 3-\mathrm{O}^{\mathrm{iv}}$ & $144.37(9)$ \\
\hline $\mathrm{O} 3^{\mathrm{ix}}-\mathrm{Cs} 3-\mathrm{O} 5^{\mathrm{iv}}$ & $94.74(9)$ \\
\hline $\mathrm{O} 8-\mathrm{Cs} 3-\mathrm{O}^{\mathrm{iv}}$ & $69.50(9)$ \\
\hline $\mathrm{O} 6-\mathrm{Cs} 3-\mathrm{O}^{\mathrm{iv}}$ & $38.20(8)$ \\
\hline $\mathrm{Cs} 1^{\mathrm{x}}-\mathrm{Cs} 3-\mathrm{O}^{\mathrm{iv}}$ & $79.00(5)$ \\
\hline $\mathrm{Si} 3^{\mathrm{v}}-\mathrm{Cs} 3-\mathrm{O}^{\mathrm{iv}}$ & $116.40(6)$ \\
\hline $\mathrm{Lu} 1-\mathrm{Cs} 3-\mathrm{O} 5^{\mathrm{iv}}$ & $173.83(5)$ \\
\hline $\mathrm{Si} 1^{\mathrm{iv}}-\mathrm{Cs} 3-\mathrm{O}^{\mathrm{iv}}$ & $21.35(6)$ \\
\hline $\mathrm{Cs} 2^{\mathrm{xi}}-\mathrm{Cs} 3-\mathrm{O} 5^{\mathrm{iv}}$ & $44.99(6)$ \\
\hline $\mathrm{Cs} 2-\mathrm{Cs} 3-\mathrm{O}^{\mathrm{iv}}$ & $86.95(6)$ \\
\hline $\mathrm{O} 6^{\mathrm{x}}-\mathrm{Cs} 3-\mathrm{O} 5^{\mathrm{iv}}$ & $110.86(8)$ \\
\hline $\mathrm{O} 7^{\mathrm{xi}}-\mathrm{Cs} 3-\mathrm{O}^{\mathrm{iv}}$ & $72.09(8)$ \\
\hline $\mathrm{Cs} 1^{\mathrm{ix}}-\mathrm{Cs} 3-\mathrm{O} 5^{\mathrm{iv}}$ & $105.54(6)$ \\
\hline $\mathrm{O} 7-\mathrm{Cs} 3-\mathrm{O}^{\mathrm{iv}}$ & $108.90(8)$ \\
\hline $\mathrm{Si} 2^{\mathrm{x}}-\mathrm{Cs} 3-\mathrm{O}^{\mathrm{iv}}$ & $128.38(6)$ \\
\hline $\mathrm{Si} 2-\mathrm{Cs} 3-\mathrm{O} 5^{\mathrm{iv}}$ & $47.77(6)$ \\
\hline $\mathrm{Si} 1-\mathrm{Cs} 3-\mathrm{O} 5^{\mathrm{iv}}$ & $129.50(6)$ \\
\hline $\mathrm{Lu} 11^{\mathrm{i}}-\mathrm{Cs} 3-\mathrm{O} 5^{\mathrm{iv}}$ & $65.06(5)$ \\
\hline $\mathrm{O} 1-\mathrm{Cs} 3-\mathrm{O} 3^{\mathrm{x}}$ & $177.42(10)$ \\
\hline $\mathrm{O} 9^{\mathrm{iv}}-\mathrm{Cs} 3-\mathrm{O} 3^{\mathrm{x}}$ & $81.75(10)$ \\
\hline $\mathrm{O} 5-\mathrm{Cs} 3-\mathrm{O}^{\mathrm{x}}$ & $114.58(10)$ \\
\hline $\mathrm{O} 4-\mathrm{Cs} 3-\mathrm{O} 3^{\mathrm{x}}$ & $114.36(10)$ \\
\hline $\mathrm{O} 3^{\mathrm{ix}}-\mathrm{Cs} 3-\mathrm{O} 3^{\mathrm{x}}$ & $131.40(8)$ \\
\hline $\mathrm{O} 8-\mathrm{Cs} 3-\mathrm{O}^{\mathrm{x}}$ & $68.61(9)$ \\
\hline $\mathrm{O} 6-\mathrm{Cs} 3-\mathrm{O} 3^{\mathrm{x}}$ & $70.28(8)$ \\
\hline $\mathrm{Cs} 1^{\mathrm{x}}-\mathrm{Cs} 3-\mathrm{O} 3^{\mathrm{x}}$ & $40.39(6)$ \\
\hline $\mathrm{Si} 3^{\mathrm{y}}-\mathrm{Cs} 3-\mathrm{O} 3^{\mathrm{x}}$ & $156.99(6)$ \\
\hline $\mathrm{Lu} 1-\mathrm{Cs} 3-\mathrm{O} 3^{\mathrm{x}}$ & $140.28(6)$ \\
\hline $\mathrm{Si} 1^{\mathrm{iv}}-\mathrm{Cs} 3-\mathrm{O}^{\mathrm{x}}$ & $66.05(6)$ \\
\hline $\mathrm{Cs} 2^{\mathrm{xi}}-\mathrm{Cs} 3-\mathrm{O} 3^{\mathrm{x}}$ & $53.56(6)$ \\
\hline $\mathrm{Cs} 2-\mathrm{Cs} 3-\mathrm{O} 3^{\mathrm{x}}$ & $114.39(5)$ \\
\hline $\mathrm{O} 6^{\mathrm{x}}-\mathrm{Cs} 3-\mathrm{O} 3^{\mathrm{x}}$ & $71.59(8)$ \\
\hline $\mathrm{O} 7^{\mathrm{xi}}-\mathrm{Cs} 3-\mathrm{O} 3^{\mathrm{x}}$ & $36.25(8)$ \\
\hline $\mathrm{Cs} 1^{\mathrm{ix}}-\mathrm{Cs} 3-\mathrm{O} 3^{\mathrm{x}}$ & $110.35(5)$ \\
\hline $\mathrm{O} 7-\mathrm{Cs} 3-3^{x}$ & $99.98(8)$ \\
\hline $\mathrm{Si} 2^{x}-\mathrm{Cs} 3-O 3^{x}$ & $94.57(6)$ \\
\hline $\mathrm{Si} 2-\mathrm{Cs} 3-\mathrm{O} 3^{\mathrm{x}}$ & $60.24(6)$ \\
\hline $\mathrm{Si} 1-\mathrm{Cs} 3-\mathrm{O} 3^{\mathrm{x}}$ & $106.31(6)$ \\
\hline
\end{tabular}

$66.14(11)$

$116.10(11)$

$47.76(10)$

$80.27(11)$

$167.63(10)$

$118.38(10)$

$119.34(11)$

$115.30(10)$

$26.94(8)$

$85.11(8)$

$137.25(8)$

$27.18(8)$

$104.17(7)$

$57.13(11)$

$127.58(10)$

$79.78(10)$

$46.44(10)$

$138.69(4)$

$98.89(10)$

$133.55(7)$

$123.02(9)$

$136.76(8)$

$26.49(8)$

$27.61(8)$

$88.50(8)$

$142.07(7)$

$110.74(3)$

67.14 (7)

$25.66(9)$

73.67 (8)

$160.19(8)$

$118.94(8)$

124.88 (8)

$27.02(7)$

100.61 (3)

82.67 (7)

$146.38(5)$

84.33 (9)

140.57 (9)

$100.56(8)$

59.28 (7) 


$$
\begin{aligned}
& \mathrm{O} 2^{\mathrm{ii}}-\mathrm{Cs} 1-\mathrm{Cs} 3^{\mathrm{iii}} \\
& \mathrm{O}^{\mathrm{iv}}-\mathrm{Cs} 1-\mathrm{Cs} 3^{\mathrm{iii}} \\
& \mathrm{Si} 2^{\mathrm{v}}-\mathrm{Cs} 1-\mathrm{Cs} 3^{\mathrm{iii}} \\
& \mathrm{O} 2^{\mathrm{vi}}-\mathrm{Cs} 1-\mathrm{Cs} 3^{\mathrm{iii}} \\
& \mathrm{Si}^{\mathrm{iii}}-\mathrm{Cs} 1-\mathrm{Cs} 3^{\mathrm{iii}} \\
& \mathrm{Si} 3^{\mathrm{iv}}-\mathrm{Cs} 1-\mathrm{Cs} 3^{3 \mathrm{ii}} \\
& \mathrm{O} 3-\mathrm{Cs} 1-\mathrm{Cs} 2 \\
& \mathrm{O} 4-\mathrm{Cs} 1-\mathrm{Cs} 2 \\
& \mathrm{O} 1{ }^{\mathrm{ii}}-\mathrm{Cs} 1-\mathrm{Cs} 2 \\
& \text { O8 }{ }^{i i i}-\mathrm{Cs} 1-\mathrm{Cs} 2 \\
& \mathrm{O} 2^{\mathrm{ii}}-\mathrm{Cs} 1-\mathrm{Cs} 2 \\
& \mathrm{O}^{7 \mathrm{iv}}-\mathrm{Cs} 1-\mathrm{Cs} 2 \\
& \mathrm{Si} 2 \text { - Cs } 1-\mathrm{Cs} 2 \\
& \mathrm{O} 2^{\mathrm{vi}}-\mathrm{Cs} 1-\mathrm{Cs} 2 \\
& \mathrm{Si} 3^{\mathrm{iii}}-\mathrm{Cs} 1-\mathrm{Cs} 2 \\
& \mathrm{Si} 3^{\mathrm{iv}} \text { - Cs } 1 \text { - Cs2 } \\
& \text { Cs }{ }^{3 i i} \text { - Cs } 1-\mathrm{Cs} 2 \\
& \mathrm{O} 3-\mathrm{Cs} 1-\mathrm{Si}^{2 \mathrm{ii}} \\
& \mathrm{O} 4 \text { - } \mathrm{Cs} 1-\mathrm{Si}^{\mathrm{iii}} \\
& \mathrm{O} 1^{\mathrm{ii}}-\mathrm{Cs} 1-\mathrm{Si}_{2}{ }^{\mathrm{iii}} \\
& \mathrm{O} 8^{\mathrm{iii}}-\mathrm{Cs} 1-\mathrm{Si}_{2}{ }^{\mathrm{iii}} \\
& \mathrm{O} 2^{\mathrm{ii}}-\mathrm{Cs} 1-\mathrm{Si}_{2}{ }^{\mathrm{iii}} \\
& \mathrm{O} 7^{\mathrm{iv}}-\mathrm{Cs} 1-\mathrm{Si} 2^{\mathrm{iii}} \\
& \mathrm{Si} 2^{\mathrm{v}}-\mathrm{Cs} 1-\mathrm{Si}_{2}{ }^{\mathrm{iii}} \\
& \mathrm{O} 2^{\mathrm{vi}}-\mathrm{Cs} 1-\mathrm{Si}_{2}{ }^{\mathrm{iii}} \\
& \mathrm{Si}^{\mathrm{iii}}-\mathrm{Cs} 1-\mathrm{Si}_{2}{ }^{\mathrm{iii}} \\
& \mathrm{Si} 3^{\text {iv }}-\mathrm{Cs} 1-\mathrm{Si}_{2}{ }^{\mathrm{iii}} \\
& \mathrm{Cs} 3^{3 i i}-\mathrm{Cs} 1-\mathrm{Si}^{\mathrm{iii}} \\
& \text { Cs2-Cs1- } \mathrm{Si}_{2}{ }^{\mathrm{iii}} \\
& \mathrm{O} 3-\mathrm{Cs} 1-\mathrm{Cs} 3^{\mathrm{i}} \\
& \mathrm{O} 4^{\mathrm{i}}-\mathrm{Cs} 1-\mathrm{Cs} 3^{\mathrm{i}} \\
& \mathrm{O} 1^{\mathrm{ii}}-\mathrm{Cs} 1-\mathrm{Cs} 3^{\mathrm{i}} \\
& \mathrm{O} 8^{\mathrm{iii}}-\mathrm{Cs} 1-\mathrm{Cs} 3^{\mathrm{i}} \\
& \mathrm{O} 2^{\mathrm{ii}}-\mathrm{Cs} 1-\mathrm{Cs} 3^{\mathrm{i}} \\
& \mathrm{O} 7^{\mathrm{iv}}-\mathrm{Cs} 1-\mathrm{Cs} 3^{\mathrm{i}} \\
& \mathrm{Si} 2^{\mathrm{v}}-\mathrm{Cs} 1-\mathrm{Cs} 3^{\mathrm{i}} \\
& \mathrm{O} 2^{\mathrm{vi}}-\mathrm{Cs} 1-\mathrm{Cs} 3^{\mathrm{i}} \\
& \mathrm{Si} 3^{3 i i}-\mathrm{Cs} 1-\mathrm{Cs}^{\mathrm{i}}{ }^{\mathrm{i}} \\
& \mathrm{Si}^{\mathrm{iv}}-\mathrm{Cs} 1-\mathrm{Cs}^{\mathrm{i}} \\
& \mathrm{Cs} 3^{3 i i}-\mathrm{Cs} 1-\mathrm{Cs}^{\mathrm{i}}{ }^{\mathrm{i}} \\
& \mathrm{Cs} 2-\mathrm{Cs} 1-\mathrm{Cs} 3^{\mathrm{i}} \\
& \mathrm{Si} 2^{\mathrm{iii}}-\mathrm{Cs} 1-\mathrm{Cs} 3^{\mathrm{i}} \\
& \mathrm{O} 3-\mathrm{Cs} 1-\mathrm{Lu} 1^{\mathrm{ii}}
\end{aligned}
$$

\begin{tabular}{|c|c|c|}
\hline $126.61(7)$ & $\mathrm{Lu} 1^{\mathrm{i}}-\mathrm{Cs} 3-\mathrm{O} 3^{\mathrm{x}}$ & $106.38(6)$ \\
\hline $67.16(7)$ & $\mathrm{O} 5^{\text {iv }}-\mathrm{Cs} 3-\mathrm{O} 3^{\mathrm{x}}$ & $45.64(8)$ \\
\hline $145.59(3)$ & $\mathrm{O} 1-\mathrm{Cs} 3-\mathrm{Si} 3$ & $99.62(8)$ \\
\hline $80.65(6)$ & $\mathrm{O} 9^{\text {iv }}-\mathrm{Cs} 3-\mathrm{Si} 3$ & $106.83(8)$ \\
\hline $75.63(3)$ & $\mathrm{O} 5-\mathrm{Cs} 3-\mathrm{Si} 3$ & $60.20(8)$ \\
\hline $85.49(3)$ & $\mathrm{O} 4-\mathrm{Cs} 3-\mathrm{Si} 3$ & $120.26(8)$ \\
\hline $131.73(8)$ & $\mathrm{O} 3^{\mathrm{ix}}-\mathrm{Cs} 3-\mathrm{Si} 3$ & $134.44(8)$ \\
\hline $73.65(8)$ & $\mathrm{O} 8-\mathrm{Cs} 3-\mathrm{Si} 3$ & $18.99(7)$ \\
\hline $92.56(8)$ & $\mathrm{O} 6-\mathrm{Cs} 3-\mathrm{Si} 3$ & $60.75(6)$ \\
\hline $113.70(7)$ & $\mathrm{Cs} 1^{\mathrm{x}}-\mathrm{Cs} 3-\mathrm{Si} 3$ & $51.62(2)$ \\
\hline $50.14(8)$ & $\mathrm{Si} 3{ }^{v}-\mathrm{Cs} 3-\mathrm{Si} 3$ & $117.585(15)$ \\
\hline $84.35(7)$ & $\mathrm{Lu} 1-\mathrm{Cs} 3-\mathrm{Si} 3$ & $94.51(2)$ \\
\hline $64.42(3)$ & $\mathrm{Si} 1^{\mathrm{iv}}-\mathrm{Cs} 3-\mathrm{Si} 3$ & $85.87(3)$ \\
\hline $158.76(6)$ & $\mathrm{Cs} 2^{\mathrm{xi}}-\mathrm{Cs} 3-\mathrm{Si} 3$ & $128.83(2)$ \\
\hline $97.44(3)$ & $\mathrm{Cs} 2-\mathrm{Cs} 3-\mathrm{Si} 3$ & $51.43(2)$ \\
\hline $107.05(3)$ & $\mathrm{O} 6^{x}-\mathrm{Cs} 3-\mathrm{Si} 3$ & $109.18(6)$ \\
\hline $81.354(14)$ & $\mathrm{O} 7^{\mathrm{xi}}-\mathrm{Cs} 3-\mathrm{Si} 3$ & $105.86(6)$ \\
\hline $75.86(9)$ & $\mathrm{Cs} 1^{\mathrm{ix}}-\mathrm{Cs} 3-\mathrm{Si} 3$ & $166.04(2)$ \\
\hline $149.52(9)$ & $\mathrm{O} 7-\mathrm{Cs} 3-\mathrm{Si} 3$ & $21.92(6)$ \\
\hline $66.62(8)$ & $\mathrm{Si} 2^{x}-\mathrm{Cs} 3-\mathrm{Si} 3$ & $120.30(3)$ \\
\hline $22.99(8)$ & $\mathrm{Si} 2-\mathrm{Cs} 3-\mathrm{Si} 3$ & $41.49(3)$ \\
\hline $132.67(8)$ & $\mathrm{Si} 1-\mathrm{Cs} 3-\mathrm{Si} 3$ & $41.37(3)$ \\
\hline $107.56(7)$ & $\mathrm{Lu} 11^{\mathrm{i}}-\mathrm{Cs} 3-\mathrm{Si} 3$ & $124.39(2)$ \\
\hline $143.711(19)$ & $\mathrm{O} 5^{\text {iv }}-\mathrm{Cs} 3-\mathrm{Si} 3$ & $88.38(6)$ \\
\hline $23.80(7)$ & $\mathrm{O}^{\mathrm{x}}-\mathrm{Cs} 3-\mathrm{Si} 3$ & $80.05(6)$ \\
\hline $47.22(3)$ & $\mathrm{O} 1-\mathrm{Cs} 3-\mathrm{O} 2^{\mathrm{iv}}$ & $140.96(10)$ \\
\hline $99.69(3)$ & $\mathrm{O} 9^{\mathrm{iv}}-\mathrm{Cs} 3-\mathrm{O} 2^{\mathrm{iv}}$ & $70.26(10)$ \\
\hline $65.73(2)$ & $\mathrm{O} 5-\mathrm{Cs} 3-\mathrm{O} 2^{\mathrm{iv}}$ & $108.64(9)$ \\
\hline $135.44(3)$ & $\mathrm{O} 4-\mathrm{Cs} 3-\mathrm{O} 2^{\mathrm{iv}}$ & $149.71(10)$ \\
\hline $55.21(9)$ & $\mathrm{O} 3^{\mathrm{ix}}-\mathrm{Cs} 3-\mathrm{O} 2^{\mathrm{iv}}$ & $127.68(9)$ \\
\hline $49.57(8)$ & $\mathrm{O} 8-\mathrm{Cs} 3-\mathrm{O} 2^{\mathrm{iv}}$ & $33.98(8)$ \\
\hline $109.01(8)$ & $\mathrm{O} 6-\mathrm{Cs} 3-\mathrm{O}^{\mathrm{iv}}$ & $34.93(8)$ \\
\hline $121.27(7)$ & $\mathrm{Cs} 1^{\mathrm{x}}-\mathrm{Cs} 3-\mathrm{O} 2^{\mathrm{iv}}$ & $49.04(6)$ \\
\hline $91.10(7)$ & $\mathrm{Si} 3^{\mathrm{v}}-\mathrm{Cs} 3-\mathrm{O} 2^{\mathrm{iv}}$ & $139.09(6)$ \\
\hline $82.54(7)$ & $\mathrm{Lu} 1-\mathrm{Cs} 3-\mathrm{O} 2^{\mathrm{iv}}$ & $145.27(5)$ \\
\hline $65.80(3)$ & $\mathrm{Si} 1^{\mathrm{iv}}-\mathrm{Cs} 3-\mathrm{O} 2^{\mathrm{iv}}$ & $46.50(6)$ \\
\hline $78.16(6)$ & $\mathrm{Cs} 2^{\mathrm{xi}}-\mathrm{Cs} 3-\mathrm{O} 2^{\mathrm{iv}}$ & $77.70(5)$ \\
\hline $125.02(3)$ & $\mathrm{Cs} 2-\mathrm{Cs} 3-\mathrm{O} 2^{\mathrm{iv}}$ & $74.01(5)$ \\
\hline $57.95(2)$ & $\mathrm{O} 6^{\mathrm{x}}-\mathrm{Cs} 3-\mathrm{O} 2^{\mathrm{iv}}$ & $108.15(8)$ \\
\hline $139.522(15)$ & $\mathrm{O} 7^{\mathrm{xi}}-\mathrm{Cs} 3-\mathrm{O} 2^{\mathrm{iv}}$ & $76.54(8)$ \\
\hline $123.056(15)$ & $\mathrm{Cs} 1^{\mathrm{ix}}-\mathrm{Cs} 3-\mathrm{O} 2^{\mathrm{iv}}$ & $142.26(5)$ \\
\hline $101.27(2)$ & $\mathrm{O} 7-\mathrm{Cs} 3-\mathrm{O} 2^{\mathrm{iv}}$ & $73.28(8)$ \\
\hline $158.89(9)$ & $\mathrm{Si} 2^{\mathrm{x}}-\mathrm{Cs} 3-\mathrm{O} 2^{\mathrm{iv}}$ & $131.79(6)$ \\
\hline $83.27(8)$ & $\mathrm{Si} 2-\mathrm{Cs} 3-\mathrm{O} 2^{\mathrm{iv}}$ & $19.87(6)$ \\
\hline $33.77(8)$ & $\mathrm{Si} 1-\mathrm{Cs} 3-\mathrm{O} 2^{\mathrm{iv}}$ & $91.25(6)$ \\
\hline $83.25(7)$ & $\mathrm{Lu} 11^{\mathrm{i}}-\mathrm{Cs} 3-\mathrm{O} 2^{\mathrm{iv}}$ & $98.25(5)$ \\
\hline $33.03(8)$ & $\mathrm{O} 5^{\mathrm{iv}}-\mathrm{Cs} 3-\mathrm{O} 2^{\mathrm{iv}}$ & $38.83(8)$ \\
\hline $148.51(7)$ & $\mathrm{O} 3^{\mathrm{x}}-\mathrm{Cs} 3-\mathrm{O} 2^{\mathrm{iv}}$ & $40.38(7)$ \\
\hline
\end{tabular}

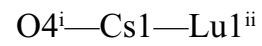

$$
\begin{aligned}
& \mathrm{O} 1^{\mathrm{ii}}-\mathrm{Cs} 1-\mathrm{Lu} 1^{\mathrm{ii}} \\
& \text { O8 }{ }^{\mathrm{iii}}-\mathrm{Cs} 1-\mathrm{Lu} 1^{\mathrm{ii}} \\
& \mathrm{O} 2^{\mathrm{ii}}-\mathrm{Cs} 1-\mathrm{Lu} 1^{\mathrm{ii}} \\
& \mathrm{O}^{\mathrm{iv}}-\mathrm{Cs} 1-\mathrm{Lu} 1^{\mathrm{ii}}
\end{aligned}
$$




\begin{tabular}{|c|c|c|c|}
\hline $\mathrm{Si} 2^{\mathrm{v}}-\mathrm{Cs} 1-\mathrm{Lu} 1^{\mathrm{ii}}$ & $56.33(2)$ & $\mathrm{Si} 3-\mathrm{Cs} 3-\mathrm{O} 2^{\mathrm{iv}}$ & $51.49(6)$ \\
\hline $\mathrm{O} 2^{\mathrm{vi}}-\mathrm{Cs} 1-\mathrm{Lu} 1^{\mathrm{ii}}$ & $112.41(7)$ & $\mathrm{O} 3^{\mathrm{xii}-\mathrm{Lu} 1-\mathrm{O} 2}$ & $90.72(17)$ \\
\hline $\mathrm{Si} 3^{\mathrm{iii}}-\mathrm{Cs} 1-\mathrm{Lu} 1^{\mathrm{ii}}$ & $55.64(2)$ & 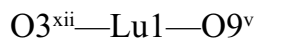 & $89.23(16)$ \\
\hline $\mathrm{Si}^{\mathrm{iv}}-\mathrm{Cs} 1-\mathrm{Lu} 1^{\mathrm{ii}}$ & $156.94(3)$ & $\mathrm{O} 2-\mathrm{Lu} 1-\mathrm{O} 9^{\mathrm{v}}$ & $87.41(15)$ \\
\hline $\mathrm{Cs} 3^{\mathrm{iii}}-\mathrm{Cs} 1-\mathrm{Lu} 1^{\mathrm{ii}}$ & $113.351(13)$ & $\mathrm{O} 3^{\mathrm{xii}-\mathrm{Lu} 1-\mathrm{O} 4}$ & $84.26(15)$ \\
\hline $\mathrm{Cs} 2-\mathrm{Cs} 1-\mathrm{Lu} 1^{\mathrm{ii}}$ & $65.251(10)$ & $\mathrm{O} 2-\mathrm{Lu} 1-\mathrm{O} 4$ & $171.01(16)$ \\
\hline $\mathrm{Si} 2^{\mathrm{iii}}-\mathrm{Cs} 1-\mathrm{Lu} 1^{\mathrm{ii}}$ & $100.24(2)$ & $\mathrm{O} 9^{\mathrm{v}}-\mathrm{Lu} 1-\mathrm{O} 4$ & $99.95(16)$ \\
\hline $\mathrm{Cs} 3{ }^{\mathrm{i}}-\mathrm{Cs} 1-\mathrm{Lu} 1^{1 \mathrm{ii}}$ & $106.540(13)$ & $\mathrm{O} 3^{\mathrm{xii}-\mathrm{Lu} 1-\mathrm{O} 5}$ & $99.47(17)$ \\
\hline $\mathrm{O} 3-\mathrm{Cs} 1-\mathrm{O} 9^{i \mathrm{iii}}$ & $167.84(11)$ & $\mathrm{O} 2-\mathrm{Lu} 1-\mathrm{O} 5$ & $85.00(15)$ \\
\hline $\mathrm{O} 4^{\mathrm{i}}-\mathrm{Cs} 1-\mathrm{O} 9^{\mathrm{iii}}$ & $99.76(10)$ & $\mathrm{O} 9^{\mathrm{v}}-\mathrm{Lu} 1-\mathrm{O} 5$ & $168.50(16)$ \\
\hline $\mathrm{O} 1^{\mathrm{ii}}-\mathrm{Cs} 1-\mathrm{O} 9^{\mathrm{iii}}$ & $47.21(10)$ & $\mathrm{O} 4-\mathrm{Lu} 1-\mathrm{O} 5$ & $88.46(16)$ \\
\hline $\mathrm{O} 8^{\mathrm{iii}}-\mathrm{Cs} 1-\mathrm{O} 9^{\mathrm{iii}}$ & $75.96(9)$ & 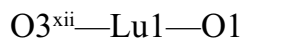 & $169.68(16)$ \\
\hline $\mathrm{O} 2^{\mathrm{ii}}-\mathrm{Cs} 1-\mathrm{O} 9^{\mathrm{iii}}$ & $48.52(9)$ & $\mathrm{O} 2-\mathrm{Lu} 1-\mathrm{O} 1$ & $96.45(16)$ \\
\hline $\mathrm{O} 7^{\mathrm{iv}}-\mathrm{Cs} 1-\mathrm{O} 9^{\mathrm{iii}}$ & $126.54(9)$ & $\mathrm{O} 9^{v}-\mathrm{Lu} 1-\mathrm{O} 1$ & $83.71(14)$ \\
\hline $\mathrm{Si} 2^{\mathrm{v}}-\mathrm{Cs} 1-\mathrm{O} 9^{\mathrm{iii}}$ & $75.64(6)$ & $\mathrm{O} 4-\mathrm{Lu} 1-\mathrm{O} 1$ & $89.54(15)$ \\
\hline $\mathrm{O} 2^{\mathrm{vi}}-\mathrm{Cs} 1-\mathrm{O}^{9 \mathrm{iii}}$ & $119.97(9)$ & $\mathrm{O} 5-\mathrm{Lu} 1-\mathrm{O} 1$ & $88.57(16)$ \\
\hline $\mathrm{Si} 3^{\mathrm{iii}}-\mathrm{Cs} 1-\mathrm{O} 9^{\mathrm{iii}}$ & $52.85(6)$ & $\mathrm{O}^{\mathrm{xii}}-\mathrm{Lu} 1-\mathrm{Cs} 3$ & $128.68(11)$ \\
\hline $\mathrm{Si} 3^{\mathrm{iv}}-\mathrm{Cs} 1-\mathrm{O} 9^{\mathrm{iii}}$ & $152.56(7)$ & $\mathrm{O} 2-\mathrm{Lu} 1-\mathrm{Cs} 3$ & $124.41(12)$ \\
\hline $\mathrm{Cs} 3^{\mathrm{iii}}-\mathrm{Cs} 1-\mathrm{O} 9^{\mathrm{iii}}$ & $83.52(6)$ & $\mathrm{O} 9^{v}-\mathrm{Lu} 1-\mathrm{Cs} 3$ & $124.34(10)$ \\
\hline $\mathrm{Cs} 2-\mathrm{Cs} 1-\mathrm{O}^{\mathrm{iii}}$ & $46.40(6)$ & $\mathrm{O} 4-\mathrm{Lu} 1-\mathrm{Cs} 3$ & $55.15(11)$ \\
\hline $\mathrm{Si} 2^{\mathrm{iii}}-\mathrm{Cs} 1-\mathrm{O} 9^{\mathrm{iii}}$ & $98.55(6)$ & $\mathrm{O} 5-\mathrm{Lu} 1-\mathrm{Cs} 3$ & $54.89(11)$ \\
\hline $\mathrm{Cs} 3^{\mathrm{i}}-\mathrm{Cs} 1-\mathrm{O} 9^{\mathrm{iii}}$ & $136.95(6)$ & $\mathrm{O} 1-\mathrm{Lu} 1-\mathrm{Cs} 3$ & $51.82(10)$ \\
\hline $\mathrm{Lu} 1^{\mathrm{ii}}-\mathrm{Cs} 1-\mathrm{O} 9^{\mathrm{iii}}$ & $31.85(6)$ & $\mathrm{O} 3^{\mathrm{xii}}-\mathrm{Lu} 1-\mathrm{Cs} 1^{\mathrm{xiv}}$ & $136.55(11)$ \\
\hline $\mathrm{O} 3-\mathrm{Cs} 1-\mathrm{O}^{\mathrm{i}}$ & $41.66(10)$ & $\mathrm{O} 2-\mathrm{Lu} 1-\mathrm{Cs} 1^{\mathrm{xiv}}$ & $49.32(12)$ \\
\hline $\mathrm{O} 4-\mathrm{Cs} 1-\mathrm{O} 1^{\mathrm{i}}$ & $50.75(9)$ & O9v- $9^{v} 1-\mathrm{Cs}^{\mathrm{xiv}}$ & $74.72(11)$ \\
\hline $\mathrm{O} 1^{\mathrm{ii}}-\mathrm{Cs} 1-\mathrm{O} 1^{\mathrm{i}}$ & $151.28(13)$ & $\mathrm{O} 4-\mathrm{Lu} 1-\mathrm{Cs} 1^{\mathrm{xiv}}$ & $137.59(10)$ \\
\hline 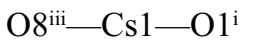 & $138.93(10)$ & O5-Lu1-Cs $1^{\text {xiv }}$ & $93.78(12)$ \\
\hline $\mathrm{O} 2^{\mathrm{ii}}-\mathrm{Cs} 1-\mathrm{O} 1^{\mathrm{i}}$ & $103.12(9)$ & $\mathrm{O} 1-\mathrm{Lu} 1-\mathrm{Cs} 1^{\mathrm{xiv}}$ & $48.28(11)$ \\
\hline $\mathrm{O} 7^{\mathrm{iv}}-\mathrm{Cs} 1-\mathrm{O}^{\mathrm{i}}$ & $40.89(8)$ & $\mathrm{Cs} 3-\mathrm{Lu} 1-\mathrm{Cs} 1^{\mathrm{xiv}}$ & $92.527(12)$ \\
\hline $\mathrm{Si} 2^{\mathrm{v}}-\mathrm{Cs} 1-\mathrm{O} 1^{\mathrm{i}}$ & $77.64(6)$ & $\mathrm{O} 3^{\mathrm{xii}}-\mathrm{Lu} 1-\mathrm{Cs} 2^{\mathrm{xiv}}$ & $85.11(11)$ \\
\hline $\mathrm{O} 2^{\mathrm{vi}}-\mathrm{Cs} 1-\mathrm{O} 1^{\mathrm{i}}$ & $95.63(9)$ & $\mathrm{O} 2-\mathrm{Lu} 1-\mathrm{Cs} 2^{\mathrm{xiv}}$ & $42.36(10)$ \\
\hline $\mathrm{Si} 3^{\mathrm{iii}}-\mathrm{Cs} 1-\mathrm{O} 1^{\mathrm{i}}$ & $162.28(6)$ & O9v-Lu1-Cs $2^{\text {xiv }}$ & $45.48(11)$ \\
\hline $\mathrm{Si}^{\mathrm{iv}}-\mathrm{Cs} 1-\mathrm{O} 1^{\mathrm{i}}$ & $23.30(6)$ & $\mathrm{O} 4-\mathrm{Lu} 1-\mathrm{Cs} 2^{\mathrm{xiv}}$ & $143.89(12)$ \\
\hline $\mathrm{Cs} 3^{3 \mathrm{ii}}-\mathrm{Cs} 1-\mathrm{O} 1^{\mathrm{i}}$ & $106.68(6)$ & $\mathrm{O} 5-\mathrm{Lu} 1-\mathrm{Cs} 2^{\mathrm{xiv}}$ & $127.35(11)$ \\
\hline $\mathrm{Cs} 2-\mathrm{Cs} 1-\mathrm{O} 1^{\mathrm{i}}$ & $100.27(6)$ & $\mathrm{O} 1-\mathrm{Lu} 1-\mathrm{Cs} 2^{\mathrm{xiv}}$ & $95.18(11)$ \\
\hline $\mathrm{Si} 2^{\mathrm{iii}}-\mathrm{Cs} 1-\mathrm{O} 1^{\mathrm{i}}$ & $116.95(6)$ & $\mathrm{Cs} 3-\mathrm{Lu} 1-\mathrm{Cs} 2^{\mathrm{xiv}}$ & $146.201(13)$ \\
\hline $\mathrm{Cs} 3^{\mathrm{i}}-\mathrm{Cs} 1-\mathrm{O} 1^{\mathrm{i}}$ & $42.79(6)$ & $\mathrm{Cs} 1^{\mathrm{xiv}}-\mathrm{Lu} 1-\mathrm{Cs} 2^{\mathrm{xiv}}$ & $54.773(11)$ \\
\hline $\mathrm{Lu} 1^{\mathrm{ii}}-\mathrm{Cs} 1-\mathrm{O} 1^{\mathrm{i}}$ & $133.81(6)$ & 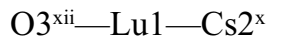 & $60.24(12)$ \\
\hline $\mathrm{O} 9^{\mathrm{iii}}-\mathrm{Cs} 1-\mathrm{O} 1^{\mathrm{i}}$ & $144.29(8)$ & $\mathrm{O} 2-\mathrm{Lu} 1-\mathrm{Cs} 2^{\mathrm{x}}$ & $106.33(10)$ \\
\hline $\mathrm{O} 3-\mathrm{Cs} 1-\mathrm{O} 6$ & $86.68(10)$ & $\mathrm{O} 9^{\mathrm{v}}-\mathrm{Lu} 1-\mathrm{Cs} 2^{\mathrm{x}}$ & $145.93(11)$ \\
\hline $\mathrm{O} 4-\mathrm{Cs} 1-\mathrm{O} 6$ & $81.18(11)$ & $\mathrm{O} 4-\mathrm{Lu} 1-\mathrm{Cs} 2^{\mathrm{x}}$ & $64.69(11)$ \\
\hline $\mathrm{O} 1 \mathrm{ii}-\mathrm{Cs} 1-\mathrm{O} 6$ & $134.06(9)$ & $\mathrm{O} 5-\mathrm{Lu} 1-\mathrm{Cs} 2^{\mathrm{x}}$ & $45.24(12)$ \\
\hline $\mathrm{O} 88^{\mathrm{iii}}-\mathrm{Cs} 1-\mathrm{O} 6$ & $117.91(9)$ & $\mathrm{O} 1-\mathrm{Lu} 1-\mathrm{Cs} 2^{\mathrm{x}}$ & $124.05(10)$ \\
\hline $\mathrm{O} 22^{\mathrm{ii}}-\mathrm{Cs} 1-\mathrm{O} 6$ & $91.97(10)$ & $\mathrm{Cs} 3-\mathrm{Lu} 1-\mathrm{Cs} 2^{\mathrm{x}}$ & $73.508(9)$ \\
\hline $\mathrm{O}^{\mathrm{iv}}-\mathrm{Cs} 1-\mathrm{O} 6$ & $39.11(9)$ & $\mathrm{Cs} 1^{\mathrm{xiv}}-\mathrm{Lu} 1-\mathrm{Cs} 2^{\mathrm{x}}$ & $137.582(10)$ \\
\hline $\mathrm{Si} 2^{\mathrm{v}}-\mathrm{Cs} 1-\mathrm{O} 6$ & $92.07(7)$ & $\mathrm{Cs} 2^{\mathrm{xiv}}-\mathrm{Lu} 1-\mathrm{Cs} 2^{\mathrm{x}}$ & $135.276(14)$ \\
\hline $\mathrm{O} 2^{\mathrm{vi}}-\mathrm{Cs} 1-\mathrm{O} 6$ & $129.18(9)$ & $\mathrm{O} 3^{\mathrm{xii}}-\mathrm{Lu} 1-\mathrm{Cs} 3^{\mathrm{ix}}$ & $49.34(12)$ \\
\hline $\mathrm{Si} 3^{\mathrm{iii}}-\mathrm{Cs} 1-\mathrm{O} 6$ & $123.58(6)$ & $\mathrm{O} 2-\mathrm{Lu} 1-\mathrm{Cs} 3^{\mathrm{ix}}$ & $87.93(11)$ \\
\hline
\end{tabular}




$$
\begin{aligned}
& \mathrm{Si} 3^{\mathrm{iv}}-\mathrm{Cs} 1-\mathrm{O} 6 \\
& \text { Cs } 3 \text { iii-Cs } 1-\mathrm{O} 6 \\
& \text { Cs2-Cs1-O6 }
\end{aligned}
$$

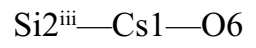

$$
\begin{aligned}
& \text { Cs } 3^{\mathrm{i}}-\mathrm{Cs} 1-\mathrm{O} 6 \\
& \mathrm{Lu} 1^{\mathrm{ii}}-\mathrm{Cs} 1-\mathrm{O} 6 \\
& \text { O9iii-Cs1-O6 } \\
& \mathrm{O} 1 \text { - } \mathrm{Cs} 1-\mathrm{O} 6 \\
& \mathrm{O} 3-\mathrm{Cs} 1-\mathrm{O}^{\text {iii }} \\
& \mathrm{O} 4-\mathrm{Cs} 1-\mathrm{O} 5^{\mathrm{iii}}
\end{aligned}
$$

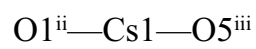

\begin{tabular}{|c|c|}
\hline $65.15(6)$ & $\mathrm{O} 9^{\mathrm{v}}-\mathrm{Lu} 1-\mathrm{Cs} 3^{\mathrm{ix}}$ \\
\hline $59.59(6)$ & $\mathrm{O} 4-\mathrm{Lu} 1-\mathrm{Cs} 3^{\mathrm{ix}}$ \\
\hline $46.65(6)$ & $\mathrm{O} 5-\mathrm{Lu} 1-\mathrm{Cs} 3^{\mathrm{ix}}$ \\
\hline $123.81(7)$ & $\mathrm{O} 1-\mathrm{Lu} 1-\mathrm{Cs} 3^{\mathrm{ix}}$ \\
\hline $111.38(5)$ & Cs3-Lu1-Cs $3^{i x}$ \\
\hline $111.84(6)$ & $\mathrm{Cs} 1^{\mathrm{xiv}}-\mathrm{Lu} 1-\mathrm{Cs} 3^{\mathrm{ix}}$ \\
\hline $87.65(8)$ & $\mathrm{Cs} 2^{\mathrm{xiv}}-\mathrm{Lu} 1-\mathrm{Cs} 3^{\mathrm{ix}}$ \\
\hline $70.04(8)$ & $\mathrm{Cs} 2^{\mathrm{x}}-\mathrm{Lu} 1-\mathrm{Cs} 3^{\mathrm{ix}}$ \\
\hline $129.64(11)$ & $\mathrm{O} 3^{\mathrm{xii}}-\mathrm{Lu} 1-\mathrm{Cs} 1^{\mathrm{xii}}$ \\
\hline $120.73(10)$ & $\mathrm{O} 2-\mathrm{Lu} 1-\mathrm{Cs} 1^{\mathrm{xii}}$ \\
\hline $73.63(10)$ & O9v-Lu1-Cs1 $1^{\mathrm{xii}}$ \\
\hline $67.96(10)$ & $\mathrm{O} 4-\mathrm{Lu} 1-\mathrm{Cs} 1^{\mathrm{xii}}$ \\
\hline $81.39(9)$ & $\mathrm{O} 5-\mathrm{Lu} 1-\mathrm{Cs} 1^{\mathrm{xii}}$ \\
\hline $95.73(9)$ & $\mathrm{O} 1-\mathrm{Lu} 1-\mathrm{Cs} 1^{\mathrm{xii}}$ \\
\hline $105.63(7)$ & $\mathrm{Cs} 3-\mathrm{Lu} 1-\mathrm{Cs} 1^{\mathrm{xii}}$ \\
\hline $111.55(9)$ & $\mathrm{Cs} 1^{\mathrm{xiv}}-\mathrm{Lu} 1-\mathrm{Cs} 1^{\mathrm{xii}}$ \\
\hline $61.28(7)$ & $\mathrm{Cs} 2^{\mathrm{xiv}}-\mathrm{Lu} 1-\mathrm{Cs} 1^{\mathrm{xii}}$ \\
\hline $121.93(6)$ & $\mathrm{Cs} 2^{\mathrm{x}}-\mathrm{Lu} 1-\mathrm{Cs} 1^{\mathrm{xii}}$ \\
\hline $46.12(6)$ & $\mathrm{Cs} 3^{\mathrm{ix}}-\mathrm{Lu} 1-\mathrm{Cs} 1^{\mathrm{xii}}$ \\
\hline $47.24(6)$ & $\mathrm{O} 5-\mathrm{Si} 1-\mathrm{O} 9$ \\
\hline $88.37(7)$ & $\mathrm{O} 5-\mathrm{Si} 1-\mathrm{O} 7$ \\
\hline $170.29(7)$ & $\mathrm{O} 9-\mathrm{Si1}-\mathrm{O} 7$ \\
\hline $70.18(6)$ & $\mathrm{O} 5-\mathrm{Si} 1-\mathrm{O}^{\mathrm{vii}}$ \\
\hline $38.52(8)$ & $\mathrm{O} 9-\mathrm{Si} 1-\mathrm{O}^{\mathrm{vii}}$ \\
\hline $133.04(9)$ & $\mathrm{O} 7-\mathrm{Si} 1-\mathrm{O}^{\mathrm{vii}}$ \\
\hline $63.06(8)$ & $\mathrm{O} 5-\mathrm{Si} 1-\mathrm{Cs} 2^{\mathrm{x}}$ \\
\hline $133.13(11)$ & $\mathrm{O} 9-\mathrm{Si} 1-\mathrm{Cs} 2^{\mathrm{x}}$ \\
\hline $136.79(10)$ & $\mathrm{O} 7-\mathrm{Si} 1-\mathrm{Cs} 2^{\mathrm{x}}$ \\
\hline $40.17(9)$ & $\mathrm{O}^{\mathrm{vii}}-\mathrm{Si} 1-\mathrm{Cs} 2^{\mathrm{x}}$ \\
\hline $38.49(9)$ & $\mathrm{O} 5-\mathrm{Si} 1-\mathrm{Cs} 3^{\mathrm{vii}}$ \\
\hline $83.98(10)$ & $\mathrm{O} 9-\mathrm{Si} 1-\mathrm{Cs} 3^{\mathrm{vii}}$ \\
\hline $127.58(11)$ & $\mathrm{O} 7-\mathrm{Si} 1-\mathrm{Cs} 3^{\mathrm{vii}}$ \\
\hline $110.60(6)$ & $\mathrm{O}^{\mathrm{vii}}-\mathrm{Si} 1-\mathrm{Cs} 3^{\mathrm{vii}}$ \\
\hline $84.53(9)$ & $\mathrm{Cs} 2^{\mathrm{x}}-\mathrm{Si} 1-\mathrm{Cs} 3^{\mathrm{vii}}$ \\
\hline $23.21(6)$ & $\mathrm{O} 5-\mathrm{Si} 1-\mathrm{Cs} 3$ \\
\hline $146.46(6)$ & O9-Si1-Cs3 \\
\hline $61.85(6)$ & $\mathrm{O} 7-\mathrm{Si} 1-\mathrm{Cs} 3$ \\
\hline $77.07(6)$ & $\mathrm{O}^{\mathrm{vii}}-\mathrm{Si} 1-\mathrm{Cs} 3$ \\
\hline $61.46(6)$ & $\mathrm{Cs} 2^{\mathrm{x}}-\mathrm{Si} 1-\mathrm{Cs} 3$ \\
\hline $147.53(6)$ & $\mathrm{Cs} 3^{\mathrm{vii}}-\mathrm{Si} 1-\mathrm{Cs} 3$ \\
\hline $55.59(6)$ & $\mathrm{O} 5-\mathrm{Si} 1-\mathrm{Cs} 1^{\mathrm{x}}$ \\
\hline $38.09(8)$ & O9-Si1-Cs1 $1^{x}$ \\
\hline $168.40(8)$ & $\mathrm{O} 7-\mathrm{Si} 1-\mathrm{Cs}^{\mathrm{x}}$ \\
\hline $100.87(8)$ & $\mathrm{O}^{\mathrm{vii}}-\mathrm{Si} 1-\mathrm{Cs} 1^{\mathrm{x}}$ \\
\hline $38.11(8)$ & $\mathrm{Cs} 2^{\mathrm{x}}-\mathrm{Si} 1-\mathrm{Cs} 1^{\mathrm{x}}$ \\
\hline $78.50(8)$ & $\mathrm{Cs} 3^{\mathrm{vii}}-\mathrm{Si} 1-\mathrm{Cs} 1^{\mathrm{x}}$ \\
\hline $94.13(9)$ & $\mathrm{Cs} 3-\mathrm{Si} 1-\mathrm{Cs} 1^{\mathrm{x}}$ \\
\hline $61.44(8)$ & $\mathrm{O} 5-\mathrm{Si} 1-\mathrm{Cs} 2^{\mathrm{vii}}$ \\
\hline
\end{tabular}

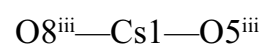



$$
\begin{aligned}
& \mathrm{O} 7^{\mathrm{iv}}-\mathrm{Cs} 1-\mathrm{O} 5^{\mathrm{iii}} \\
& \mathrm{Si} 2^{\mathrm{v}}-\mathrm{Cs} 1-\mathrm{O} 5^{\mathrm{iii}} \\
& \mathrm{O} 2^{\mathrm{vi}}-\mathrm{Cs} 1-\mathrm{O} 5^{\mathrm{iii}} \\
& \mathrm{Si} 3^{\mathrm{iii}}-\mathrm{Cs} 1-\mathrm{O} 5^{\mathrm{iii}} \\
& \mathrm{Si}^{\text {iv }}-\mathrm{Cs} 1-\mathrm{O} 5^{\mathrm{iii}} \\
& \mathrm{Cs} 3^{3 i i}-\mathrm{Cs} 1-\mathrm{O} 5^{\mathrm{iii}} \\
& \mathrm{Cs} 2-\mathrm{Cs} 1-\mathrm{O}^{\mathrm{iii}} \\
& \mathrm{Si} 2^{\mathrm{iii}}-\mathrm{Cs} 1-\mathrm{O} 5^{\mathrm{iii}} \\
& \mathrm{Cs} 3^{\mathrm{i}}-\mathrm{Cs} 1-\mathrm{O}^{\mathrm{iii}} \\
& \mathrm{Lu} 1^{\mathrm{ii}}-\mathrm{Cs} 1-\mathrm{O} 5^{\mathrm{iii}} \\
& \text { O9iii- } \mathrm{Cs} 1-\mathrm{O}^{\mathrm{iii}} \\
& \mathrm{O} 1^{\mathrm{i}}-\mathrm{Cs} 1-\mathrm{O} 5^{\mathrm{iii}} \\
& \text { O6- } \mathrm{Cs} 1-\mathrm{O} 5^{\mathrm{iii}} \\
& \mathrm{O} 3-\mathrm{Cs} 1-\mathrm{O} 7^{\mathrm{iii}} \\
& \mathrm{O} 4-\mathrm{Cs} 1-\mathrm{O} 7^{\mathrm{iii}} \\
& \mathrm{O} 1^{1 i}-\mathrm{Cs} 1-\mathrm{O} 7^{\mathrm{iii}} \\
& \mathrm{O} 8^{\mathrm{iii}}-\mathrm{Cs} 1-\mathrm{O} 7^{\mathrm{iii}} \\
& \mathrm{O} 2^{\mathrm{ii}}-\mathrm{Cs} 1-\mathrm{O} 7^{\mathrm{iii}} \\
& \mathrm{O} 7^{\mathrm{iv}}-\mathrm{Cs} 1-\mathrm{O} 7^{\mathrm{iii}} \\
& \mathrm{Si} 2^{\mathrm{v}}-\mathrm{Cs} 1-\mathrm{O} 7^{\mathrm{iii}} \\
& \mathrm{O}^{\mathrm{vi}}-\mathrm{Cs} 1-\mathrm{O} 7^{\mathrm{iii}} \\
& \mathrm{Si} 3^{i i i}-\mathrm{Cs} 1-\mathrm{O} 7^{\mathrm{iii}} \\
& \mathrm{Si} 3^{\mathrm{iv}}-\mathrm{Cs} 1-\mathrm{O} 7^{\mathrm{iii}} \\
& \mathrm{Cs} 3^{\mathrm{iii}}-\mathrm{Cs} 1-\mathrm{O} 7^{\mathrm{iii}} \\
& \mathrm{Cs} 2-\mathrm{Cs} 1-\mathrm{O} 7^{\mathrm{iii}}
\end{aligned}
$$



$$
\begin{aligned}
& \text { Cs } 3^{\mathrm{i}}-\mathrm{Cs} 1-\mathrm{O} 7^{\mathrm{iii}} \\
& \mathrm{Lu} 1^{\mathrm{ii}}-\mathrm{Cs} 1-\mathrm{O} 7^{\mathrm{iii}}
\end{aligned}
$$

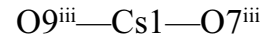

$$
\begin{aligned}
& \mathrm{O} 1^{\mathrm{i}}-\mathrm{Cs} 1-\mathrm{O} 7^{\mathrm{iii}} \\
& \mathrm{O} 6-\mathrm{Cs} 1-\mathrm{O} 7^{\mathrm{iii}} \\
& \mathrm{O} 5^{\mathrm{iii}}-\mathrm{Cs} 1-\mathrm{O} 7^{\mathrm{iii}} \\
& \mathrm{O} 3-\mathrm{Cs} 1-\mathrm{Cs}^{\mathrm{ii}} \\
& \mathrm{O} 4^{\mathrm{i}}-\mathrm{Cs} 1-\mathrm{Cs} 2^{\mathrm{ii}} \\
& \mathrm{O} 1^{\mathrm{ii}}-\mathrm{Cs} 1-\mathrm{Cs} 2^{2 \mathrm{ii}}
\end{aligned}
$$

$39.89(10)$
$94.39(11)$
$147.96(11)$
$123.31(10)$
$146.522(6)$
$105.046(10)$
$53.592(9)$
$108.201(11)$
$35.84(11)$
$54.92(12)$
$86.28(10)$
$119.97(10)$
$96.34(11)$
$150.11(11)$
$148.898(10)$
$101.881(6)$
$58.658(10)$
$77.345(10)$
$54.916(9)$
$113.2(2)$
$111.1(2)$
$109.9(2)$
$111.1(2)$
$108.3(2)$
$102.7(2)$
$57.96(17)$
$55.27(16)$
$130.56(15)$
$126.64(19)$
$99.52(17)$
$48.86(15)$
$148.73(16)$
$70.82(18)$
$61.75(3)$
$38.26(16)$
$118.85(17)$
$74.22(15)$
$130.97(16)$
$75.78(3)$
$133.75(4)$
$72.45(16)$
$70.73(16)$
$74.49(15)$
$176.18(16)$
$56.08(2)$
$110.23(4)$
$51.107(19)$
$140.66(16)$




\begin{tabular}{|c|c|c|c|}
\hline  & $69.12(7)$ & $\mathrm{O} 9-\mathrm{Si} 1-\mathrm{Cs} 2^{\mathrm{vii}}$ & $75.93(16)$ \\
\hline $\mathrm{O} 2^{\mathrm{ii}}-\mathrm{Cs} 1-\mathrm{Cs} 2^{\mathrm{ii}}$ & $96.98(8)$ & $\mathrm{O} 7-\mathrm{Si} 1-\mathrm{Cs} 2^{\mathrm{vii}}$ & $99.98(15)$ \\
\hline $\mathrm{O}^{\mathrm{iv}}-\mathrm{Cs} 1-\mathrm{Cs} 2^{2 \mathrm{i}}$ & $125.63(7)$ & 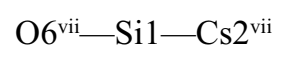 & $35.89(14)$ \\
\hline $\mathrm{Si} 2^{\mathrm{v}}-\mathrm{Cs} 1-\mathrm{Cs} 2^{\mathrm{ii}}$ & $90.16(3)$ & $\mathrm{Cs} 2^{\mathrm{x}}-\mathrm{Si} 1-\mathrm{Cs} 2^{\mathrm{vii}}$ & $117.07(4)$ \\
\hline $\mathrm{O} 2^{\mathrm{vi}}-\mathrm{Cs} 1-\mathrm{Cs} 2^{\mathrm{ii}}$ & $44.23(6)$ & $\mathrm{Cs} 3^{\mathrm{vii}}-\mathrm{Si} 1-\mathrm{Cs} 2^{\mathrm{vii}}$ & $56.30(2)$ \\
\hline $\mathrm{Si} 3^{\mathrm{iii}}-\mathrm{Cs} 1-\mathrm{Cs} 2^{\mathrm{ii}}$ & $69.52(3)$ & $\mathrm{Cs} 3-\mathrm{Si} 1-\mathrm{Cs} 2^{\mathrm{vii}}$ & $165.12(4)$ \\
\hline $\mathrm{Si} 3^{\mathrm{iv}}-\mathrm{Cs} 1-\mathrm{Cs} 2^{\mathrm{ii}}$ & $99.35(3)$ & $\mathrm{Cs} 1^{\mathrm{x}}-\mathrm{Si} 1-\mathrm{Cs} 2^{\mathrm{vii}}$ & $141.46(4)$ \\
\hline $\mathrm{Cs} 3^{\mathrm{iii}}-\mathrm{Cs} 1-\mathrm{Cs} 2^{\mathrm{ii}}$ & $122.524(16)$ & $\mathrm{O} 5-\mathrm{Si} 1-\mathrm{Cs} 1^{\mathrm{vii}}$ & $158.36(17)$ \\
\hline $\mathrm{Cs} 2-\mathrm{Cs} 1-\mathrm{Cs} 2^{\mathrm{ii}}$ & $145.944(14)$ & $\mathrm{O} 9-\mathrm{Si} 1-\mathrm{Cs} 1^{\mathrm{vii}}$ & $85.41(16)$ \\
\hline $\mathrm{Si} 2^{\mathrm{iii}}-\mathrm{Cs} 1-\mathrm{Cs} 2^{\mathrm{ii}}$ & $56.96(2)$ & $\mathrm{O} 7-\mathrm{Si} 1-\mathrm{Cs} 1^{\mathrm{vii}}$ & $49.49(15)$ \\
\hline $\mathrm{Cs} 3^{\mathrm{i}}-\mathrm{Cs} 1-\mathrm{Cs} 2^{\mathrm{ii}}$ & $55.983(11)$ & $\mathrm{O} 6^{\mathrm{vii}}-\mathrm{Si} 1-\mathrm{Cs} 1^{\mathrm{vii}}$ & $70.45(15)$ \\
\hline $\mathrm{Lu} 1^{\mathrm{ii}}-\mathrm{Cs} 1-\mathrm{Cs} 2^{\mathrm{ii}}$ & $82.056(12)$ & $\mathrm{Cs} 2^{\mathrm{x}}-\mathrm{Si} 1-\mathrm{Cs} 1^{\mathrm{vii}}$ & $139.56(4)$ \\
\hline 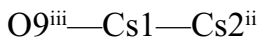 & $107.77(6)$ & $\mathrm{Cs} 3^{\text {vii }}-\mathrm{Si} 1-\mathrm{Cs} 1^{\text {vii }}$ & $101.20(3)$ \\
\hline $\mathrm{O} 1^{\mathrm{i}}-\mathrm{Cs} 1-\mathrm{Cs} 2^{\mathrm{ii}}$ & $95.50(6)$ & $\mathrm{Cs} 3-\mathrm{Si} 1-\mathrm{Cs} 1^{\mathrm{vii}}$ & $123.71(4)$ \\
\hline $\mathrm{O} 6-\mathrm{Cs} 1-\mathrm{Cs} 2^{\mathrm{ii}}$ & $164.48(6)$ & $\mathrm{Cs} 1^{\mathrm{x}}-\mathrm{Si} 1-\mathrm{Cs} 1^{\mathrm{vii}}$ & $105.74(3)$ \\
\hline $\mathrm{O} 5^{\mathrm{iii}}-\mathrm{Cs} 1-\mathrm{Cs} 2^{\mathrm{ii}}$ & $130.80(6)$ & $\mathrm{Cs} 2^{\mathrm{vii}}-\mathrm{Si} 1-\mathrm{Cs} 1^{\mathrm{vii}}$ & $51.995(19)$ \\
\hline $\mathrm{O}^{\mathrm{iii}}-\mathrm{Cs} 1-\mathrm{Cs} 2^{2 \mathrm{ii}}$ & $92.69(6)$ & $\mathrm{O} 5-\mathrm{Si} 1-\mathrm{Cs} 2$ & $81.83(17)$ \\
\hline $\mathrm{O} 3-\mathrm{Cs} 1-\mathrm{Si}^{1 \mathrm{ii}}$ & $146.06(10)$ & O9-Si1-Cs2 & $143.52(16)$ \\
\hline $\mathrm{O} 4{ }^{\mathrm{i}}-\mathrm{Cs} 1-\mathrm{Si} 1^{\mathrm{iii}}$ & $120.06(8)$ & $\mathrm{O} 7-\mathrm{Si} 1-\mathrm{Cs} 2$ & $35.83(15)$ \\
\hline $\mathrm{O} 1^{\mathrm{ii}}-\mathrm{Cs} 1-\mathrm{Si} 1^{\mathrm{iii}}$ & $52.03(8)$ & $\mathrm{O}^{\mathrm{vii}}-\mathrm{Si} 1-\mathrm{Cs} 2$ & $95.27(19)$ \\
\hline $\mathrm{O} 8^{\mathrm{iii}}-\mathrm{Cs} 1-\mathrm{Si} 1^{\mathrm{iii}}$ & $60.28(8)$ & $\mathrm{Cs} 2^{\mathrm{x}}-\mathrm{Si} 1-\mathrm{Cs} 2$ & $128.71(4)$ \\
\hline $\mathrm{O} 2^{\mathrm{ii}}-\mathrm{Cs} 1-\mathrm{Si} 1^{\mathrm{iii}}$ & $70.43(8)$ & $\mathrm{Cs} 3^{\mathrm{vii}}-\mathrm{Si} 1-\mathrm{Cs} 2$ & $165.63(4)$ \\
\hline $\mathrm{O} 7^{\mathrm{iv}}-\mathrm{Cs} 1-\mathrm{Si} 1^{\mathrm{iii}}$ & $117.66(7)$ & $\mathrm{Cs} 3-\mathrm{Si} 1-\mathrm{Cs} 2$ & $53.34(2)$ \\
\hline $\mathrm{Si} 2^{\mathrm{v}}-\mathrm{Cs} 1-\mathrm{Si} 1^{\mathrm{iii}}$ & $97.41(3)$ & $\mathrm{Cs} 1^{\mathrm{x}}-\mathrm{Si} 1-\mathrm{Cs} 2$ & $83.89(3)$ \\
\hline $\mathrm{O} 2^{\mathrm{vi}}-\mathrm{Cs} 1-\mathrm{Si}^{\mathrm{iii}}$ & $106.70(7)$ & $\mathrm{Cs} 2^{\mathrm{vii}}-\mathrm{Si} 1-\mathrm{Cs} 2$ & $114.22(3)$ \\
\hline $\mathrm{Si} 3^{\mathrm{iii}}-\mathrm{Cs} 1-\mathrm{Si} 1^{\mathrm{iii}}$ & $44.42(3)$ & $\mathrm{Cs} 1{ }^{\mathrm{vii}}-\mathrm{Si} 1-\mathrm{Cs} 2$ & $76.55(3)$ \\
\hline $\mathrm{Si}^{\mathrm{iv}}-\mathrm{Cs} 1-\mathrm{Si} 1^{\mathrm{iii}}$ & $143.83(3)$ & $\mathrm{O} 5-\mathrm{Si} 1-\mathrm{Cs} 3^{\mathrm{viii}}$ & $115.92(17)$ \\
\hline $\mathrm{Cs} 3^{\mathrm{iii}}-\mathrm{Cs} 1-\mathrm{Si} 1^{\mathrm{iii}}$ & $62.63(2)$ & $\mathrm{O} 9-\mathrm{Si} 1-\mathrm{Cs} 3^{\text {viii }}$ & $130.79(17)$ \\
\hline $\mathrm{Cs} 2-\mathrm{Cs} 1-\mathrm{Si} 1^{\mathrm{iii}}$ & $54.42(2)$ & $\mathrm{O} 7-\mathrm{Si} 1-\mathrm{Cs} 3^{\text {viii }}$ & $53.48(14)$ \\
\hline $\mathrm{Si} 2^{\mathrm{iii}}-\mathrm{Cs} 1-\mathrm{Si} 1^{\mathrm{iii}}$ & $83.16(3)$ & $\mathrm{O}^{\mathrm{vii}}-\mathrm{Si} 1-\mathrm{Cs} 3^{\mathrm{viii}}$ & $50.38(18)$ \\
\hline $\mathrm{Cs} 3^{\mathrm{i}}-\mathrm{Cs} 1-\mathrm{Si} 1^{\mathrm{iii}}$ & $157.30(2)$ & $\mathrm{Cs} 2^{\mathrm{x}}-\mathrm{Si} 1-\mathrm{Cs} 3^{\mathrm{viii}}$ & $172.92(4)$ \\
\hline $\mathrm{Lu} 1^{\mathrm{ii}}-\mathrm{Cs} 1-\mathrm{Si} 1^{\mathrm{iii}}$ & $50.86(2)$ & $\mathrm{Cs} 3^{\mathrm{vii}}-\mathrm{Si} 1-\mathrm{Cs} 3^{\mathrm{viii}}$ & $118.36(4)$ \\
\hline $\mathrm{O} 9^{\mathrm{iii}}-\mathrm{Cs} 1-\mathrm{Si}^{\mathrm{iii}}$ & $22.07(6)$ & $\mathrm{Cs} 3-\mathrm{Si} 1-\mathrm{Cs} 3^{\mathrm{viii}}$ & $101.51(3)$ \\
\hline $\mathrm{O} 1^{\mathrm{i}}-\mathrm{Cs} 1-\mathrm{Si}^{\mathrm{iii}}$ & $152.41(6)$ & $\mathrm{Cs} 1^{\mathrm{x}}-\mathrm{Si} 1-\mathrm{Cs} 3^{\mathrm{viii}}$ & $127.30(3)$ \\
\hline $\mathrm{O} 6-\mathrm{Cs} 1-\mathrm{Si}^{\mathrm{iii}}$ & $83.18(6)$ & $\mathrm{Cs} 2^{\mathrm{vii}}-\mathrm{Si} 1-\mathrm{Cs} 3^{\mathrm{viii}}$ & $64.81(2)$ \\
\hline $\mathrm{O} 5^{\mathrm{iii}}-\mathrm{Cs} 1-\mathrm{Si} 1^{\mathrm{iii}}$ & $21.97(6)$ & $\mathrm{Cs} 1^{\mathrm{vii}}-\mathrm{Si} 1-\mathrm{Cs} 3^{\mathrm{viii}}$ & $47.326(17)$ \\
\hline $\mathrm{O} 7^{\mathrm{iii}}-\mathrm{Cs} 1-\mathrm{Si} 1^{\mathrm{iii}}$ & $22.66(6)$ & $\mathrm{Cs} 2-\mathrm{Si} 1-\mathrm{Cs} 3^{\mathrm{viii}}$ & $49.844(18)$ \\
\hline $\mathrm{Cs} 22^{\mathrm{ii}}-\mathrm{Cs} 1-\mathrm{Si} 1^{\mathrm{iii}}$ & $111.76(2)$ & $\mathrm{O} 4^{\mathrm{iii}}-\mathrm{Si} 2-\mathrm{O} 2^{\mathrm{iv}}$ & $114.4(2)$ \\
\hline $\mathrm{O} 3-\mathrm{Cs} 1-\mathrm{Si}^{\mathrm{iv}}$ & $67.82(8)$ & $\mathrm{O} 4^{\mathrm{iii}-\mathrm{Si} 2-\mathrm{O} 6}$ & $108.9(2)$ \\
\hline $\mathrm{O} 4-\mathrm{Cs} 1-\mathrm{Si}^{\mathrm{i}}{ }^{\mathrm{iv}}$ & $70.07(9)$ & $\mathrm{O} 2^{\mathrm{iv}}-\mathrm{Si} 2-\mathrm{O} 6$ & $110.9(2)$ \\
\hline $\mathrm{O} 1^{\mathrm{ii}}-\mathrm{Cs} 1-\mathrm{Si}^{\mathrm{iv}}$ & $155.79(8)$ & $\mathrm{O} 4^{\mathrm{iii}-\mathrm{Si} 2-\mathrm{O} 8}$ & $110.2(2)$ \\
\hline $\mathrm{O} 8^{\mathrm{iii}}-\mathrm{Cs} 1-\mathrm{Si}_{1}{ }^{\mathrm{iv}}$ & $130.11(7)$ & $\mathrm{O} 2^{\mathrm{iv}}-\mathrm{Si} 2-\mathrm{O} 8$ & $109.0(2)$ \\
\hline $\mathrm{O} 2^{\mathrm{ii}}-\mathrm{Cs} 1-\mathrm{Si}^{\mathrm{iv}}$ & $99.45(8)$ & $\mathrm{O} 6-\mathrm{Si} 2-\mathrm{O} 8$ & $102.8(2)$ \\
\hline $\mathrm{O}^{\mathrm{iv}}-\mathrm{Cs} 1-\mathrm{Si}^{\mathrm{iv}}$ & $21.25(7)$ & $\mathrm{O} 4^{\mathrm{iii}}-\mathrm{Si} 2-\mathrm{Cs} 1^{\mathrm{xii}}$ & $57.38(15)$ \\
\hline $\mathrm{Si} 2^{\mathrm{v}}-\mathrm{Cs} 1-\mathrm{Si}^{\mathrm{iv}}$ & $89.24(3)$ & $\mathrm{O} 2^{\mathrm{iv}}-\mathrm{Si} 2-\mathrm{Cs} 1^{\mathrm{xiii}}$ & $61.05(17)$ \\
\hline $\mathrm{O} 2^{\mathrm{vi}}-\mathrm{Cs} 1-\mathrm{Si}_{1}{ }^{\mathrm{iv}}$ & $120.01(7)$ & $\mathrm{O} 6-\mathrm{Si} 2-\mathrm{Cs} 1^{\mathrm{xiii}}$ & $109.69(19)$ \\
\hline $\mathrm{Si} 3^{\mathrm{iii}}-\mathrm{Cs} 1-\mathrm{Si} 1^{\mathrm{iv}}$ & $144.25(3)$ & $\mathrm{O} 8-\mathrm{Si} 2-\mathrm{Cs} 1^{\mathrm{xiii}}$ & $147.47(15)$ \\
\hline $\mathrm{Si} 3^{\mathrm{iv}}-\mathrm{Cs} 1-\mathrm{Si}^{\mathrm{iv}}$ & $43.97(3)$ & $\mathrm{O} 4^{\mathrm{iii}}-\mathrm{Si} 2-\mathrm{Cs} 2$ & $87.84(16)$ \\
\hline
\end{tabular}




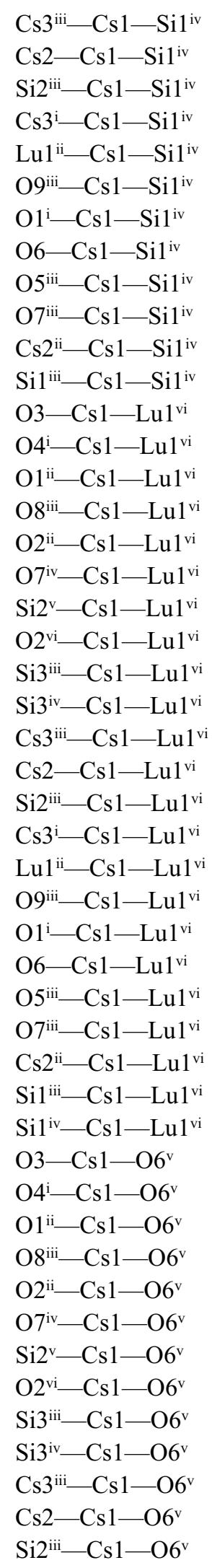

71.69 (2)

$63.91(2)$

$125.74(3)$

89.85 (2)

$127.26(2)$

108.59 (6)

$47.69(6)$

$22.56(6)$

85.35 (6)

$122.62(6)$

$142.25(2)$

$105.74(3)$

$26.72(8)$

$108.40(8)$

$109.36(8)$

$73.39(7)$

$150.75(7)$

$71.56(7)$

$127.96(3)$

$30.44(7)$

$97.11(3)$

$52.34(2)$

82.461 (11)

154.785 (14)

$50.75(2)$

$62.206(10)$

139.577 (14)

149.30 (6)

$66.27(6)$

$108.21(6)$

$126.37(6)$

$111.62(6)$

$59.080(10)$

131.45 (2)

92.64 (2)

$109.67(11)$

36.49 (10)

75.04 (9)

123.67 (9)

$37.56(9)$

116.34 (8)

20.90 (6)

112.95 (9)

$101.46(5)$

$103.82(6)$

$164.12(6)$

83.59 (6)

123.87 (6)

\begin{tabular}{|c|c|}
\hline $\mathrm{O} 2^{\mathrm{iv}}-\mathrm{Si} 2-\mathrm{Cs} 2$ & $157.77(17)$ \\
\hline $\mathrm{O} 6-\mathrm{Si} 2-\mathrm{Cs} 2$ & $58.84(15)$ \\
\hline $\mathrm{O} 8-\mathrm{Si} 2-\mathrm{Cs} 2$ & $59.33(14)$ \\
\hline $\mathrm{Cs} 1{ }^{\mathrm{xiii}-\mathrm{Si}} 2-\mathrm{Cs} 2$ & $139.33(5)$ \\
\hline $\mathrm{O} 4^{\mathrm{iii}}-\mathrm{Si} 2-\mathrm{Cs} 2^{\mathrm{xiii}}$ & $85.86(17)$ \\
\hline $\mathrm{O} 2^{\mathrm{iv}}-\mathrm{Si} 2-\mathrm{Cs} 2^{\mathrm{xiii}}$ & $44.67(15)$ \\
\hline $\mathrm{O} 6-\mathrm{Si} 2-\mathrm{Cs} 2^{\mathrm{xiii}}$ & $155.59(15)$ \\
\hline $\mathrm{O} 8-\mathrm{Si} 2-\mathrm{Cs} 2^{\mathrm{xiii}}$ & $89.49(14)$ \\
\hline $\mathrm{Cs} 1^{\mathrm{xiii}}-\mathrm{Si} 2-\mathrm{Cs} 2^{\mathrm{xiii}}$ & $61.26(3)$ \\
\hline $\mathrm{Cs} 2-\mathrm{Si} 2-\mathrm{Cs} 2^{\mathrm{xiii}}$ & $143.49(4)$ \\
\hline $\mathrm{O} 4^{\mathrm{iii}}-\mathrm{Si} 2-\mathrm{Cs} 1^{\mathrm{x}}$ & $138.82(17)$ \\
\hline $\mathrm{O} 2^{\mathrm{iv}}-\mathrm{Si} 2-\mathrm{Cs} 1^{\mathrm{x}}$ & $63.90(17)$ \\
\hline $\mathrm{O} 6-\mathrm{Si} 2-\mathrm{Cs} 1^{\mathrm{x}}$ & $109.48(18)$ \\
\hline $\mathrm{O} 8-\mathrm{Si} 2-\mathrm{Cs} 1^{\mathrm{x}}$ & $46.25(14)$ \\
\hline $\mathrm{Cs} 1^{\mathrm{xiii}}-\mathrm{Si} 2-\mathrm{Cs} 1^{\mathrm{x}}$ & $120.44(4)$ \\
\hline $\mathrm{Cs} 2-\mathrm{Si} 2-\mathrm{Cs} 1^{\mathrm{x}}$ & $99.38(4)$ \\
\hline $\mathrm{Cs} 2^{\mathrm{xiii}}-\mathrm{Si} 2-\mathrm{Cs} 1^{\mathrm{x}}$ & $64.41(3)$ \\
\hline $\mathrm{O} 4^{\mathrm{iii}}-\mathrm{Si} 2-\mathrm{Cs} 3^{\mathrm{iii}}$ & $40.57(16)$ \\
\hline $\mathrm{O} 2^{\mathrm{iv}}-\mathrm{Si} 2-\mathrm{Cs} 3^{\mathrm{iii}}$ & $119.76(18)$ \\
\hline $\mathrm{O} 6-\mathrm{Si} 2-\mathrm{Cs} 3^{\mathrm{iii}}$ & $69.88(18)$ \\
\hline $\mathrm{O} 8-\mathrm{Si} 2-\mathrm{Cs} 3^{\mathrm{iii}}$ & $130.14(15)$ \\
\hline $\mathrm{Cs} 1^{\mathrm{xii}}-\mathrm{Si} 2-\mathrm{Cs} 3^{\mathrm{iii}}$ & $62.82(3)$ \\
\hline $\mathrm{Cs} 2-\mathrm{Si} 2-\mathrm{Cs} 3^{\mathrm{iii}}$ & $77.16(3)$ \\
\hline $\mathrm{Cs} 2^{\mathrm{xiii}}-\mathrm{Si} 2-\mathrm{Cs} 3^{\mathrm{iii}}$ & $117.73(4)$ \\
\hline $\mathrm{Cs} 1^{\mathrm{x}}-\mathrm{Si} 2-\mathrm{Cs} 3^{\mathrm{iii}}$ & $176.34(4)$ \\
\hline 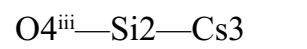 & $146.46(17)$ \\
\hline $\mathrm{O} 2^{\mathrm{iv}}-\mathrm{Si} 2-\mathrm{Cs} 3$ & $98.88(17)$ \\
\hline $\mathrm{O} 6-\mathrm{Si} 2-\mathrm{Cs} 3$ & $59.64(19)$ \\
\hline $\mathrm{O} 8-\mathrm{Si} 2-\mathrm{Cs} 3$ & $51.01(13)$ \\
\hline $\mathrm{Cs} 1^{\mathrm{xiii}}-\mathrm{Si} 2-\mathrm{Cs} 3$ & $153.99(4)$ \\
\hline $\mathrm{Cs} 2-\mathrm{Si} 2-\mathrm{Cs} 3$ & $58.92(2)$ \\
\hline $\mathrm{Cs} 2^{\mathrm{xii}}-\mathrm{Si} 2-\mathrm{Cs} 3$ & $117.51(4)$ \\
\hline $\mathrm{Cs} 11^{\mathrm{x}}-\mathrm{Si} 2-\mathrm{Cs} 3$ & $53.25(2)$ \\
\hline $\mathrm{Cs} 33^{\mathrm{iii}}-\mathrm{Si} 2-\mathrm{Cs} 3$ & $124.76(4)$ \\
\hline  & $76.58(17)$ \\
\hline $\mathrm{O} 2^{\mathrm{iv}}-\mathrm{Si} 2-\mathrm{Cs} 1$ & $137.47(17)$ \\
\hline $\mathrm{O} 6-\mathrm{Si} 2-\mathrm{Cs} 1$ & $34.26(15)$ \\
\hline $\mathrm{O} 8-\mathrm{Si} 2-\mathrm{Cs} 1$ & $104.15(15)$ \\
\hline $\mathrm{Cs} 1^{\mathrm{xiii}}-\mathrm{Si} 2-\mathrm{Cs} 1$ & $101.58(3)$ \\
\hline $\mathrm{Cs} 2-\mathrm{Si} 2-\mathrm{Cs} 1$ & $45.200(19)$ \\
\hline $\mathrm{Cs} 2^{\mathrm{xiii}}-\mathrm{Si} 2-\mathrm{Cs} 1$ & $160.60(4)$ \\
\hline $\mathrm{Cs} 1{ }^{\mathrm{x}}-\mathrm{Si} 2-\mathrm{Cs} 1$ & $134.92(4)$ \\
\hline 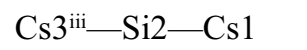 & $42.877(16)$ \\
\hline $\mathrm{Cs} 3-\mathrm{Si} 2-\mathrm{Cs} 1$ & $81.89(2)$ \\
\hline $\mathrm{O} 4^{\mathrm{iii}}-\mathrm{Si} 2-\mathrm{Cs} 3^{\mathrm{xiii}}$ & $67.32(16)$ \\
\hline $\mathrm{O} 2^{\mathrm{iv}}-\mathrm{Si} 2-\mathrm{Cs} 3^{\mathrm{xiii}}$ & $89.29(16)$ \\
\hline $\mathrm{O} 6-\mathrm{Si} 2-\mathrm{Cs} 3^{\mathrm{xiii}}$ & $158.25(15)$ \\
\hline $\mathrm{O} 8-\mathrm{Si} 2-\mathrm{Cs} 3^{\mathrm{xiii}}$ & $61.41(14)$ \\
\hline
\end{tabular}




$$
\begin{aligned}
& \mathrm{Cs} 3^{\mathrm{i}}-\mathrm{Cs} 1-\mathrm{O}^{\mathrm{v}} \\
& \mathrm{Lu} 1^{\mathrm{ii}}-\mathrm{Cs} 1-\mathrm{O}^{\mathrm{v}} \\
& \mathrm{O} 9^{\mathrm{iii}}-\mathrm{Cs} 1-\mathrm{O}^{\mathrm{v}} \\
& \mathrm{O} 1^{\mathrm{i}}-\mathrm{Cs} 1-\mathrm{O}^{\mathrm{v}} \\
& \mathrm{O} 6-\mathrm{Cs} 1-\mathrm{O}^{\mathrm{v}} \\
& \mathrm{O} 5^{\mathrm{iii}}-\mathrm{Cs} 1-\mathrm{O}^{\mathrm{v}} \\
& \mathrm{O} 7^{\mathrm{iii}}-\mathrm{Cs} 1-\mathrm{O}^{\mathrm{v}} \\
& \mathrm{Cs} 22^{\mathrm{ii}}-\mathrm{Cs} 1-\mathrm{O}^{\mathrm{v}}
\end{aligned}
$$

\begin{tabular}{|c|c|c|}
\hline $54.80(6)$ & $\mathrm{Cs} 1^{\mathrm{xiii}}-\mathrm{Si} 2-\mathrm{Cs} 3^{\mathrm{xiii}}$ & $86.70(3)$ \\
\hline $54.89(5)$ & $\mathrm{Cs} 2-\mathrm{Si} 2-\mathrm{Cs} 3^{\mathrm{xiii}}$ & $99.41(3)$ \\
\hline $82.45(8)$ & $\mathrm{Cs} 2^{\mathrm{xiii}}-\mathrm{Si} 2-\mathrm{Cs} 3^{\mathrm{xiii}}$ & $45.395(17)$ \\
\hline $80.95(7)$ & $\mathrm{Cs} 1^{x}-\mathrm{Si} 2-\mathrm{Cs} 3^{\mathrm{xiii}}$ & $71.50(2)$ \\
\hline $112.32(11)$ & $\mathrm{Cs} 3^{\mathrm{iii}}-\mathrm{Si} 2-\mathrm{Cs} 3^{\mathrm{xiii}}$ & $107.68(3)$ \\
\hline $118.59(9)$ & $\mathrm{Cs} 3-\mathrm{Si} 2-\mathrm{Cs} 3^{\mathrm{xiii}}$ & $110.86(3)$ \\
\hline $109.72(8)$ & $\mathrm{Cs} 1-\mathrm{Si} 2-\mathrm{Cs} 3^{\mathrm{xiii}}$ & $130.50(3)$ \\
\hline $69.27(6)$ & $\mathrm{O} 3^{\mathrm{vii}}-\mathrm{Si} 3-\mathrm{O} 1^{\text {xiii }}$ & $114.7(2)$ \\
\hline $104.15(6)$ & $\mathrm{O}^{\mathrm{vii}}-\mathrm{Si} 3-\mathrm{O} 7$ & $108.3(2)$ \\
\hline $105.97(6)$ & $\mathrm{O} 1^{\mathrm{xii}-\mathrm{Si} 3-\mathrm{O} 7}$ & $110.7(2)$ \\
\hline $113.42(6)$ & $\mathrm{O}^{\mathrm{vii}}-\mathrm{Si} 3-\mathrm{O} 8$ & $109.6(2)$ \\
\hline $60.35(11)$ & $\mathrm{O} 1^{\mathrm{xii}}-\mathrm{Si} 3-\mathrm{O} 8$ & $110.1(2)$ \\
\hline $116.46(11)$ & $\mathrm{O} 7-\mathrm{Si} 3-\mathrm{O} 8$ & $102.7(2)$ \\
\hline $129.40(12)$ & $\mathrm{O} 3^{\mathrm{vii}}-\mathrm{Si} 3-\mathrm{Cs} 1^{\mathrm{x}}$ & $153.15(18)$ \\
\hline $162.29(11)$ & $\mathrm{O} 1^{\mathrm{xiii}}-\mathrm{Si} 3-\mathrm{Cs} 1^{\mathrm{x}}$ & $57.13(16)$ \\
\hline $134.84(10)$ & $\mathrm{O} 7-\mathrm{Si} 3-\mathrm{Cs}^{\mathrm{x}}$ & $98.21(15)$ \\
\hline $48.68(10)$ & $\mathrm{O} 8-\mathrm{Si} 3-\mathrm{Cs} 1^{\mathrm{x}}$ & $58.73(14)$ \\
\hline $100.65(11)$ & $\mathrm{O} 3^{\mathrm{vii}}-\mathrm{Si} 3-\mathrm{Cs} 1^{\mathrm{vii}}$ & $51.18(16)$ \\
\hline $50.71(10)$ & $\mathrm{O} 1^{\mathrm{xiii}}-\mathrm{Si} 3-\mathrm{Cs} 1^{\mathrm{vii}}$ & $94.80(16)$ \\
\hline $85.47(11)$ & $\mathrm{O} 7-\mathrm{Si} 3-\mathrm{Cs} 1^{\mathrm{vii}}$ & $73.12(15)$ \\
\hline $88.54(10)$ & $\mathrm{O} 8-\mathrm{Si} 3-\mathrm{Cs} 1^{\mathrm{vii}}$ & $154.24(16)$ \\
\hline $142.42(12)$ & $\mathrm{Cs} 1^{\mathrm{x}}-\mathrm{Si} 3-\mathrm{Cs} 1^{\mathrm{vii}}$ & $146.38(5)$ \\
\hline $113.07(10)$ & 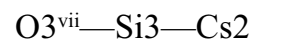 & $81.85(18)$ \\
\hline $96.27(10)$ & $\mathrm{O} 1^{\mathrm{xiii}}-\mathrm{Si} 3-\mathrm{Cs} 2$ & $163.35(17)$ \\
\hline $48.07(10)$ & $\mathrm{O} 7-\mathrm{Si} 3-\mathrm{Cs} 2$ & $62.53(15)$ \\
\hline $99.82(11)$ & $\mathrm{O} 8-\mathrm{Si} 3-\mathrm{Cs} 2$ & $59.86(14)$ \\
\hline $168.59(9)$ & $\mathrm{Cs} 1{ }^{\mathrm{x}}-\mathrm{Si} 3-\mathrm{Cs} 2$ & $107.41(4)$ \\
\hline $115.18(8)$ & $\mathrm{Cs} 1{ }^{\mathrm{vii}}-\mathrm{Si} 3-\mathrm{Cs} 2$ & $97.32(4)$ \\
\hline $74.72(8)$ & $\mathrm{O} 3^{\mathrm{vii}}-\mathrm{Si} 3-\mathrm{Cs} 3^{\mathrm{xiii}}$ & $64.63(17)$ \\
\hline $27.25(7)$ & $\mathrm{O} 1^{\mathrm{xiii}}-\mathrm{Si} 3-\mathrm{Cs} 3^{\mathrm{xiii}}$ & $50.25(15)$ \\
\hline $81.86(8)$ & $\mathrm{O} 7-\mathrm{Si} 3-\mathrm{Cs} 3^{\mathrm{xiii}}$ & $131.83(16)$ \\
\hline $27.12(7)$ & $\mathrm{O} 8-\mathrm{Si} 3-\mathrm{Cs} 3^{\mathrm{xiii}}$ & $124.96(15)$ \\
\hline $141.91(9)$ & $\mathrm{Cs} 1^{x}-\mathrm{Si} 3-\mathrm{Cs} 3^{\mathrm{xiii}}$ & $100.79(4)$ \\
\hline $125.57(8)$ & 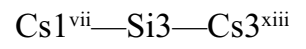 & $66.63(3)$ \\
\hline $26.81(7)$ & $\mathrm{Cs} 2-\mathrm{Si} 3-\mathrm{Cs} 3^{\mathrm{xiii}}$ & $146.01(5)$ \\
\hline $27.00(7)$ & $\mathrm{O}^{\mathrm{vii}}-\mathrm{Si} 3-\mathrm{Cs} 3$ & $136.15(18)$ \\
\hline $74.91(8)$ & $\mathrm{O} 1^{\mathrm{xiii}}-\mathrm{Si} 3-\mathrm{Cs} 3$ & $107.39(16)$ \\
\hline $74.19(7)$ & $\mathrm{O} 7-\mathrm{Si} 3-\mathrm{Cs} 3$ & $65.40(15)$ \\
\hline $49.50(3)$ & $\mathrm{O} 8-\mathrm{Si} 3-\mathrm{Cs} 3$ & $40.97(13)$ \\
\hline $80.29(8)$ & $\mathrm{Cs} 1{ }^{\mathrm{x}}-\mathrm{Si} 3-\mathrm{Cs} 3$ & $52.75(2)$ \\
\hline $25.30(8)$ & $\mathrm{Cs} 1{ }^{\mathrm{vii}}-\mathrm{Si} 3-\mathrm{Cs} 3$ & $137.60(4)$ \\
\hline $108.13(9)$ & $\mathrm{Cs} 2-\mathrm{Si} 3-\mathrm{Cs} 3$ & $56.09(2)$ \\
\hline $112.01(7)$ & $\mathrm{Cs} 3^{\mathrm{xiii}}-\mathrm{Si} 3-\mathrm{Cs} 3$ & $153.02(4)$ \\
\hline $25.42(8)$ & $\mathrm{O} 3^{\mathrm{vii}}-\mathrm{Si} 3-\mathrm{Cs} 2^{\mathrm{xiii}}$ & $92.04(17)$ \\
\hline $107.81(8)$ & $\mathrm{O} 1^{\mathrm{xiii}}-\mathrm{Si} 3-\mathrm{Cs} 2^{\mathrm{xiii}}$ & $56.69(15)$ \\
\hline $98.82(3)$ & $\mathrm{O} 7-\mathrm{Si} 3-\mathrm{Cs} 2^{\mathrm{xiii}}$ & $159.60(15)$ \\
\hline $100.32(3)$ & $\mathrm{O} 8-\mathrm{Si} 3-\mathrm{Cs} 2^{\mathrm{xiii}}$ & $70.95(14)$ \\
\hline $152.44(10)$ & $\mathrm{Cs} 1^{\mathrm{x}}-\mathrm{Si} 3-\mathrm{Cs} 2^{\mathrm{xiii}}$ & $61.71(2)$ \\
\hline
\end{tabular}

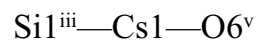

$$
\begin{aligned}
& \mathrm{Si} 1^{\mathrm{iv}}-\mathrm{Cs} 1-\mathrm{O}^{\mathrm{v}} \\
& \mathrm{Lu} 1^{\mathrm{vi}}-\mathrm{Cs} 1-\mathrm{O}^{\mathrm{v}}
\end{aligned}
$$

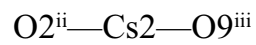

$$
\begin{aligned}
& \mathrm{O} 22^{\mathrm{ii}}-\mathrm{Cs} 2-\mathrm{O} 6 \\
& \mathrm{O} 9 \mathrm{iii}-\mathrm{Cs} 2-\mathrm{O} 6 \\
& \mathrm{O} 22^{\mathrm{ii}}-\mathrm{Cs} 2-\mathrm{O} 8 \\
& \mathrm{O} 9^{\mathrm{iii}-\mathrm{Cs} 2-\mathrm{O} 8} \\
& \mathrm{O} 6-\mathrm{Cs} 2-\mathrm{O} 8 \\
& \mathrm{O} 2^{\mathrm{ii}}-\mathrm{Cs} 2-\mathrm{O} 5^{\mathrm{iii}} \\
& \mathrm{O} 9^{\mathrm{iii}}-\mathrm{Cs} 2-\mathrm{O} 5^{\mathrm{iii}} \\
& \mathrm{O} 6-\mathrm{Cs} 2-\mathrm{O} 5^{\mathrm{iii}} \\
& \mathrm{O} 8-\mathrm{Cs} 2-\mathrm{O}^{\mathrm{iii}} \\
& \mathrm{O} 22^{\mathrm{ii}}-\mathrm{Cs} 2-\mathrm{O} 7 \\
& \mathrm{O} 9 \text { iii-Cs2-O7 } \\
& \text { O6- Cs2-O7 } \\
& \mathrm{O} 8-\mathrm{Cs} 2-\mathrm{O} 7
\end{aligned}
$$



$$
\begin{aligned}
& \mathrm{O} 22^{\mathrm{ii}}-\mathrm{Cs} 2-\mathrm{Si} 3 \\
& \text { O9 }{ }^{\mathrm{iii}-\mathrm{Cs} 2-\mathrm{Si} 3} \\
& \mathrm{O} 6-\mathrm{Cs} 2-\mathrm{Si} 3 \\
& \mathrm{O} 8-\mathrm{Cs} 2-\mathrm{Si} 3 \\
& \mathrm{O} 5 \text { iii- } \mathrm{Cs} 2-\mathrm{Si} 3 \\
& \mathrm{O} 7-\mathrm{Cs} 2-\mathrm{Si} 3 \\
& \mathrm{O} 2{ }^{\mathrm{ii}}-\mathrm{Cs} 2-\mathrm{Si} 2 \\
& \mathrm{O} 9^{\mathrm{iii}}-\mathrm{Cs} 2-\mathrm{Si} 2 \\
& \text { O6- Cs2-Si2 } \\
& \mathrm{O} 8-\mathrm{Cs} 2-\mathrm{Si} 2 \\
& \mathrm{O} 5{ }^{\mathrm{iii}}-\mathrm{Cs} 2-\mathrm{Si} 2 \\
& \mathrm{O} 7-\mathrm{Cs} 2-\mathrm{Si} 2 \\
& \mathrm{Si} 3-\mathrm{Cs} 2-\mathrm{Si} 2 \\
& \mathrm{O} 2^{\mathrm{ii}}-\mathrm{Cs} 2-\mathrm{Si}^{\mathrm{iii}} \\
& \text { O9ii- } \mathrm{Cs} 2-\mathrm{Si}^{\mathrm{iii}} \\
& \text { O6- Cs2-Si } 1^{i i i} \\
& \mathrm{O} 8-\mathrm{Cs} 2-\mathrm{Si}^{1}{ }^{\mathrm{iii}} \\
& \mathrm{O} 5^{\mathrm{iii}}-\mathrm{Cs} 2-\mathrm{Si}^{\mathrm{iii}} \\
& \mathrm{O} 7-\mathrm{Cs} 2-\mathrm{Si}^{1}{ }^{\mathrm{iii}} \\
& \mathrm{Si} 3-\mathrm{Cs} 2-\mathrm{Si} 1^{\mathrm{iii}} \\
& \mathrm{Si} 2-\mathrm{Cs} 2-\mathrm{Si}^{\mathrm{iii}} \\
& \mathrm{O} 2^{\mathrm{ii}}-\mathrm{Cs} 2-\mathrm{O}^{\mathrm{vii}}
\end{aligned}
$$






$92.16(10)$
$81.52(9)$
$44.34(10)$
$58.02(10)$
$43.55(10)$
$24.87(7)$
$54.92(7)$
$73.99(7)$
$52.35(9)$
$71.11(8)$
$71.60(8)$
$118.44(7)$
$71.37(8)$
$165.18(7)$
$138.07(3)$
$91.75(3)$
$69.50(2)$
$123.97(7)$
$76.22(10)$
$122.55(10)$
$102.09(11)$
$96.35(9)$
$172.44(10)$
$79.40(9)$
$99.88(6)$
$111.86(7)$
$147.70(7)$
$122.49(9)$
$110.92(6)$
$87.93(8)$
$49.43(7)$
$151.89(7)$
$109.11(7)$
$75.88(8)$
$67.09(7)$
$81.86(3)$
$125.72(3)$
$60.02(2)$
$70.81(7)$
$120.123(14)$
$97.01(7)$
$110.12(8)$
$167.48(8)$
$61.02(9)$
$56.18(7)$
$141.80(8)$
$68.79(7)$
$72.48(2)$

\begin{tabular}{|c|c|}
\hline  & $121.05(4)$ \\
\hline $\mathrm{Cs} 2-\mathrm{Si} 3-\mathrm{Cs} 2^{\mathrm{xiii}}$ & $124.31(4)$ \\
\hline $\mathrm{Cs} 3^{\mathrm{xiii}}-\mathrm{Si} 3-\mathrm{Cs} 2^{\mathrm{xiii}}$ & $55.42(2)$ \\
\hline $\mathrm{Cs} 3-\mathrm{Si} 3-\mathrm{Cs} 2^{\mathrm{xiii}}$ & $101.28(3)$ \\
\hline $\mathrm{O} 3^{\text {vii }}-\mathrm{Si} 3-\mathrm{Cs} 3^{\text {viii }}$ & $62.66(17)$ \\
\hline $\mathrm{O} 1^{\mathrm{xiii}}-\mathrm{Si} 3-\mathrm{Cs} 3^{\mathrm{viii}}$ & $136.69(16)$ \\
\hline $\mathrm{O} 7-\mathrm{Si} 3-\mathrm{Cs} 3^{\text {viii }}$ & $46.17(14)$ \\
\hline $\mathrm{O} 8-\mathrm{Si} 3-\mathrm{Cs} 3^{\text {viii }}$ & $110.86(15)$ \\
\hline $\mathrm{Cs} 1^{x}-\mathrm{Si} 3-\mathrm{Cs} 3^{\text {viii }}$ & $142.47(4)$ \\
\hline $\mathrm{Cs} 1^{\mathrm{vii}}-\mathrm{Si} 3-\mathrm{Cs} 3^{\mathrm{viii}}$ & 47.709 (19) \\
\hline $\mathrm{Cs} 2-\mathrm{Si} 3-\mathrm{Cs} 3^{\mathrm{viii}}$ & $51.00(2)$ \\
\hline $\mathrm{Cs} 3^{\text {xiii }}-\mathrm{Si} 3-\mathrm{Cs} 3^{\text {viii }}$ & $112.21(3)$ \\
\hline $\mathrm{Cs} 3-\mathrm{Si} 3-\mathrm{Cs} 3^{\text {viii }}$ & $94.54(3)$ \\
\hline $\mathrm{Cs} 2^{\mathrm{xiii}}-\mathrm{Si} 3-\mathrm{Cs} 3^{\mathrm{viii}}$ & $154.16(4)$ \\
\hline $\mathrm{O} 3^{\mathrm{vii}}-\mathrm{Si} 3-\mathrm{Cs} 2^{\mathrm{x}}$ & $160.88(17)$ \\
\hline $\mathrm{O} 1^{\mathrm{xiii}}-\mathrm{Si} 3-\mathrm{Cs} 2^{\mathrm{x}}$ & $60.62(16)$ \\
\hline $\mathrm{O} 7-\mathrm{Si} 3-\mathrm{Cs} 2^{\mathrm{x}}$ & $61.07(14)$ \\
\hline $\mathrm{O} 8-\mathrm{Si} 3-\mathrm{Cs} 2^{\mathrm{x}}$ & $88.89(14)$ \\
\hline $\mathrm{Cs} 1^{\mathrm{x}}-\mathrm{Si} 3-\mathrm{Cs} 2^{\mathrm{x}}$ & $42.697(19)$ \\
\hline $\mathrm{Cs} 1^{\mathrm{vii}}-\mathrm{Si} 3-\mathrm{Cs} 2^{\mathrm{x}}$ & $109.74(3)$ \\
\hline $\mathrm{Cs} 2-\mathrm{Si} 3-\mathrm{Cs} 2^{\mathrm{x}}$ & $104.30(3)$ \\
\hline $\mathrm{Cs} 3^{\mathrm{xiii}}-\mathrm{Si} 3-\mathrm{Cs} 2^{\mathrm{x}}$ & $109.29(3)$ \\
\hline $\mathrm{Cs} 3-\mathrm{Si} 3-\mathrm{Cs} 2^{\mathrm{x}}$ & $56.810(18)$ \\
\hline $\mathrm{Cs} 2^{\mathrm{xiii}}-\mathrm{Si} 3-\mathrm{Cs} 2^{\mathrm{x}}$ & $98.93(3)$ \\
\hline $\mathrm{Cs} 3^{\mathrm{viii}}-\mathrm{Si} 3-\mathrm{Cs} 2^{\mathrm{x}}$ & $106.84(3)$ \\
\hline $\mathrm{Si} 3^{\mathrm{v}}-\mathrm{O} 1-\mathrm{Lu} 1$ & $134.3(2)$ \\
\hline $\mathrm{Si} 3^{\mathrm{v}}-\mathrm{O} 1-\mathrm{Cs} 3$ & $104.8(2)$ \\
\hline $\mathrm{Lu} 1-\mathrm{O} 1-\mathrm{Cs} 3$ & $91.04(13)$ \\
\hline $\mathrm{Si}^{2}{ }^{\mathrm{v}}-\mathrm{O} 1-\mathrm{Cs}^{1}{ }^{\mathrm{xiv}}$ & $96.38(18)$ \\
\hline $\mathrm{Lu} 1-\mathrm{O} 1-\mathrm{Cs}^{1 \mathrm{xiv}}$ & $97.95(14)$ \\
\hline $\mathrm{Cs} 3-\mathrm{O} 1-\mathrm{Cs}^{1 \mathrm{xiv}}$ & $140.01(15)$ \\
\hline $\mathrm{Si} 3$ - $-\mathrm{O} 1-\mathrm{Cs} 2$ & $102.74(17)$ \\
\hline $\mathrm{Lu} 1-\mathrm{O} 1-\mathrm{Cs} 2$ & $122.93(14)$ \\
\hline $\mathrm{Cs} 3-\mathrm{O} 1-\mathrm{Cs} 2$ & $68.04(8)$ \\
\hline $\mathrm{Cs} 1^{\mathrm{xiv}}-\mathrm{O} 1-\mathrm{Cs} 2$ & $74.50(9)$ \\
\hline $\mathrm{Si} 3^{\mathrm{v}}-\mathrm{O} 1-\mathrm{Cs} 1^{\mathrm{ix}}$ & $61.90(14)$ \\
\hline $\mathrm{Lu} 1-\mathrm{O} 1-\mathrm{Cs} 1^{\mathrm{ix}}$ & $86.80(12)$ \\
\hline $\mathrm{Cs} 3-\mathrm{O} 1-\mathrm{Cs} 1^{\mathrm{ix}}$ & $67.65(8)$ \\
\hline $\mathrm{Cs} 1^{\mathrm{xiv}}-\mathrm{O} 1-\mathrm{Cs} 1^{\mathrm{ix}}$ & $151.28(13)$ \\
\hline $\mathrm{Cs} 2-\mathrm{O} 1-\mathrm{Cs} 1^{\mathrm{ix}}$ & $126.11(11)$ \\
\hline $\mathrm{Si} 3^{\mathrm{v}}-\mathrm{O} 1-\mathrm{Cs} 2^{2^{\mathrm{xiv}}}$ & $102.96(17)$ \\
\hline $\mathrm{Lu} 1-\mathrm{O} 1-\mathrm{Cs} 2^{\mathrm{xiv}}$ & $57.87(9)$ \\
\hline $\mathrm{Cs} 3-\mathrm{O} 1-\mathrm{Cs} 2^{2^{\mathrm{iv}}}$ & $148.00(12)$ \\
\hline $\mathrm{Cs} 1^{\mathrm{xiv}}-\mathrm{O} 1-\mathrm{Cs} 2^{\mathrm{xiv}}$ & $49.83(6)$ \\
\hline $\mathrm{Cs} 2-\mathrm{O} 1-\mathrm{Cs} 2^{\mathrm{xiv}}$ & $120.32(10)$ \\
\hline $\mathrm{Cs} 1^{\mathrm{ix}}-\mathrm{O} 1-\mathrm{Cs} 2^{\mathrm{xiv}}$ & $113.54(9)$ \\
\hline $\mathrm{Si}^{\mathrm{v}}-\mathrm{O} 1-\mathrm{Cs} 3^{\mathrm{v}}$ & $55.25(14)$ \\
\hline $\mathrm{Lu} 1-\mathrm{O} 1-\mathrm{Cs} 3^{\mathrm{v}}$ & $114.01(13)$ \\
\hline
\end{tabular}




\begin{tabular}{|c|c|}
\hline $\mathrm{Si} 2-\mathrm{Cs} 2-\mathrm{Cs} 3$ & $66.93(2)$ \\
\hline 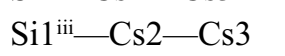 & $167.22(3)$ \\
\hline $\mathrm{O} 3^{\mathrm{vii}}-\mathrm{Cs} 2-\mathrm{Cs} 3$ & $96.75(7)$ \\
\hline $\mathrm{Cs} 1-\mathrm{Cs} 2-\mathrm{Cs} 3$ & $110.429(14)$ \\
\hline $\mathrm{O} 1-\mathrm{Cs} 2-\mathrm{Cs} 3$ & $44.97(6)$ \\
\hline 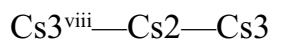 & $125.956(16)$ \\
\hline $\mathrm{O} 2^{\mathrm{ii}}-\mathrm{Cs} 2-\mathrm{Si}^{\mathrm{v}}$ & $22.24(8)$ \\
\hline 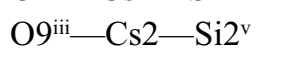 & $82.51(8)$ \\
\hline $\mathrm{O} 6-\mathrm{Cs} 2-\mathrm{Si} 2^{\mathrm{v}}$ & $101.92(7)$ \\
\hline $\mathrm{O} 8-\mathrm{Cs} 2-\mathrm{Si}^{2}{ }^{\mathrm{V}}$ & $140.84(7)$ \\
\hline 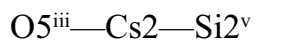 & $117.87(8)$ \\
\hline $\mathrm{O} 7-\mathrm{Cs} 2-\mathrm{Si} 2^{\mathrm{v}}$ & $138.99(8)$ \\
\hline $\mathrm{Si} 3-\mathrm{Cs} 2-\mathrm{Si}^{\mathrm{v}}$ & $159.96(3)$ \\
\hline $\mathrm{Si} 2-\mathrm{Cs} 2-\mathrm{Si}_{2}^{\mathrm{v}}$ & $128.69(3)$ \\
\hline 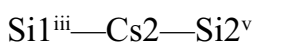 & $100.95(3)$ \\
\hline $\mathrm{O}^{\mathrm{vii}}-\mathrm{Cs} 2-\mathrm{Si}^{\mathrm{v}}$ & $174.67(7)$ \\
\hline $\mathrm{Cs} 1-\mathrm{Cs} 2-\mathrm{Si}^{\mathrm{v}}$ & $54.31(2)$ \\
\hline $\mathrm{O} 1-\mathrm{Cs} 2-\mathrm{Si}_{2}^{\mathrm{v}}$ & $61.06(6)$ \\
\hline $\mathrm{Cs} 3^{\mathrm{viii}}-\mathrm{Cs} 2-\mathrm{Si} 2^{\mathrm{v}}$ & $105.34(2)$ \\
\hline $\mathrm{Cs} 3-\mathrm{Cs} 2-\mathrm{Si} 2^{\mathrm{v}}$ & $88.51(2)$ \\
\hline $\mathrm{O} 2^{\mathrm{ii}}-\mathrm{Cs} 2-\mathrm{O} 4^{\mathrm{iii}}$ & $136.35(11)$ \\
\hline  & $101.65(10)$ \\
\hline $\mathrm{O} 6-\mathrm{Cs} 2-\mathrm{O} 4^{\mathrm{iii}}$ & $42.12(9)$ \\
\hline $\mathrm{O} 8-\mathrm{Cs} 2-\mathrm{O} 4^{\mathrm{iii}}$ & $42.65(9)$ \\
\hline 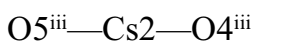 & $50.93(9)$ \\
\hline $\mathrm{O} 7-\mathrm{Cs} 2-\mathrm{O} 4^{\mathrm{iii}}$ & $80.39(10)$ \\
\hline $\mathrm{Si} 3-\mathrm{Cs} 2-\mathrm{O} 4^{\mathrm{iii}}$ & $53.29(7)$ \\
\hline $\mathrm{Si} 2-\mathrm{Cs} 2-\mathrm{O} 4^{\mathrm{iii}}$ & $24.01(6)$ \\
\hline $\mathrm{Si} 1^{\mathrm{iii}}-\mathrm{Cs} 2-\mathrm{O} 4^{\mathrm{iii}}$ & $76.35(6)$ \\
\hline $\mathrm{O} 3^{\mathrm{vii}}-\mathrm{Cs} 2-\mathrm{O} 4^{\mathrm{iii}}$ & $45.57(9)$ \\
\hline $\mathrm{Cs} 1-\mathrm{Cs} 2-\mathrm{O} 4^{\mathrm{iii}}$ & $84.84(6)$ \\
\hline $\mathrm{O} 1-\mathrm{Cs} 2-\mathrm{O} 4^{\mathrm{iii}}$ & $135.67(8)$ \\
\hline $\mathrm{Cs} 3^{\mathrm{viii}}-\mathrm{Cs} 2-\mathrm{O} 4^{\mathrm{iii}}$ & $110.60(6)$ \\
\hline $\mathrm{Cs} 3-\mathrm{Cs} 2-\mathrm{O} 4^{\mathrm{iii}}$ & $90.87(6)$ \\
\hline $\mathrm{Si} 2^{\mathrm{v}}-\mathrm{Cs} 2-\mathrm{O} 4^{\mathrm{iii}}$ & $135.59(7)$ \\
\hline $\mathrm{O} 2^{\mathrm{ii}}-\mathrm{Cs} 2-\mathrm{O} 4^{\mathrm{i}}$ & $40.92(10)$ \\
\hline $\mathrm{O} 9^{\mathrm{iii}}-\mathrm{Cs} 2-\mathrm{O} 4^{\mathrm{i}}$ & $96.37(10)$ \\
\hline $\mathrm{O} 6-\mathrm{Cs} 2-\mathrm{O} 4^{\mathrm{i}}$ & $79.08(9)$ \\
\hline $\mathrm{O} 8-\mathrm{Cs} 2-\mathrm{O} 4^{\mathrm{i}}$ & $121.40(9)$ \\
\hline $\mathrm{O} 5^{\mathrm{iii}}-\mathrm{Cs} 2-\mathrm{O} 4^{\mathrm{i}}$ & $115.22(9)$ \\
\hline $\mathrm{O} 7-\mathrm{Cs} 2-\mathrm{O} 4^{\mathrm{i}}$ & $143.93(9)$ \\
\hline $\mathrm{Si} 3-\mathrm{Cs} 2-\mathrm{O} 4^{\mathrm{i}}$ & $147.41(6)$ \\
\hline $\mathrm{Si} 2-\mathrm{Cs} 2-\mathrm{O} 4^{\mathrm{i}}$ & $105.88(6)$ \\
\hline 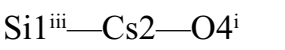 & $107.58(6)$ \\
\hline $\mathrm{O} 3^{\mathrm{vii}}-\mathrm{Cs} 2-\mathrm{O}^{\mathrm{i}}$ & $159.99(9)$ \\
\hline $\mathrm{Cs} 1-\mathrm{Cs} 2-\mathrm{O} 4^{\mathrm{i}}$ & $43.99(6)$ \\
\hline $\mathrm{O} 1-\mathrm{Cs} 2-\mathrm{O} 4^{\mathrm{i}}$ & $66.98(8)$ \\
\hline $\mathrm{Cs} 3^{\text {viii-Cs} 2-O 4^{\mathrm{i}}}$ & $127.87(6)$ \\
\hline
\end{tabular}

\begin{tabular}{|c|c|}
\hline $\mathrm{Cs} 3-\mathrm{O} 1-\mathrm{Cs} 3^{\mathrm{v}}$ & $154.67(12)$ \\
\hline $\mathrm{Cs} 1^{\mathrm{xiv}}-\mathrm{O} 1-\mathrm{Cs} 3^{\mathrm{v}}$ & $44.07(5)$ \\
\hline $\mathrm{Cs} 2-\mathrm{O} 1-\mathrm{Cs} 3^{\mathrm{v}}$ & $99.05(8)$ \\
\hline $\mathrm{Cs} 1^{\mathrm{ix}}-\mathrm{O} 1-\mathrm{Cs} 3^{\mathrm{v}}$ & $108.26(8)$ \\
\hline $\mathrm{Cs} 2^{\mathrm{xiv}}-\mathrm{O} 1-\mathrm{Cs} 3^{\mathrm{v}}$ & $57.29(5)$ \\
\hline $\mathrm{Si} 3^{\mathrm{v}}-\mathrm{O} 1-\mathrm{Cs} 2^{\mathrm{v}}$ & $11.56(12)$ \\
\hline $\mathrm{Lu} 1-\mathrm{O} 1-\mathrm{Cs} 2^{\mathrm{v}}$ & $132.00(14)$ \\
\hline $\mathrm{Cs} 3-\mathrm{O} 1-\mathrm{Cs} 2^{\mathrm{v}}$ & $116.19(11)$ \\
\hline $\mathrm{Cs} 1^{\mathrm{xiv}}-\mathrm{O} 1-\mathrm{Cs} 2^{\mathrm{v}}$ & $85.77(8)$ \\
\hline $\mathrm{Cs} 2-\mathrm{O} 1-\mathrm{Cs} 2^{\mathrm{v}}$ & $104.28(8)$ \\
\hline $\mathrm{Cs} 1^{\mathrm{ix}}-\mathrm{O} 1-\mathrm{Cs} 2^{\mathrm{v}}$ & $70.55(6)$ \\
\hline $\mathrm{Cs} 2^{\mathrm{xiv}}-\mathrm{O} 1-\mathrm{Cs} 2^{\mathrm{v}}$ & $92.60(7)$ \\
\hline $\mathrm{Cs} 3^{\mathrm{v}}-\mathrm{O} 1-\mathrm{Cs} 2^{\mathrm{v}}$ & $43.78(3)$ \\
\hline $\mathrm{Si} 2^{\mathrm{vii}}-\mathrm{O} 2-\mathrm{Lu} 1$ & $139.2(2)$ \\
\hline $\mathrm{Si} 2^{\mathrm{vii}}-\mathrm{O} 2-\mathrm{Cs} 2^{\mathrm{xiv}}$ & $113.1(2)$ \\
\hline $\mathrm{Lu} 1-\mathrm{O} 2-\mathrm{Cs} 2^{2 \mathrm{xiv}}$ & $107.66(14)$ \\
\hline $\mathrm{Si} 2^{\mathrm{vii}}-\mathrm{O} 2-\mathrm{Cs} 1^{\mathrm{xiv}}$ & 91.77 (19) \\
\hline $\mathrm{Lu} 1-\mathrm{O} 2-\mathrm{Cs} 1^{\mathrm{xiv}}$ & $97.65(15)$ \\
\hline $\mathrm{Cs} 2^{\mathrm{xiv}}-\mathrm{O} 2-\mathrm{Cs} 1^{\mathrm{xiv}}$ & $77.51(10)$ \\
\hline $\mathrm{Si} 2^{\mathrm{vii}}-\mathrm{O} 2-\mathrm{Cs} 1^{\mathrm{xii}}$ & $92.29(19)$ \\
\hline $\mathrm{Lu} 1-\mathrm{O} 2-\mathrm{Cs} 1^{\mathrm{xii}}$ & $94.64(14)$ \\
\hline $\mathrm{Cs} 2^{\mathrm{xiv}}-\mathrm{O} 2-\mathrm{Cs} 1^{\mathrm{xii}}$ & $79.10(10)$ \\
\hline $\mathrm{Cs} 1^{\mathrm{xiv}}-\mathrm{O} 2-\mathrm{Cs} 1^{\mathrm{xii}}$ & $155.96(14)$ \\
\hline $\mathrm{Si} 2^{\mathrm{vii}}-\mathrm{O} 2-\mathrm{Cs} 3^{\mathrm{vii}}$ & $61.25(14)$ \\
\hline $\mathrm{Lu} 1-\mathrm{O} 2-\mathrm{Cs} 3^{\mathrm{vii}}$ & $93.74(12)$ \\
\hline $\mathrm{Cs} 2^{\mathrm{xiv}}-\mathrm{O} 2-\mathrm{Cs} 3^{\mathrm{vii}}$ & $126.68(13)$ \\
\hline $\mathrm{Cs} 1^{\mathrm{xiv}}-\mathrm{O} 2-\mathrm{Cs} 3^{\mathrm{vii}}$ & $148.21(12)$ \\
\hline $\mathrm{Cs} 1^{\mathrm{xii}}-\mathrm{O} 2-\mathrm{Cs} 3^{\mathrm{vii}}$ & $50.31(5)$ \\
\hline $\mathrm{Si} 2^{\mathrm{vii}}-\mathrm{O} 2-\mathrm{Cs} 3^{\mathrm{ix}}$ & $144.7(2)$ \\
\hline $\mathrm{Lu} 1-\mathrm{O} 2-\mathrm{Cs} 3^{\mathrm{ix}}$ & $64.56(10)$ \\
\hline $\mathrm{Cs} 2^{\mathrm{xiv}}-\mathrm{O} 2-\mathrm{Cs} 3^{\mathrm{ix}}$ & $53.55(7)$ \\
\hline $\mathrm{Cs} 1^{\mathrm{xiv}}-\mathrm{O} 2-\mathrm{Cs} 3^{\mathrm{ix}}$ & $113.05(10)$ \\
\hline $\mathrm{Cs} 1^{\mathrm{xii}}-\mathrm{O} 2-\mathrm{Cs} 3^{\mathrm{ix}}$ & $54.90(6)$ \\
\hline $\mathrm{Cs} 3^{\mathrm{vii}}-\mathrm{O} 2-\mathrm{Cs} 3^{\mathrm{ix}}$ & $98.65(9)$ \\
\hline $\mathrm{Si} 2^{\mathrm{vii}}-\mathrm{O} 2-\mathrm{Cs} 3^{\mathrm{viii}}$ & $44.36(14)$ \\
\hline $\mathrm{Lu} 1-\mathrm{O} 2-\mathrm{Cs} 3^{\mathrm{viii}}$ & $117.77(15)$ \\
\hline $\mathrm{Cs} 2^{\text {xiv }}-\mathrm{O} 2-\mathrm{Cs} 3^{\text {viii }}$ & $113.50(11)$ \\
\hline $\mathrm{Cs} 1^{\mathrm{xiv}}-\mathrm{O} 2-\mathrm{Cs} 3^{\mathrm{viii}}$ & $51.75(7)$ \\
\hline $\mathrm{Cs} 1^{\mathrm{xii}}-\mathrm{O} 2-\mathrm{Cs} 3^{\mathrm{viii}}$ & $136.66(10)$ \\
\hline $\mathrm{Cs} 3^{\mathrm{vii}}-\mathrm{O} 2-\mathrm{Cs} 3^{\text {viii }}$ & $96.80(7)$ \\
\hline $\mathrm{Cs} 3^{\mathrm{ix}}-\mathrm{O} 2-\mathrm{Cs} 3^{\text {viii }}$ & $164.19(9)$ \\
\hline $\mathrm{Si} 2^{\mathrm{vii}}-\mathrm{O} 2-\mathrm{Cs} 2^{\mathrm{vii}}$ & $15.48(12)$ \\
\hline $\mathrm{Lu} 1-\mathrm{O} 2-\mathrm{Cs} 2^{\mathrm{vii}}$ & $129.20(13)$ \\
\hline $\mathrm{Cs} 2^{\mathrm{xiv}}-\mathrm{O} 2-\mathrm{Cs} 2^{\mathrm{vii}}$ & $120.81(11)$ \\
\hline $\mathrm{Cs} 1^{\mathrm{xiv}}-\mathrm{O} 2-\mathrm{Cs} 2^{\mathrm{vii}}$ & $106.26(10)$ \\
\hline $\mathrm{Cs} 1^{\mathrm{xii}}-\mathrm{O} 2-\mathrm{Cs} 2^{\mathrm{vii}}$ & $81.19(8)$ \\
\hline $\mathrm{Cs} 3^{\mathrm{vii}}-\mathrm{O} 2-\mathrm{Cs} 2^{\mathrm{vii}}$ & $45.79(4)$ \\
\hline $\mathrm{Cs} 3^{\mathrm{ix}}-\mathrm{O} 2-\mathrm{Cs} 2^{\mathrm{vii}}$ & $135.98(10)$ \\
\hline
\end{tabular}




\begin{tabular}{|c|c|c|c|}
\hline $\mathrm{Cs} 3-\mathrm{Cs} 2-\mathrm{O} 4^{\mathrm{i}}$ & $78.05(6)$ & $\mathrm{Cs} 3^{\mathrm{viii}}-\mathrm{O} 2-\mathrm{Cs} 2^{\mathrm{vii}}$ & $56.35(4)$ \\
\hline $\mathrm{Si} 2^{\mathrm{v}}-\mathrm{Cs} 2-\mathrm{O} 4^{\mathrm{i}}$ & $22.85(6)$ & $\mathrm{Si} 2^{\mathrm{vii}}-\mathrm{O} 2-\mathrm{Cs} 3$ & $109.84(18)$ \\
\hline 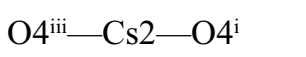 & $114.71(8)$ & $\mathrm{Lu} 1-\mathrm{O} 2-\mathrm{Cs} 3$ & $35.50(8)$ \\
\hline $\mathrm{O} 2^{\mathrm{ii}}-\mathrm{Cs} 2-\mathrm{Cs} 1^{\mathrm{xiv}}$ & $56.67(9)$ & $\mathrm{Cs} 2^{\mathrm{xiv}}-\mathrm{O} 2-\mathrm{Cs} 3$ & $131.18(11)$ \\
\hline $\mathrm{O} 9^{\mathrm{iii}}-\mathrm{Cs} 2-\mathrm{Cs} 1^{\mathrm{xiv}}$ & $79.73(7)$ & $\mathrm{Cs} 11^{\mathrm{xiv}}-\mathrm{O} 2-\mathrm{Cs} 3$ & $79.05(9)$ \\
\hline $\mathrm{O} 6-\mathrm{Cs} 2-\mathrm{Cs} 1^{\mathrm{xiv}}$ & $145.22(8)$ & $\mathrm{Cs} 1{ }^{\mathrm{xii}}-\mathrm{O} 2-\mathrm{Cs} 3$ & $121.43(10)$ \\
\hline $\mathrm{O} 8-\mathrm{Cs} 2-\mathrm{Cs} 1^{\mathrm{xiv}}$ & $127.74(7)$ & $\mathrm{Cs} 33^{\mathrm{vii}}-\mathrm{O} 2-\mathrm{Cs} 3$ & $93.90(7)$ \\
\hline $\mathrm{O} 5^{\mathrm{iii}}-\mathrm{Cs} 2-\mathrm{Cs} 1^{\mathrm{xiv}}$ & $128.48(8)$ & $\mathrm{Cs} 3^{\mathrm{ix}}-\mathrm{O} 2-\mathrm{Cs} 3$ & $99.66(7)$ \\
\hline $\mathrm{O} 7-\mathrm{Cs} 2-\mathrm{Cs} 1^{\mathrm{xiv}}$ & $85.99(7)$ &  & $82.57(7)$ \\
\hline $\mathrm{Si} 3-\mathrm{Cs} 2-\mathrm{Cs} 1^{\mathrm{xiv}}$ & $113.08(3)$ & $\mathrm{Cs} 2{ }^{\mathrm{vii}}-\mathrm{O} 2-\mathrm{Cs} 3$ & $106.64(7)$ \\
\hline $\mathrm{Si} 2-\mathrm{Cs} 2-\mathrm{Cs} 1^{\mathrm{xiv}}$ & $152.34(3)$ & $\mathrm{Si} 2^{\mathrm{vii}}-\mathrm{O} 2-\mathrm{Cs} 2^{\mathrm{x}}$ & $94.90(16)$ \\
\hline $\mathrm{Si} 1^{\mathrm{iii}}-\mathrm{Cs} 2-\mathrm{Cs} 1^{\mathrm{xiv}}$ & $104.07(3)$ & $\mathrm{Lu} 1-\mathrm{O} 2-\mathrm{Cs} 2^{\mathrm{x}}$ & $50.45(8)$ \\
\hline $\mathrm{O} 3^{\mathrm{vii}}-\mathrm{Cs} 2-\mathrm{Cs} 1^{\mathrm{xiv}}$ & $120.44(7)$ & $\mathrm{Cs} 2^{\mathrm{xiv}}-\mathrm{O} 2-\mathrm{Cs} 2^{\mathrm{x}}$ & $139.86(13)$ \\
\hline $\mathrm{Cs} 1-\mathrm{Cs} 2-\mathrm{Cs} 1^{\mathrm{xiv}}$ & $108.832(10)$ & $\mathrm{Cs} 1^{\mathrm{xiv}}-\mathrm{O} 2-\mathrm{Cs} 2^{\mathrm{x}}$ & $131.88(12)$ \\
\hline $\mathrm{O} 1-\mathrm{Cs} 2-\mathrm{Cs} 1^{\mathrm{xiv}}$ & $44.06(6)$ & $\mathrm{Cs} 1^{\mathrm{xii}}-\mathrm{O} 2-\mathrm{Cs} 2^{\mathrm{x}}$ & $71.28(7)$ \\
\hline 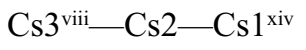 & $59.406(10)$ & $\mathrm{Cs} 3^{\mathrm{vii}}-\mathrm{O} 2-\mathrm{Cs} 2^{\mathrm{x}}$ & $44.37(4)$ \\
\hline $\mathrm{Cs} 3-\mathrm{Cs} 2-\mathrm{Cs} 1^{\mathrm{xiv}}$ & $88.161(13)$ & $\mathrm{Cs} 3^{\mathrm{ix}}-\mathrm{O} 2-\mathrm{Cs} 2^{\mathrm{x}}$ & $86.90(7)$ \\
\hline $\mathrm{Si} 2^{\mathrm{v}}-\mathrm{Cs} 2-\mathrm{Cs} 1^{\mathrm{xiv}}$ & $58.63(2)$ & 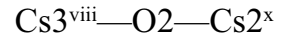 & $106.64(7)$ \\
\hline $\mathrm{O} 4^{\mathrm{iii}}-\mathrm{Cs} 2-\mathrm{Cs} 1^{\mathrm{xiv}}$ & $165.72(6)$ & $\mathrm{Cs} 2^{\mathrm{vii}}-\mathrm{O} 2-\mathrm{Cs} 2^{\mathrm{x}}$ & $81.15(5)$ \\
\hline $\mathrm{O} 4^{\mathrm{i}}-\mathrm{Cs} 2-\mathrm{Cs}^{1 \mathrm{xiv}}$ & $79.01(6)$ & $\mathrm{Cs} 3-\mathrm{O} 2-\mathrm{Cs} 2^{\mathrm{x}}$ & $53.91(4)$ \\
\hline $\mathrm{O} 2^{\mathrm{ii}}-\mathrm{Cs} 2-\mathrm{Lu} 1^{\mathrm{ii}}$ & $29.98(8)$ & $\mathrm{Si}^{\mathrm{iv}}-\mathrm{O} 3-\mathrm{Lu}^{\mathrm{vi}}$ & $139.2(3)$ \\
\hline $\mathrm{O} 9^{\mathrm{iii}}-\mathrm{Cs} 2-\mathrm{Lu} 1^{\mathrm{ii}}$ & $30.64(8)$ & $\mathrm{Si}^{3 \mathrm{iv}}-\mathrm{O} 3-\mathrm{Cs} 1$ & $103.2(2)$ \\
\hline $\mathrm{O} 6-\mathrm{Cs} 2-\mathrm{Lu} 1^{\mathrm{ii}}$ & $131.47(9)$ & 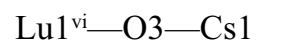 & $117.44(16)$ \\
\hline $\mathrm{O} 8-\mathrm{Cs} 2-\mathrm{Lu} 1^{\mathrm{ii}}$ & $165.07(7)$ & $\mathrm{Si}^{\mathrm{iv}}-\mathrm{O} 3-\mathrm{Cs} 3^{\mathrm{i}}$ & 89.78 (19) \\
\hline $\mathrm{O} 5^{\mathrm{iii}}-\mathrm{Cs} 2-\mathrm{Lu} 1^{\mathrm{ii}}$ & $76.86(7)$ & $\mathrm{Lu} 1^{\mathrm{vi}}-\mathrm{O} 3-\mathrm{Cs} 3^{\mathrm{i}}$ & 100.59 \\
\hline $\mathrm{O} 7-\mathrm{Cs} 2-\mathrm{Lu} 1^{\mathrm{ii}}$ & $130.90(7)$ & $\mathrm{Cs} 1-\mathrm{O} 3-\mathrm{Cs} 3^{\mathrm{i}}$ & $79.85(11)$ \\
\hline $\mathrm{Si} 3-\mathrm{Cs} 2-\mathrm{Lu} 1^{\mathrm{ii}}$ & $143.66(3)$ & $\mathrm{Si} 3^{\mathrm{iv}}-\mathrm{O} 3-\mathrm{Cs} 2^{\mathrm{iv}}$ & $73.28(16)$ \\
\hline $\mathrm{Si} 2-\mathrm{Cs} 2-\mathrm{Lu} 1^{\mathrm{ii}}$ & $145.25(3)$ & $\mathrm{Lu} 1^{\mathrm{vi}}-\mathrm{O} 3-\mathrm{Cs} 2^{\mathrm{iv}}$ & $89.12(14)$ \\
\hline 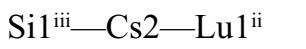 & $53.07(2)$ & $\mathrm{Cs} 1-\mathrm{O} 3-\mathrm{Cs} 2^{\mathrm{iv}}$ & $109.09(13)$ \\
\hline $\mathrm{O}^{\mathrm{vii}}-\mathrm{Cs} 2-\mathrm{Lu} 1^{\mathrm{ii}}$ & $122.46(7)$ & $\mathrm{Cs} 33^{\mathrm{i}}-\mathrm{O} 3-\mathrm{Cs} 2^{\mathrm{iv}}$ & $162.11(13)$ \\
\hline $\mathrm{Cs} 1-\mathrm{Cs} 2-\mathrm{Lu} 1^{\mathrm{ii}}$ & $59.975(10)$ & $\mathrm{Si} 3^{\mathrm{iv}}-\mathrm{O} 3-\mathrm{Cs} 3^{\mathrm{iii}}$ & $98.58(18)$ \\
\hline $\mathrm{O} 1-\mathrm{Cs} 2-\mathrm{Lu} 1^{\mathrm{ii}}$ & $97.88(6)$ & $\mathrm{Lu} 1^{\mathrm{vi}}-\mathrm{O} 3-\mathrm{Cs} 3^{\mathrm{iii}}$ & $101.10(14)$ \\
\hline $\mathrm{Cs} 3^{\text {viii }}-\mathrm{Cs} 2-\mathrm{Lu} 1^{\mathrm{ii}}$ & $64.629(10)$ & $\mathrm{Cs} 1-\mathrm{O} 3-\mathrm{Cs} 3^{\mathrm{iii}}$ & $55.28(8)$ \\
\hline $\mathrm{Cs} 3-\mathrm{Cs} 2-\mathrm{Lu} 1^{\mathrm{ii}}$ & $138.708(14)$ & $\mathrm{Cs} 3^{\mathrm{i}}-\mathrm{O} 3-\mathrm{Cs} 3^{\mathrm{iii}}$ & $135.11(12)$ \\
\hline $\mathrm{Si} 2^{\mathrm{v}}-\mathrm{Cs} 2-\mathrm{Lu} 1^{\mathrm{ii}}$ & $52.22(2)$ & $\mathrm{Cs} 2^{\mathrm{iv}}-\mathrm{O} 3-\mathrm{Cs} 3^{\mathrm{iii}}$ & $55.62(6)$ \\
\hline $\mathrm{O} 4^{\mathrm{iii}}-\mathrm{Cs} 2-\mathrm{Lu} 1^{\mathrm{ii}}$ & $124.89(6)$ & $\mathrm{Si} 3^{\mathrm{iv}}-\mathrm{O} 3-\mathrm{Cs} 2^{\mathrm{ii}}$ & $143.6(2)$ \\
\hline $\mathrm{O} 4{ }^{\mathrm{i}}-\mathrm{Cs} 2-\mathrm{Lu} 1^{\mathrm{ii}}$ & $68.93(6)$ & $\mathrm{Lu} 1^{\mathrm{vi}}-\mathrm{O} 3-\mathrm{Cs} 2^{2 i}$ & $66.29(10)$ \\
\hline $\mathrm{Cs} 1^{\mathrm{xiv}}-\mathrm{Cs} 2-\mathrm{Lu} 1^{\mathrm{ii}}$ & $62.263(9)$ & $\mathrm{Cs} 1-\mathrm{O} 3-\mathrm{Cs} 2^{\mathrm{ii}}$ & $63.70(8)$ \\
\hline $\mathrm{O} 2^{\mathrm{ii}}-\mathrm{Cs} 2-\mathrm{O}^{\mathrm{v}}$ & $38.65(10)$ & $\mathrm{Cs} 3-\mathrm{O} 3-\mathrm{Cs} 2^{\mathrm{ii}}$ & $55.48(6)$ \\
\hline 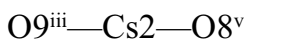 & $95.23(9)$ & $\mathrm{Cs} 2^{\mathrm{iv}}-\mathrm{O} 3-\mathrm{Cs} 2^{\mathrm{ii}}$ & $142.24(12)$ \\
\hline $\mathrm{O} 6-\mathrm{Cs} 2-\mathrm{O}^{\mathrm{v}}$ & $108.13(9)$ & $\mathrm{Cs} 3^{\mathrm{iii}}-\mathrm{O} 3-\mathrm{Cs} 2^{\mathrm{ii}}$ & $99.72(8)$ \\
\hline $\mathrm{O} 8-\mathrm{Cs} 2-\mathrm{O}^{\mathrm{v}}$ & $129.66(6)$ & $\mathrm{Si} 3^{\mathrm{iv}}-\mathrm{O} 3-\mathrm{Cs} 2^{\mathrm{i}}$ & $68.55(16)$ \\
\hline 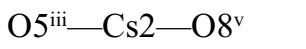 & $139.20(9)$ & $\mathrm{Lu} 1^{\mathrm{vi}}-\mathrm{O} 3-\mathrm{Cs} 2^{\mathrm{i}}$ & $86.51(12)$ \\
\hline $\mathrm{O} 7-\mathrm{Cs} 2-\mathrm{O}^{\mathrm{v}}$ & $115.96(9)$ & $\mathrm{Cs} 1-\mathrm{O} 3-\mathrm{Cs} 2^{\mathrm{i}}$ & $131.12(14)$ \\
\hline $\mathrm{Si} 3-\mathrm{Cs} 2-\mathrm{O}^{\mathrm{v}}$ & $138.41(6)$ & $\mathrm{Cs} 3^{\mathrm{i}}-\mathrm{O} 3-\mathrm{Cs} 2^{\mathrm{i}}$ & $52.98(6)$ \\
\hline $\mathrm{Si} 2-\mathrm{Cs} 2-\mathrm{O}^{\mathrm{v}}$ & $131.61(6)$ & $\mathrm{Cs} 2^{\mathrm{iv}}-\mathrm{O} 3-\mathrm{Cs} 2^{\mathrm{i}}$ & $113.46(10)$ \\
\hline $\mathrm{Si}^{\mathrm{iii}}-\mathrm{Cs} 2-\mathrm{O}^{\mathrm{v}}$ & $118.10(6)$ & $\mathrm{Cs} 3^{\mathrm{iii}}-\mathrm{O} 3-\mathrm{Cs} 2^{\mathrm{i}}$ & $166.07(10)$ \\
\hline $\mathrm{O} 3^{\mathrm{vii}}-\mathrm{Cs} 2-\mathrm{O}^{\mathrm{v}}$ & $159.22(8)$ & $\mathrm{Cs} 2^{\mathrm{ii}}-\mathrm{O} 3-\mathrm{Cs} 2^{\mathrm{i}}$ & $94.02(8)$ \\
\hline
\end{tabular}




\begin{tabular}{|c|c|}
\hline $\mathrm{Cs} 1-\mathrm{Cs} 2-\mathrm{O} 8^{\mathrm{v}}$ & $76.81(5)$ \\
\hline $\mathrm{O} 1-\mathrm{Cs} 2-\mathrm{O} 8^{\mathrm{v}}$ & $38.53(8)$ \\
\hline $\mathrm{Cs} 3^{\mathrm{viii}}-\mathrm{Cs} 2-\mathrm{O}^{\mathrm{v}}$ & $99.68(5)$ \\
\hline $\mathrm{Cs} 3-\mathrm{Cs} 2-\mathrm{O} 8^{\mathrm{v}}$ & $73.48(5)$ \\
\hline $\mathrm{Si} 2^{\mathrm{v}}-\mathrm{Cs} 2-\mathrm{O}^{\mathrm{v}}$ & $23.12(6)$ \\
\hline 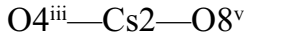 & $149.51(8)$ \\
\hline $\mathrm{O} 4^{\mathrm{i}}-\mathrm{Cs} 2-\mathrm{O}^{\mathrm{v}}$ & $37.39(8)$ \\
\hline $\mathrm{Cs} 1^{\mathrm{xiv}}-\mathrm{Cs} 2-\mathrm{O} 8^{\mathrm{v}}$ & $42.75(5)$ \\
\hline $\mathrm{Lu} 1^{\mathrm{ii}}-\mathrm{Cs} 2-\mathrm{O}^{\mathrm{v}}$ & $65.24(5)$ \\
\hline $\mathrm{O} 2^{\mathrm{ii}}-\mathrm{Cs} 2-\mathrm{O}^{\mathrm{iv}}$ & $89.10(10)$ \\
\hline $\mathrm{O} 9^{\mathrm{iii}}-\mathrm{Cs} 2-\mathrm{O} 9^{\mathrm{iv}}$ & $138.56(12)$ \\
\hline $\mathrm{O} 6-\mathrm{Cs} 2-\mathrm{O}^{\text {iv }}$ & $38.75(9)$ \\
\hline $\mathrm{O} 8-\mathrm{Cs} 2-\mathrm{O}^{\mathrm{iv}}$ & $73.20(9)$ \\
\hline $\mathrm{O} 5^{\mathrm{iii}}-\mathrm{Cs} 2-\mathrm{O}^{\mathrm{iv}}$ & $118.34(10)$ \\
\hline $\mathrm{O} 7-\mathrm{Cs} 2-\mathrm{O}^{\mathrm{iv}}$ & $108.15(9)$ \\
\hline $\mathrm{Si} 3-\mathrm{Cs} 2-\mathrm{O}^{\mathrm{iv}}$ & $99.56(6)$ \\
\hline $\mathrm{Si} 2-\mathrm{Cs} 2-\mathrm{O}^{\mathrm{iv}}$ & $62.44(6)$ \\
\hline $\mathrm{Si} 1^{\mathrm{iii}}-\mathrm{Cs} 2-\mathrm{O}^{\mathrm{iv}}$ & $132.99(6)$ \\
\hline $\mathrm{O} 3^{\mathrm{vii}}-\mathrm{Cs} 2-\mathrm{O}^{\mathrm{iv}}$ & $115.71(9)$ \\
\hline $\mathrm{Cs} 1-\mathrm{Cs} 2-\mathrm{O}^{\mathrm{iv}}$ & $67.93(6)$ \\
\hline $\mathrm{O} 1-\mathrm{Cs} 2-\mathrm{O}^{\mathrm{iv}}$ & $68.78(8)$ \\
\hline $\mathrm{Cs} 3^{\text {viii }}-\mathrm{Cs} 2-\mathrm{O} 9^{\mathrm{iv}}$ & $165.78(6)$ \\
\hline $\mathrm{Cs} 3-\mathrm{Cs} 2-\mathrm{O}^{\mathrm{iv}}$ & $43.01(5)$ \\
\hline $\mathrm{Si} 2^{\mathrm{v}}-\mathrm{Cs} 2-\mathrm{O}^{\mathrm{iv}}$ & $68.90(6)$ \\
\hline $\mathrm{O} 4^{\mathrm{iii}}-\mathrm{Cs} 2-\mathrm{O}^{\mathrm{iv}}$ & $80.87(8)$ \\
\hline $\mathrm{O} 4-\mathrm{Cs} 2-\mathrm{O}^{\mathrm{iv}}$ & $48.22(8)$ \\
\hline $\mathrm{Cs} 1^{\mathrm{xiv}}-\mathrm{Cs} 2-\mathrm{O} 9^{\mathrm{iv}}$ & $107.67(6)$ \\
\hline $\mathrm{Lu}{ }^{1 \mathrm{ii}}-\mathrm{Cs} 2-\mathrm{O}^{\mathrm{iv}}$ & $116.42(6)$ \\
\hline $\mathrm{O} 8^{\mathrm{v}}-\mathrm{Cs} 2-\mathrm{O}^{\mathrm{iv}}$ & $69.78(8)$ \\
\hline $\mathrm{O} 2^{\mathrm{ii}}-\mathrm{Cs} 2-\mathrm{Si}^{\mathrm{iv}}$ & $101.18(9)$ \\
\hline $\mathrm{O} 9^{\mathrm{iii}}-\mathrm{Cs} 2-\mathrm{Si}^{\mathrm{iv}}$ & $132.27(8)$ \\
\hline $\mathrm{O} 6-\mathrm{Cs} 2-\mathrm{Si}^{\mathrm{iv}}$ & $18.14(7)$ \\
\hline $\mathrm{O} 8-\mathrm{Cs} 2-\mathrm{Si}^{\mathrm{iv}}$ & $62.24(7)$ \\
\hline $\mathrm{O} 5^{\mathrm{iii}}-\mathrm{Cs} 2-\mathrm{Si}^{\mathrm{iv}}$ & $98.14(8)$ \\
\hline $\mathrm{O} 7-\mathrm{Cs} 2-\mathrm{Si}^{\mathrm{iv}}$ & $106.69(7)$ \\
\hline $\mathrm{Si} 3-\mathrm{Cs} 2-\mathrm{Si}^{\mathrm{iv}}$ & $89.40(3)$ \\
\hline $\mathrm{Si} 2-\mathrm{Cs} 2-\mathrm{Si}^{\mathrm{iv}}$ & $44.31(3)$ \\
\hline $\mathrm{Si} 1^{\mathrm{iii}}-\mathrm{Cs} 2-\mathrm{Si}^{\mathrm{iv}}$ & $117.07(4)$ \\
\hline $\mathrm{O}^{\mathrm{vii}}-\mathrm{Cs} 2-\mathrm{Si}^{\mathrm{iv}}$ & $99.23(7)$ \\
\hline $\mathrm{Cs} 1-\mathrm{Cs} 2-\mathrm{Si}^{\mathrm{iv}}$ & $64.09(2)$ \\
\hline $\mathrm{O} 1-\mathrm{Cs} 2-\mathrm{Si}^{\mathrm{iv}}$ & $89.26(7)$ \\
\hline $\mathrm{Cs} 3^{\mathrm{viii}}-\mathrm{Cs} 2-\mathrm{Si} 1^{\mathrm{iv}}$ & $169.99(2)$ \\
\hline $\mathrm{Cs} 3-\mathrm{Cs} 2-\mathrm{Si}^{\mathrm{iv}}$ & $54.81(2)$ \\
\hline $\mathrm{Si} 2^{\mathrm{v}}-\mathrm{Cs} 2-\mathrm{Si}^{\mathrm{iv}}$ & $84.51(3)$ \\
\hline $\mathrm{O} 4^{\mathrm{iii}}-\mathrm{Cs} 2-\mathrm{Si}^{\mathrm{iv}}$ & $59.82(6)$ \\
\hline $\mathrm{O} 4-\mathrm{Cs} 2-\mathrm{Si}^{\mathrm{i}}{ }^{\mathrm{iv}}$ & $61.86(6)$ \\
\hline $\mathrm{Cs} 1^{\mathrm{xiv}}-\mathrm{Cs} 2-\mathrm{Si} 1^{\mathrm{iv}}$ & $129.33(2)$ \\
\hline $\mathrm{Lu} 1^{\mathrm{ii}}-\mathrm{Cs} 2-\mathrm{Si}^{\mathrm{iv}}$ & $122.36(2)$ \\
\hline
\end{tabular}

\begin{tabular}{|c|c|}
\hline $\mathrm{Si}^{3 \mathrm{iv}}-\mathrm{O} 3-\mathrm{Cs}^{\mathrm{xi}}$ & $18.67(12)$ \\
\hline $\mathrm{Lu} 1^{\mathrm{vi}}-\mathrm{O} 3-\mathrm{Cs} 1^{\mathrm{xi}}$ & $124.64(15)$ \\
\hline $\mathrm{Cs} 1-\mathrm{O} 3-\mathrm{Cs} 1^{\mathrm{xi}}$ & $117.25(11)$ \\
\hline $\mathrm{Cs} 3-\mathrm{O} 3-\mathrm{Cs}^{\mathrm{xi}}$ & $80.99(8)$ \\
\hline $\mathrm{Cs} 2^{\mathrm{iv}}-\mathrm{O} 3-\mathrm{Cs} 1^{\mathrm{xi}}$ & $81.13(7)$ \\
\hline $\mathrm{Cs} 3^{\mathrm{iii}}-\mathrm{O} 3-\mathrm{Cs}^{\mathrm{xi}}$ & $116.34(9)$ \\
\hline $\mathrm{Cs} 2^{\mathrm{ii}}-\mathrm{O} 3-\mathrm{Cs} 1^{\mathrm{xi}}$ & $136.22(10)$ \\
\hline $\mathrm{Cs} 2^{\mathrm{i}}-\mathrm{O} 3-\mathrm{Cs}^{\mathrm{xi}}$ & $50.34(4)$ \\
\hline $\mathrm{Si}^{\mathrm{iv}}-\mathrm{O} 3-\mathrm{Cs} 3^{\mathrm{vi}}$ & $110.12(19)$ \\
\hline $\mathrm{Lu} 1^{\mathrm{vi}}-\mathrm{O} 3-\mathrm{Cs} 3^{\mathrm{vi}}$ & $32.72(8)$ \\
\hline $\mathrm{Cs} 1-\mathrm{O} 3-\mathrm{Cs} 3^{\mathrm{vi}}$ & $138.99(13)$ \\
\hline $\mathrm{Cs} 3-\mathrm{O} 3-\mathrm{Cs} 3^{\mathrm{vi}}$ & $122.52(11)$ \\
\hline $\mathrm{Cs} 2^{\mathrm{iv}}-\mathrm{O} 3-\mathrm{Cs} 3^{\mathrm{vi}}$ & $60.87(6)$ \\
\hline $\mathrm{Cs} 3^{\mathrm{iii}}-\mathrm{O} 3-\mathrm{Cs} 3^{\mathrm{vi}}$ & $95.80(8)$ \\
\hline $\mathrm{Cs} 2^{\mathrm{ii}}-\mathrm{O} 3-\mathrm{Cs} 3^{\mathrm{vi}}$ & $99.02(7)$ \\
\hline $\mathrm{Cs} 2^{\mathrm{i}}-\mathrm{O} 3-\mathrm{Cs} 3^{\mathrm{vi}}$ & $84.26(7)$ \\
\hline $\mathrm{Cs} 1^{\mathrm{xi}}-\mathrm{O} 3-\mathrm{Cs} 3^{\mathrm{vi}}$ & $101.02(7)$ \\
\hline $\mathrm{Si} 2^{\mathrm{x}}-\mathrm{O} 4-\mathrm{Lu} 1$ & $138.2(2)$ \\
\hline $\mathrm{Si} 2^{\mathrm{x}}-\mathrm{O} 4-\mathrm{Cs} 1^{\mathrm{ix}}$ & $95.68(18)$ \\
\hline $\mathrm{Lu} 1-\mathrm{O} 4-\mathrm{Cs} 1^{\mathrm{ix}}$ & $119.96(17)$ \\
\hline $\mathrm{Si} 2^{\mathrm{x}}-\mathrm{O} 4-\mathrm{Cs} 3$ & $119.6(2)$ \\
\hline $\mathrm{Lu} 1-\mathrm{O} 4-\mathrm{Cs} 3$ & $88.09(13)$ \\
\hline $\mathrm{Cs} 11^{\mathrm{ix}}-\mathrm{O} 4-\mathrm{Cs} 3$ & $82.89(10)$ \\
\hline $\mathrm{Si} 2^{\mathrm{x}}-\mathrm{O} 4-\mathrm{Cs} 2^{\mathrm{x}}$ & $68.15(15)$ \\
\hline Lu1-O4-Cs2 ${ }^{\mathrm{x}}$ & $84.32(12)$ \\
\hline $\mathrm{Cs} 1^{\mathrm{ix}}-\mathrm{O} 4-\mathrm{Cs} 2^{\mathrm{x}}$ & $153.06(14)$ \\
\hline $\mathrm{Cs} 3-\mathrm{O} 4-\mathrm{Cs} 2^{\mathrm{x}}$ & $86.77(10)$ \\
\hline $\mathrm{Si} 2^{\mathrm{x}}-\mathrm{O} 4-\mathrm{Cs} 2^{\mathrm{ix}}$ & $71.29(16)$ \\
\hline $\mathrm{Lu} 1-\mathrm{O} 4-\mathrm{Cs} 2^{\mathrm{ix}}$ & $104.76(15)$ \\
\hline $\mathrm{Cs} 1^{\mathrm{ix}}-\mathrm{O} 4-\mathrm{Cs} 2^{\mathrm{ix}}$ & $62.36(8)$ \\
\hline $\mathrm{Cs} 3-\mathrm{O} 4-\mathrm{Cs} 2^{\mathrm{ix}}$ & $144.96(13)$ \\
\hline $\mathrm{Cs} 2^{\mathrm{x}}-\mathrm{O} 4-\mathrm{Cs} 2^{\mathrm{ix}}$ & $126.30(10)$ \\
\hline $\mathrm{Si} 2^{\mathrm{x}}-\mathrm{O} 4-\mathrm{Cs} 3^{\mathrm{ix}}$ & $95.57(17)$ \\
\hline $\mathrm{Lu} 1-\mathrm{O} 4-\mathrm{Cs} 3^{\mathrm{ix}}$ & $59.22(10)$ \\
\hline $\mathrm{Cs} 1^{\mathrm{ix}}-\mathrm{O} 4-\mathrm{Cs} 3^{\mathrm{ix}}$ & $100.48(11)$ \\
\hline $\mathrm{Cs} 3-\mathrm{O} 4-\mathrm{Cs} 3^{\mathrm{ix}}$ & $144.32(12)$ \\
\hline $\mathrm{Cs} 2^{\mathrm{x}}-\mathrm{O} 4-\mathrm{Cs} 3^{\mathrm{ix}}$ & $102.39(8)$ \\
\hline $\mathrm{Cs} 2^{\mathrm{ix}}-\mathrm{O} 4-\mathrm{Cs} 3^{\mathrm{ix}}$ & $48.73(5)$ \\
\hline $\mathrm{Si} 2^{\mathrm{x}}-\mathrm{O} 4-\mathrm{Cs} 1^{\mathrm{x}}$ & $86.04(18)$ \\
\hline $\mathrm{Lu} 1-\mathrm{O} 4-\mathrm{Cs} 1^{\mathrm{x}}$ & $97.52(13)$ \\
\hline $\mathrm{Cs} 1^{\mathrm{ix}}-\mathrm{O} 4-\mathrm{Cs} 1^{\mathrm{x}}$ & $113.68(11)$ \\
\hline $\mathrm{Cs} 3-\mathrm{O} 4-\mathrm{Cs} 1^{\mathrm{x}}$ & $43.21(6)$ \\
\hline $\mathrm{Cs} 2^{\mathrm{x}}-\mathrm{O} 4-\mathrm{Cs} 1^{\mathrm{x}}$ & $46.48(5)$ \\
\hline $\mathrm{Cs} 2^{\mathrm{ix}}-\mathrm{O} 4-\mathrm{Cs} 1^{\mathrm{x}}$ & $155.91(10)$ \\
\hline $\mathrm{Cs} 3^{\mathrm{ix}}-\mathrm{O} 4-\mathrm{Cs} 1^{\mathrm{x}}$ & $145.50(9)$ \\
\hline $\mathrm{Si} 2^{\mathrm{x}}-\mathrm{O} 4-\mathrm{Cs} 1^{\mathrm{xv}}$ & $29.68(13)$ \\
\hline $\mathrm{Lu} 1-\mathrm{O} 4-\mathrm{Cs} 1^{\mathrm{xv}}$ & $115.56(13)$ \\
\hline $\mathrm{Cs} 1^{1 \mathrm{x}}-\mathrm{O} 4-\mathrm{Cs}^{\mathrm{xv}}$ & $100.12(10)$ \\
\hline
\end{tabular}




\begin{tabular}{|c|c|}
\hline $\mathrm{O} 8^{\mathrm{v}}-\mathrm{Cs} 2-\mathrm{Si}^{\mathrm{iv}}$ & $90.09(6)$ \\
\hline $\mathrm{O} 9^{\mathrm{iv}}-\mathrm{Cs} 2-\mathrm{Si}^{\mathrm{iv}}$ & $21.67(6)$ \\
\hline $\mathrm{O} 2^{\mathrm{ii}}-\mathrm{Cs} 2-\mathrm{Lu} 1^{\mathrm{iii}}$ & $130.86(8)$ \\
\hline 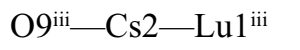 & $76.58(8)$ \\
\hline $\mathrm{O} 6-\mathrm{Cs} 2-\mathrm{Lu} 1^{\mathrm{iii}}$ & $73.00(7)$ \\
\hline $\mathrm{O} 8-\mathrm{Cs} 2-\mathrm{Lu} 1^{\mathrm{iii}}$ & $59.44(7)$ \\
\hline 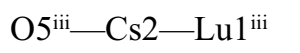 & $30.21(7)$ \\
\hline $\mathrm{O} 7-\mathrm{Cs} 2-\mathrm{Lu} 1^{\mathrm{iii}}$ & $74.19(7)$ \\
\hline $\mathrm{Si} 3-\mathrm{Cs} 2-\mathrm{Lu} 1^{\mathrm{iii}}$ & $52.40(2)$ \\
\hline $\mathrm{Si} 2-\mathrm{Cs} 2-\mathrm{Lu} 1^{\mathrm{iii}}$ & $52.72(2)$ \\
\hline $\mathrm{Si} 1^{\mathrm{iii}}-\mathrm{Cs} 2-\mathrm{Lu} 1^{\mathrm{iii}}$ & $52.58(2)$ \\
\hline $\mathrm{O} 3^{\mathrm{vii}}-\mathrm{Cs} 2-\mathrm{Lu} 1^{\mathrm{iii}}$ & $30.64(6)$ \\
\hline $\mathrm{Cs} 1-\mathrm{Cs} 2-\mathrm{Lu} 1^{\mathrm{iii}}$ & $93.689(13)$ \\
\hline $\mathrm{O} 1-\mathrm{Cs} 2-\mathrm{Lu} 1^{\mathrm{iii}}$ & $152.28(6)$ \\
\hline $\mathrm{Cs} 3^{\mathrm{viii}}-\mathrm{Cs} 2-\mathrm{Lu} 1^{\mathrm{iii}}$ & $80.477(11)$ \\
\hline Cs $3-\mathrm{Cs} 2-\mathrm{Lu} 1^{\mathrm{iii}}$ & $115.287(12)$ \\
\hline $\mathrm{Si} 2^{\mathrm{v}}-\mathrm{Cs} 2-\mathrm{Lu} 1^{i i i}$ & $146.38(3)$ \\
\hline 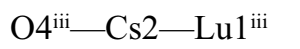 & $30.99(6)$ \\
\hline $\mathrm{O} 4{ }^{\mathrm{i}}-\mathrm{Cs} 2-\mathrm{Lu} 1^{\mathrm{iii}}$ & $135.44(6)$ \\
\hline $\mathrm{Cs} 1^{\mathrm{xiv}}-\mathrm{Cs} 2-\mathrm{Lu} 1^{\mathrm{iii}}$ & $139.705(14)$ \\
\hline $\mathrm{Lu} 1^{\mathrm{ii}}-\mathrm{Cs} 2-\mathrm{Lu} 1^{\mathrm{iii}}$ & 105.654 \\
\hline $\mathrm{O} 8^{v}-\mathrm{Cs} 2-\mathrm{Lu} 1^{\mathrm{iii}}$ & $169.19(5)$ \\
\hline $\mathrm{O} 9^{\mathrm{iv}}-\mathrm{Cs} 2-\mathrm{Lu} 1^{\mathrm{iii}}$ & $111.71(6)$ \\
\hline $\mathrm{Si} 1^{\mathrm{iv}}-\mathrm{Cs} 2-\mathrm{Lu} 1^{\mathrm{iii}}$ & $90.34(2)$ \\
\hline $\mathrm{O} 22^{\mathrm{ii}}-\mathrm{Cs} 2-\mathrm{Si} 1$ & $127.90(9)$ \\
\hline $\mathrm{O} 9^{\mathrm{iii}}-\mathrm{Cs} 2-\mathrm{Si} 1$ & $118.19(8)$ \\
\hline $\mathrm{O} 6-\mathrm{Cs} 2-\mathrm{Si} 1$ & $102.32(9)$ \\
\hline $\mathrm{O} 8-\mathrm{Cs} 2-\mathrm{Si} 1$ & $58.07(7)$ \\
\hline $\mathrm{O}^{\mathrm{iii}}-\mathrm{Cs} 2-\mathrm{Si} 1$ & $116.48(8)$ \\
\hline $\mathrm{O} 7-\mathrm{Cs} 2-\mathrm{Si} 1$ & $17.58(8)$ \\
\hline $\mathrm{Si} 3-\mathrm{Cs} 2-\mathrm{Si} 1$ & $43.03(3)$ \\
\hline $\mathrm{Si} 2-\mathrm{Cs} 2-\mathrm{Si} 1$ & $85.06(3)$ \\
\hline $\mathrm{Si} 1^{\mathrm{iii}}-\mathrm{Cs} 2-\mathrm{Si} 1$ & $120.19(3)$ \\
\hline 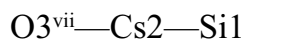 & $61.12(7)$ \\
\hline $\mathrm{Cs} 1-\mathrm{Cs} 2-\mathrm{Si} 1$ & $170.18(2)$ \\
\hline $\mathrm{O} 1-\mathrm{Cs} 2-\mathrm{Si} 1$ & $62.13(6)$ \\
\hline $\mathrm{Cs} 3^{\mathrm{vii}}-\mathrm{Cs} 2-\mathrm{Si} 1$ & $68.87(2)$ \\
\hline $\mathrm{Cs} 3-\mathrm{Cs} 2-\mathrm{Si} 1$ & $59.79(2)$ \\
\hline $\mathrm{Si} 2^{\mathrm{v}}-\mathrm{Cs} 2-\mathrm{Si} 1$ & $121.47(3)$ \\
\hline $\mathrm{O} 4^{\mathrm{iii}}-\mathrm{Cs} 2-\mathrm{Si} 1$ & $95.74(6)$ \\
\hline $\mathrm{O} 4 \mathrm{i}-\mathrm{Cs} 2-\mathrm{Si} 1$ & $128.22(6)$ \\
\hline $\mathrm{Cs} 1^{\mathrm{xiv}}-\mathrm{Cs} 2-\mathrm{Si} 1$ & $71.51(2)$ \\
\hline $\mathrm{Lu} 1^{\mathrm{ii}}-\mathrm{Cs} 2-\mathrm{Si} 1$ & $126.07(2)$ \\
\hline $\mathrm{O} 8^{\mathrm{v}}-\mathrm{Cs} 2-\mathrm{Si} 1$ & $98.40(6)$ \\
\hline $\mathrm{O} 9^{\text {iv }}-\mathrm{Cs} 2-\mathrm{Si} 1$ & $102.45(6)$ \\
\hline $\mathrm{Si} 1^{\mathrm{iv}}-\mathrm{Cs} 2-\mathrm{Si} 1$ & $107.75(3)$ \\
\hline $\mathrm{Lu} 1^{\mathrm{iii}}-\mathrm{Cs} 2-\mathrm{Si} 1$ & $91.76(2)$ \\
\hline $\mathrm{O} 2^{\mathrm{ii}}-\mathrm{Cs} 2-\mathrm{Si}^{2}{ }^{\mathrm{v}}$ & $60.35(8)$ \\
\hline
\end{tabular}

\begin{tabular}{|c|c|}
\hline $\mathrm{Cs} 3-\mathrm{O} 4-\mathrm{Cs}^{\mathrm{xv}}$ & $149.06(12)$ \\
\hline $\mathrm{Cs} 2^{\mathrm{x}}-\mathrm{O} 4-\mathrm{Cs} 1^{\mathrm{xv}}$ & $76.74(6)$ \\
\hline $\mathrm{Cs} 2^{\mathrm{ix}}-\mathrm{O} 4-\mathrm{Cs} 1^{\mathrm{xv}}$ & $51.25(5)$ \\
\hline $\mathrm{Cs} 3^{\mathrm{ix}}-\mathrm{O} 4-\mathrm{Cs} 1^{\mathrm{xv}}$ & $65.89(5)$ \\
\hline $\mathrm{Cs} 1^{\mathrm{x}}-\mathrm{O} 4-\mathrm{Cs} 1^{\mathrm{xv}}$ & $110.48(8)$ \\
\hline $\mathrm{Si} 2^{\mathrm{x}}-\mathrm{O} 4-\mathrm{Cs} 2^{\mathrm{xi}}$ & $87.39(16)$ \\
\hline $\mathrm{Lu} 1-\mathrm{O} 4-\mathrm{Cs} 2^{\mathrm{xi}}$ & $130.98(14)$ \\
\hline $\mathrm{Cs} 1^{\mathrm{ix}}-\mathrm{O} 4-\mathrm{Cs} 2^{\mathrm{xi}}$ & $51.96(6)$ \\
\hline $\mathrm{Cs} 3-\mathrm{O} 4-\mathrm{Cs} 2^{\mathrm{xi}}$ & $45.55(5)$ \\
\hline $\mathrm{Cs} 2^{\mathrm{x}}-\mathrm{O} 4-\mathrm{Cs} 2^{\mathrm{xi}}$ & $104.14(9)$ \\
\hline $\mathrm{Cs} 2^{\mathrm{ix}}-\mathrm{O} 4-\mathrm{Cs} 2^{\mathrm{xi}}$ & $107.65(8)$ \\
\hline $\mathrm{Cs} 3^{\mathrm{ix}}-\mathrm{O} 4-\mathrm{Cs} 2^{\mathrm{xi}}$ & $152.42(9)$ \\
\hline $\mathrm{Cs} 1^{\mathrm{x}}-\mathrm{O} 4-\mathrm{Cs} 2^{\mathrm{xi}}$ & $61.98(5)$ \\
\hline $\mathrm{Cs} 1^{\mathrm{xv}}-\mathrm{O} 4-\mathrm{Cs} 2^{\mathrm{xi}}$ & $113.36(7)$ \\
\hline Si1-O5-Lu1 & $137.8(3)$ \\
\hline $\mathrm{Si} 1-\mathrm{O} 5-\mathrm{Cs} 3$ & $122.8(2)$ \\
\hline $\mathrm{Lu} 1-\mathrm{O} 5-\mathrm{Cs} 3$ & $88.15(13)$ \\
\hline $\mathrm{Si} 1-\mathrm{O} 5-\mathrm{Cs} 2^{\mathrm{x}}$ & $96.62(19)$ \\
\hline $\mathrm{Lu} 1-\mathrm{O} 5-\mathrm{Cs} 2^{\mathrm{x}}$ & $104.55(16)$ \\
\hline $\mathrm{Cs} 3-\mathrm{O} 5-\mathrm{Cs} 2^{\mathrm{x}}$ & $102.11(13)$ \\
\hline $\mathrm{Si} 1-\mathrm{O} 5-\mathrm{Cs} 1^{\mathrm{x}}$ & $85.58(16)$ \\
\hline $\mathrm{Lu} 1-\mathrm{O} 5-\mathrm{Cs} 1^{\mathrm{x}}$ & $136.62(16)$ \\
\hline $\mathrm{Cs} 3-\mathrm{O} 5-\mathrm{Cs} 1^{\mathrm{x}}$ & $59.09(7)$ \\
\hline $\mathrm{Cs} 2^{\mathrm{x}}-\mathrm{O} 5-\mathrm{Cs} 1^{\mathrm{x}}$ & $61.39(7)$ \\
\hline $\mathrm{Si} 1-\mathrm{O} 5-\mathrm{Cs} 3^{\mathrm{vii}}$ & $59.13(14)$ \\
\hline $\mathrm{Lu} 1-\mathrm{O} 5-\mathrm{Cs} 3^{\mathrm{vii}}$ & $101.90(14)$ \\
\hline $\mathrm{Cs} 3-\mathrm{O} 5-\mathrm{Cs} 3^{\mathrm{vii}}$ & $160.28(14)$ \\
\hline $\mathrm{Cs} 2^{\mathrm{x}}-\mathrm{O} 5-\mathrm{Cs} 3^{\mathrm{vii}}$ & $59.13(7)$ \\
\hline $\mathrm{Cs} 1^{\mathrm{x}}-\mathrm{O} 5-\mathrm{Cs} 3^{\mathrm{vii}}$ & $103.28(9)$ \\
\hline $\mathrm{Si} 1-\mathrm{O} 5-\mathrm{Cs} 2$ & $77.40(17)$ \\
\hline $\mathrm{Lu} 1-\mathrm{O} 5-\mathrm{Cs} 2$ & $101.36(14)$ \\
\hline $\mathrm{Cs} 3-\mathrm{O} 5-\mathrm{Cs} 2$ & $57.86(7)$ \\
\hline $\mathrm{Cs} 2^{\mathrm{x}}-\mathrm{O} 5-\mathrm{Cs} 2$ & $146.59(12)$ \\
\hline $\mathrm{Cs} 11^{\mathrm{x}}-\mathrm{O} 5-\mathrm{Cs} 2$ & $85.27(8)$ \\
\hline $\mathrm{Cs} 3^{\mathrm{vii}}-\mathrm{O} 5-\mathrm{Cs} 2$ & $134.35(10)$ \\
\hline $\mathrm{Si} 1-\mathrm{O} 5-\mathrm{Cs} 1^{\mathrm{xiv}}$ & $91.53(18)$ \\
\hline $\mathrm{Lu} 1-\mathrm{O} 5-\mathrm{Cs}^{1 \mathrm{xiv}}$ & $57.94(10)$ \\
\hline $\mathrm{Cs} 3-\mathrm{O} 5-\mathrm{Cs} 1^{\mathrm{xiv}}$ & $89.39(10)$ \\
\hline $\mathrm{Cs} 2^{\mathrm{x}}-\mathrm{O} 5-\mathrm{Cs} 1^{\mathrm{xiv}}$ & $159.11(13)$ \\
\hline $\mathrm{Cs} 1^{\mathrm{x}}-\mathrm{O} 5-\mathrm{Cs} 1^{\mathrm{xiv}}$ & $138.73(11)$ \\
\hline $\mathrm{Cs} 3^{\mathrm{vii}}-\mathrm{O} 5-\mathrm{Cs} 1^{\mathrm{xiv}}$ & $110.33(9)$ \\
\hline $\mathrm{Cs} 2-\mathrm{O} 5-\mathrm{Cs} 1^{\mathrm{xiv}}$ & $54.11(5)$ \\
\hline $\mathrm{Si} 1-\mathrm{O} 5-\mathrm{Cs} 1^{\mathrm{xii}}$ & $92.82(17)$ \\
\hline $\mathrm{Lu} 1-\mathrm{O} 5-\mathrm{Cs} 1^{\mathrm{xii}}$ & $57.75(9)$ \\
\hline $\mathrm{Cs} 3-\mathrm{O} 5-\mathrm{Cs}^{1 \mathrm{xi}}$ & $143.79(12)$ \\
\hline $\mathrm{Cs} 2^{\mathrm{x}}-\mathrm{O} 5-\mathrm{Cs} 1^{\mathrm{xii}}$ & $77.86(9)$ \\
\hline $\mathrm{Cs} 1^{\mathrm{x}}-\mathrm{O} 5-\mathrm{Cs} 1^{\mathrm{xii}}$ & $138.65(11)$ \\
\hline $\mathrm{Cs} 3^{\mathrm{vii}}-\mathrm{O} 5-\mathrm{Cs} 1^{\mathrm{xii}}$ & $44.34(4)$ \\
\hline
\end{tabular}




\begin{tabular}{|c|c|}
\hline  & $116.15(8)$ \\
\hline $\mathrm{O} 6-\mathrm{Cs} 2-\mathrm{Si}^{\mathrm{v}}$ & $96.95(8)$ \\
\hline $\mathrm{O} 8-\mathrm{Cs} 2-\mathrm{Si}^{\mathrm{v}}$ & $108.11(7)$ \\
\hline $\mathrm{O}^{\mathrm{iii}}-\mathrm{Cs} 2-\mathrm{Si}^{\mathrm{v}}$ & $159.88(8)$ \\
\hline $\mathrm{O} 7-\mathrm{Cs} 2-\mathrm{Si}^{\mathrm{v}}$ & $99.75(8)$ \\
\hline $\mathrm{Si} 3-\mathrm{Cs} 2-\mathrm{Si}^{\mathrm{v}}$ & $118.10(3)$ \\
\hline $\mathrm{Si} 2-\mathrm{Cs} 2-\mathrm{Si}^{\mathrm{v}}$ & $115.15(3)$ \\
\hline 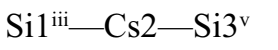 & $139.86(3)$ \\
\hline $\mathrm{O} 3^{\mathrm{vii}}-\mathrm{Cs} 2-\mathrm{Si}^{\mathrm{v}}$ & $142.10(7)$ \\
\hline $\mathrm{Cs} 1-\mathrm{Cs} 2-\mathrm{Si}^{\mathrm{v}}$ & $90.37(2)$ \\
\hline $\mathrm{O} 1-\mathrm{Cs} 2-\mathrm{Si}^{\mathrm{v}}$ & $20.57(6)$ \\
\hline $\mathrm{Cs} 3^{\mathrm{viii}}-\mathrm{Cs} 2-\mathrm{Si} 3^{\mathrm{v}}$ & $107.82(2)$ \\
\hline $\mathrm{Cs} 3-\mathrm{Cs} 2-\mathrm{Si}^{\mathrm{v}}$ & $52.07(2)$ \\
\hline $\mathrm{Si} 2^{v}-\mathrm{Cs} 2-\mathrm{Si}^{\mathrm{v}}{ }^{\mathrm{v}}$ & $42.05(3)$ \\
\hline $\mathrm{O} 4^{\mathrm{iii}}-\mathrm{Cs} 2-\mathrm{Si}^{\mathrm{v}}$ & $137.98(6)$ \\
\hline $\mathrm{O} 4^{\mathrm{i}}-\mathrm{Cs} 2-\mathrm{Si}^{\mathrm{v}}$ & $46.50(6)$ \\
\hline $\mathrm{Cs} 1^{\mathrm{xiv}}-\mathrm{Cs} 2-\mathrm{Si}^{\mathrm{v}}$ & $48.76(2)$ \\
\hline $\mathrm{Lu} 1^{\mathrm{ii}}-\mathrm{Cs} 2-\mathrm{Si} 3^{\mathrm{v}}$ & $86.82(2)$ \\
\hline $\mathrm{O} 8^{\mathrm{v}}-\mathrm{Cs} 2-\mathrm{Si}^{\mathrm{v}}$ & $21.86(6)$ \\
\hline $\mathrm{O}^{\mathrm{iv}}-\mathrm{Cs} 2-\mathrm{Si}^{\mathrm{v}}$ & $58.92(6)$ \\
\hline $\mathrm{Si} 1^{\mathrm{iv}}-\mathrm{Cs} 2-\mathrm{Si}^{\mathrm{v}}{ }^{\mathrm{V}}$ & $80.59(3)$ \\
\hline $\mathrm{Lu} 1^{\mathrm{iii}}-\mathrm{Cs} 2-\mathrm{Si}^{\mathrm{v}}$ & $167.27(2)$ \\
\hline $\mathrm{Si} 1-\mathrm{Cs} 2-\mathrm{Si}^{\mathrm{v}}$ & $82.63(3)$ \\
\hline $\mathrm{O} 1-\mathrm{Cs} 3-\mathrm{O}^{\mathrm{iv}}$ & $100.78(11)$ \\
\hline $\mathrm{O} 1-\mathrm{Cs} 3-\mathrm{O} 5$ & $63.24(11)$ \\
\hline $\mathrm{O} 9^{\mathrm{iv}}-\mathrm{Cs} 3-\mathrm{O} 5$ & $154.42(12)$ \\
\hline $\mathrm{O} 1-\mathrm{Cs} 3-\mathrm{O} 4$ & $63.56(11)$ \\
\hline $\mathrm{O} 9^{\mathrm{iv}}-\mathrm{Cs} 3-\mathrm{O} 4$ & $131.81(11)$ \\
\hline $\mathrm{O} 5-\mathrm{Cs} 3-\mathrm{O} 4$ & $61.42(11)$ \\
\hline $\mathrm{O} 1-\mathrm{Cs} 3-\mathrm{O} 3^{\mathrm{ix}}$ & $50.44(10)$ \\
\hline $\mathrm{O} 9^{\mathrm{iv}}-\mathrm{Cs} 3-\mathrm{O} 3^{\mathrm{ix}}$ & $58.46(11)$ \\
\hline $\mathrm{O} 5-\mathrm{Cs} 3-\mathrm{O} 3^{\mathrm{ix}}$ & $112.92(10)$ \\
\hline $\mathrm{O} 4-\mathrm{Cs} 3-\mathrm{O} 3^{\mathrm{ix}}$ & $80.24(11)$ \\
\hline $\mathrm{O} 1-\mathrm{Cs} 3-\mathrm{O} 8$ & $111.75(11)$ \\
\hline $\mathrm{O} 9^{\mathrm{iv}}-\mathrm{Cs} 3-\mathrm{O} 8$ & $89.80(10)$ \\
\hline $\mathrm{O} 5-\mathrm{Cs} 3-\mathrm{O} 8$ & $79.16(10)$ \\
\hline $\mathrm{O} 4-\mathrm{Cs} 3-\mathrm{O} 8$ & $138.25(10)$ \\
\hline $\mathrm{O} 3^{\mathrm{ix}}-\mathrm{Cs} 3-\mathrm{O} 8$ & $131.18(10)$ \\
\hline $\mathrm{O} 1-\mathrm{Cs} 3-\mathrm{O} 6$ & $111.83(10)$ \\
\hline $\mathrm{O} 9^{\mathrm{iv}}-\mathrm{Cs} 3-\mathrm{O} 6$ & $46.36(10)$ \\
\hline $\mathrm{O} 5-\mathrm{Cs} 3-\mathrm{O} 6$ & $118.27(10)$ \\
\hline $\mathrm{O} 4-\mathrm{Cs} 3-\mathrm{O} 6$ & $175.22(10)$ \\
\hline $\mathrm{O} 3^{\mathrm{ix}}-\mathrm{Cs} 3-\mathrm{O} 6$ & $95.85(9)$ \\
\hline $\mathrm{O} 8-\mathrm{Cs} 3-\mathrm{O} 6$ & $43.48(9)$ \\
\hline $\mathrm{O} 1-\mathrm{Cs} 3-\mathrm{Cs}^{\mathrm{x}}$ & $137.68(8)$ \\
\hline $\mathrm{O} 9^{\mathrm{iv}}-\mathrm{Cs} 3-\mathrm{Cs} 1^{\mathrm{x}}$ & $116.05(8)$ \\
\hline $\mathrm{O} 5-\mathrm{Cs} 3-\mathrm{Cs} 1^{\mathrm{x}}$ & $74.79(8)$ \\
\hline $\mathrm{O} 4-\mathrm{Cs} 3-\mathrm{Cs} 1^{\mathrm{x}}$ & $101.55(8)$ \\
\hline
\end{tabular}

\begin{tabular}{|c|c|}
\hline $\mathrm{Cs} 2-\mathrm{O} 5-\mathrm{Cs} 1^{\mathrm{xii}}$ & $134.69(10)$ \\
\hline $\mathrm{Cs} 1^{\mathrm{xiv}}-\mathrm{O} 5-\mathrm{Cs} 1^{\mathrm{xii}}$ & $82.57(6)$ \\
\hline $\mathrm{Si} 2-\mathrm{O} 6-\mathrm{Si}^{\mathrm{iv}}$ & $135.8(2)$ \\
\hline $\mathrm{Si} 2-\mathrm{O} 6-\mathrm{Cs} 2$ & $94.35(17)$ \\
\hline $\mathrm{Si} 1^{\mathrm{iv}}-\mathrm{O} 6-\mathrm{Cs} 2$ & $125.96(19)$ \\
\hline $\mathrm{Si} 2-\mathrm{O} 6-\mathrm{Cs} 3$ & $97.1(2)$ \\
\hline $\mathrm{Si} 1^{\mathrm{iv}}-\mathrm{O} 6-\mathrm{Cs} 3$ & $83.32(18)$ \\
\hline $\mathrm{Cs} 2-\mathrm{O} 6-\mathrm{Cs} 3$ & $69.46(10)$ \\
\hline $\mathrm{Si} 2-\mathrm{O} 6-\mathrm{Cs} 3^{3 \mathrm{ii}}$ & $86.44(18)$ \\
\hline $\mathrm{Si}^{\mathrm{iv}}-\mathrm{O} 6-\mathrm{Cs} 3^{\mathrm{iii}}$ & $110.2(2)$ \\
\hline $\mathrm{Cs} 2-\mathrm{O} 6-\mathrm{Cs} 3^{\mathrm{iii}}$ & $87.13(9)$ \\
\hline $\mathrm{Cs} 3-\mathrm{O} 6-\mathrm{Cs} 3^{\mathrm{iii}}$ & $156.51(11)$ \\
\hline $\mathrm{Si} 2-\mathrm{O} 6-\mathrm{Cs} 1$ & $132.60(19)$ \\
\hline $\mathrm{Si}^{\mathrm{iv}}-\mathrm{O} 6-\mathrm{Cs} 1$ & $86.99(16)$ \\
\hline $\mathrm{Cs} 2-\mathrm{O} 6-\mathrm{Cs} 1$ & $61.75(6)$ \\
\hline $\mathrm{Cs} 3-\mathrm{O} 6-\mathrm{Cs} 1$ & $109.44(10)$ \\
\hline $\mathrm{Cs} 3^{\mathrm{iii}}-\mathrm{O} 6-\mathrm{Cs} 1$ & $54.45(6)$ \\
\hline $\mathrm{Si} 2-\mathrm{O} 6-\mathrm{Cs} 1^{\mathrm{xiii}}$ & $49.41(14)$ \\
\hline $\mathrm{Si} 1^{\mathrm{iv}}-\mathrm{O} 6-\mathrm{Cs} 1^{\mathrm{xiii}}$ & $104.59(18)$ \\
\hline Cs2-O6-Cs $1^{\text {xiii }}$ & $126.75(11)$ \\
\hline $\mathrm{Cs} 3-\mathrm{O} 6-\mathrm{Cs} 1^{\mathrm{xiii}}$ & $139.09(11)$ \\
\hline $\mathrm{Cs} 3^{\mathrm{iii}}-\mathrm{O} 6-\mathrm{Cs} 1^{\mathrm{xiii}}$ & $57.90(7)$ \\
\hline Cs1-O6-Cs1 $1^{\mathrm{xiii}}$ & $111.00(11)$ \\
\hline $\mathrm{Si} 2-\mathrm{O} 6-\mathrm{Cs} 1^{\mathrm{x}}$ & $51.55(15)$ \\
\hline $\mathrm{Si}^{\mathrm{iv}}-\mathrm{O} 6-\mathrm{Cs}^{\mathrm{x}}$ & $104.07(18)$ \\
\hline $\mathrm{Cs} 2-\mathrm{O} 6-\mathrm{Cs} 1^{\mathrm{x}}$ & $92.17(11)$ \\
\hline $\mathrm{Cs} 3-\mathrm{O} 6-\mathrm{Cs} 1^{\mathrm{x}}$ & $49.00(6)$ \\
\hline 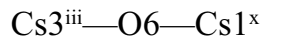 & $137.84(9)$ \\
\hline $\mathrm{Cs} 1-\mathrm{O} 6-\mathrm{Cs} 1^{\mathrm{x}}$ & $152.70(12)$ \\
\hline $\mathrm{Cs} 1^{\mathrm{xiii}}-\mathrm{O} 6-\mathrm{Cs} 1^{\mathrm{x}}$ & $90.58(7)$ \\
\hline $\mathrm{Si} 2-\mathrm{O} 6-\mathrm{Cs} 2^{\mathrm{iv}}$ & $103.2(2)$ \\
\hline $\mathrm{Si} 1^{\mathrm{iv}}-\mathrm{O} 6-\mathrm{Cs} 2^{\mathrm{iv}}$ & $64.81(17)$ \\
\hline $\mathrm{Cs} 2-\mathrm{O} 6-\mathrm{Cs} 2^{\mathrm{iv}}$ & $132.16(13)$ \\
\hline $\mathrm{Cs} 3-\mathrm{O} 6-\mathrm{Cs} 2^{\mathrm{iv}}$ & $147.93(10)$ \\
\hline $\mathrm{Cs} 3^{3 i i}-\mathrm{O} 6-\mathrm{Cs} 2^{\mathrm{iv}}$ & $50.83(6)$ \\
\hline $\mathrm{Cs} 1-\mathrm{O} 6-\mathrm{Cs} 2^{\mathrm{iv}}$ & $73.98(8)$ \\
\hline $\mathrm{Cs} 1^{\mathrm{xiii}}-\mathrm{O} 6-\mathrm{Cs} 2^{\mathrm{iv}}$ & $53.83(6)$ \\
\hline $\mathrm{Cs} 1^{\mathrm{x}}-\mathrm{O} 6-\mathrm{Cs} 2^{\mathrm{iv}}$ & $133.32(8)$ \\
\hline $\mathrm{Si} 2-\mathrm{O} 6-\mathrm{Cs} 2^{\mathrm{xi}}$ & $113.05(18)$ \\
\hline $\mathrm{Si}^{\mathrm{iv}}-\mathrm{O} 6-\mathrm{Cs} 2^{\mathrm{xi}}$ & $37.54(14)$ \\
\hline $\mathrm{Cs} 2-\mathrm{O} 6-\mathrm{Cs} 2^{\mathrm{xi}}$ & $115.84(12)$ \\
\hline $\mathrm{Cs} 3-\mathrm{O} 6-\mathrm{Cs} 2^{\mathrm{xi}}$ & $51.19(5)$ \\
\hline $\mathrm{Cs} 3^{\mathrm{iii}}-\mathrm{O} 6-\mathrm{Cs} 2^{\mathrm{xi}}$ & $147.15(11)$ \\
\hline $\mathrm{Cs} 1-\mathrm{O} 6-\mathrm{Cs} 2^{\mathrm{xi}}$ & $114.21(8)$ \\
\hline $\mathrm{Cs} 1^{\mathrm{xii}}-\mathrm{O} 6-\mathrm{Cs} 2^{\mathrm{xi}}$ & $114.35(8)$ \\
\hline $\mathrm{Cs} 1^{\mathrm{x}}-\mathrm{O} 6-\mathrm{Cs} 2^{\mathrm{xi}}$ & $68.09(6)$ \\
\hline $\mathrm{Cs} 2^{\mathrm{iv}}-\mathrm{O} 6-\mathrm{Cs} 2^{\mathrm{xi}}$ & $97.58(7)$ \\
\hline $\mathrm{Si} 2-\mathrm{O} 6-\mathrm{Cs} 3^{\mathrm{iv}}$ & $105.48(16)$ \\
\hline
\end{tabular}




\begin{tabular}{|c|c|}
\hline $\mathrm{O} 3^{\mathrm{ix}}-\mathrm{Cs} 3-\mathrm{Cs} 1^{\mathrm{x}}$ & $171.62(7)$ \\
\hline $\mathrm{O} 8-\mathrm{Cs} 3-\mathrm{Cs} 1^{\mathrm{x}}$ & $51.87(7)$ \\
\hline $\mathrm{O} 6-\mathrm{Cs} 3-\mathrm{Cs} 1^{\mathrm{x}}$ & $82.71(6)$ \\
\hline $\mathrm{O} 1-\mathrm{Cs} 3-\mathrm{Si}^{\mathrm{v}}$ & $24.90(8)$ \\
\hline $\mathrm{O} 9^{\mathrm{iv}}-\mathrm{Cs} 3-\mathrm{Si}^{\mathrm{v}}$ & $78.98(8)$ \\
\hline $\mathrm{O} 5-\mathrm{Cs} 3-\mathrm{Si}^{\mathrm{v}}$ & $87.92(8)$ \\
\hline $\mathrm{O} 4-\mathrm{Cs} 3-\mathrm{Si}^{\mathrm{v}}$ & $71.10(9)$ \\
\hline $\mathrm{O}^{\mathrm{ix}}-\mathrm{Cs} 3-\mathrm{Si}^{\mathrm{v}}$ & $25.59(7)$ \\
\hline $\mathrm{O} 8-\mathrm{Cs} 3-\mathrm{Si}^{\mathrm{v}}$ & $123.47(7)$ \\
\hline $\mathrm{O} 6-\mathrm{Cs} 3-\mathrm{Si}^{\mathrm{v}}$ & $104.20(7)$ \\
\hline $\mathrm{Cs}^{\mathrm{x}}-\mathrm{Cs} 3-\mathrm{Si}^{\mathrm{v}}$ & $162.58(3)$ \\
\hline $\mathrm{O} 1-\mathrm{Cs} 3-\mathrm{Lu} 1$ & $37.14(8)$ \\
\hline $\mathrm{O} 9^{\mathrm{iv}}-\mathrm{Cs} 3-\mathrm{Lu} 1$ & $136.42(8)$ \\
\hline $\mathrm{O} 5-\mathrm{Cs} 3-\mathrm{Lu} 1$ & $36.96(7)$ \\
\hline $\mathrm{O} 4-\mathrm{Cs} 3-\mathrm{Lu} 1$ & $36.76(7)$ \\
\hline $\mathrm{O} 3^{\mathrm{ix}}-\mathrm{Cs} 3-\mathrm{Lu} 1$ & $79.34(7)$ \\
\hline $\mathrm{O} 8-\mathrm{Cs} 3-\mathrm{Lu} 1$ & $113.09(7)$ \\
\hline $\mathrm{O} 6-\mathrm{Cs} 3-\mathrm{Lu} 1$ & $140.02(6)$ \\
\hline $\mathrm{Cs} 1^{x}-\mathrm{Cs} 3-\mathrm{Lu} 1$ & $107.062(14)$ \\
\hline $\mathrm{Si} 3^{\mathrm{v}}-\mathrm{Cs} 3-\mathrm{Lu} 1$ & $57.44(2)$ \\
\hline $\mathrm{O} 1-\mathrm{Cs} 3-\mathrm{Si}^{\mathrm{iv}}$ & $116.51(8)$ \\
\hline $\mathrm{O} 9^{\mathrm{iv}}-\mathrm{Cs} 3-\mathrm{Si}^{\mathrm{iv}}$ & $23.97(8)$ \\
\hline $\mathrm{O} 5-\mathrm{Cs} 3-\mathrm{Si}^{\mathrm{iv}}$ & $144.07(9)$ \\
\hline $\mathrm{O} 4-\mathrm{Cs} 3-\mathrm{Si}^{\mathrm{iv}}$ & $153.85(8)$ \\
\hline $\mathrm{O} 3^{\mathrm{ix}}-\mathrm{Cs} 3-\mathrm{Si}^{\mathrm{iv}}$ & $81.49(7)$ \\
\hline $\mathrm{O} 8-\mathrm{Cs} 3-\mathrm{Si}^{\mathrm{iv}}$ & $67.60(7)$ \\
\hline $\mathrm{O} 6-\mathrm{Cs} 3-\mathrm{Si}^{\mathrm{iv}}$ & $25.86(6)$ \\
\hline $\mathrm{Cs} 1^{\mathrm{x}}-\mathrm{Cs} 3-\mathrm{Si}^{\mathrm{iv}}$ & $94.03(2)$ \\
\hline $\mathrm{Si} 3^{\mathrm{v}}-\mathrm{Cs} 3-\mathrm{Si}^{\mathrm{iv}}$ & $98.92(3)$ \\
\hline $\mathrm{Lu} 1-\mathrm{Cs} 3-\mathrm{Si}^{\mathrm{iv}}$ & $153.38(3)$ \\
\hline $\mathrm{O} 1-\mathrm{Cs} 3-\mathrm{Cs} 2^{\mathrm{xi}}$ & $127.70(8)$ \\
\hline $\mathrm{O} 9^{\mathrm{iv}}-\mathrm{Cs} 3-\mathrm{Cs} 2^{\mathrm{xi}}$ & $52.01(9)$ \\
\hline $\mathrm{O} 5-\mathrm{Cs} 3-\mathrm{Cs} 2^{\mathrm{xi}}$ & $153.55(9)$ \\
\hline $\mathrm{O} 4-\mathrm{Cs} 3-\mathrm{Cs} 2^{\mathrm{xi}}$ & $99.79(7)$ \\
\hline $\mathrm{O} 3^{\mathrm{ix}}-\mathrm{Cs} 3-\mathrm{Cs} 2^{\mathrm{xi}}$ & $78.98(7)$ \\
\hline $\mathrm{O} 8-\mathrm{Cs} 3-\mathrm{Cs} 2^{\mathrm{xi}}$ & $111.58(7)$ \\
\hline $\mathrm{O} 6-\mathrm{Cs} 3-\mathrm{Cs} 2^{\mathrm{xi}}$ & $82.05(6)$ \\
\hline $\mathrm{Cs} 1^{\mathrm{x}}-\mathrm{Cs} 3-\mathrm{Cs} 2^{\mathrm{xi}}$ & $92.647(13)$ \\
\hline $\mathrm{Si} 3^{\mathrm{v}}-\mathrm{Cs} 3-\mathrm{Cs} 2^{\mathrm{xi}}$ & $104.05(3)$ \\
\hline $\mathrm{Lu} 1-\mathrm{Cs} 3-\mathrm{Cs} 2^{\mathrm{xi}}$ & $134.225(14)$ \\
\hline $\mathrm{Si} 1^{\mathrm{iv}}-\mathrm{Cs} 3-\mathrm{Cs} 2^{\mathrm{xi}}$ & $58.23(2)$ \\
\hline $\mathrm{O} 1-\mathrm{Cs} 3-\mathrm{Cs} 2$ & $67.00(8)$ \\
\hline $\mathrm{O} 9^{\mathrm{iv}}-\mathrm{Cs} 3-\mathrm{Cs} 2$ & $75.21(9)$ \\
\hline $\mathrm{O} 5-\mathrm{Cs} 3-\mathrm{Cs} 2$ & $79.94(9)$ \\
\hline $\mathrm{O} 4-\mathrm{Cs} 3-\mathrm{Cs} 2$ & $126.81(8)$ \\
\hline $\mathrm{O} 3^{\mathrm{ix}}-\mathrm{Cs} 3-\mathrm{Cs} 2$ & $83.30(8)$ \\
\hline $\mathrm{O} 8-\mathrm{Cs} 3-\mathrm{Cs} 2$ & $51.14(7)$ \\
\hline $\mathrm{O} 6-\mathrm{Cs} 3-\mathrm{Cs} 2$ & $49.52(6)$ \\
\hline
\end{tabular}

\begin{tabular}{|c|c|}
\hline $\mathrm{Si} 1^{\mathrm{iv}}-\mathrm{O} 6-\mathrm{Cs} 3^{\mathrm{iv}}$ & $35.56(12)$ \\
\hline $\mathrm{Cs} 2-\mathrm{O} 6-\mathrm{Cs} 3^{\mathrm{iv}}$ & $160.16(11)$ \\
\hline $\mathrm{Cs} 3-\mathrm{O} 6-\mathrm{Cs} 3^{\mathrm{iv}}$ & $106.91(8)$ \\
\hline $\mathrm{Cs} 3^{\mathrm{iii}}-\mathrm{O} 6-\mathrm{Cs} 3^{\mathrm{iv}}$ & $94.37(9)$ \\
\hline $\mathrm{Cs} 1-\mathrm{O} 6-\mathrm{Cs}^{\mathrm{iv}}$ & $103.37(9)$ \\
\hline $\mathrm{Cs} 1^{\mathrm{xiii}}-\mathrm{O} 6-\mathrm{Cs} 3^{\mathrm{iv}}$ & $69.30(6)$ \\
\hline $\mathrm{Cs} 1^{\mathrm{x}}-\mathrm{O} 6-\mathrm{Cs} 3^{\mathrm{iv}}$ & $99.77(7)$ \\
\hline $\mathrm{Cs} 2^{\mathrm{iv}}-\mathrm{O} 6-\mathrm{Cs} 3^{\mathrm{iv}}$ & $43.78(4)$ \\
\hline $\mathrm{Cs} 2^{\mathrm{xi}}-\mathrm{O} 6-\mathrm{Cs} 3^{\mathrm{iv}}$ & $55.96(4)$ \\
\hline $\mathrm{Si} 2-\mathrm{O} 6-\mathrm{Cs} 2^{\mathrm{xiii}}$ & $17.22(10)$ \\
\hline $\mathrm{Si} 1^{\mathrm{iv}}-\mathrm{O} 6-\mathrm{Cs} 2^{\mathrm{xiii}}$ & $118.63(16)$ \\
\hline $\mathrm{Cs} 2-\mathrm{O} 6-\mathrm{Cs} 2^{\mathrm{xiii}}$ & $110.49(10)$ \\
\hline $\mathrm{Cs} 3-\mathrm{O} 6-\mathrm{Cs} 2^{\mathrm{xiii}}$ & $96.83(9)$ \\
\hline $\mathrm{Cs} 3^{3 i i}-\mathrm{O} 6-\mathrm{Cs} 2^{\mathrm{xiii}}$ & $93.24(8)$ \\
\hline $\mathrm{Cs} 1-\mathrm{O} 6-\mathrm{Cs} 2^{\mathrm{xiii}}$ & $145.60(10)$ \\
\hline $\mathrm{Cs} 1^{\mathrm{xiii}}-\mathrm{O} 6-\mathrm{Cs} 2^{\mathrm{xiii}}$ & $43.78(3)$ \\
\hline $\mathrm{Cs} 1^{\mathrm{x}}-\mathrm{O} 6-\mathrm{Cs} 2^{\mathrm{xiii}}$ & $47.91(4)$ \\
\hline $\mathrm{Cs} 2^{\mathrm{iv}}-\mathrm{O} 6-\mathrm{Cs} 2^{\mathrm{xiii}}$ & $95.49(7)$ \\
\hline $\mathrm{Cs} 2^{\mathrm{xi}}-\mathrm{O} 6-\mathrm{Cs} 2^{\mathrm{xiii}}$ & $99.44(7)$ \\
\hline $\mathrm{Cs} 3^{\text {iv }}-\mathrm{O} 6-\mathrm{Cs} 2^{\text {xiii }}$ & $89.20(6)$ \\
\hline $\mathrm{Si} 3-\mathrm{O} 7-\mathrm{Si} 1$ & $133.0(3)$ \\
\hline $\mathrm{Si} 3-\mathrm{O} 7-\mathrm{Cs} 2$ & $90.35(17)$ \\
\hline $\mathrm{Si} 1-\mathrm{O} 7-\mathrm{Cs} 2$ & $126.6(2)$ \\
\hline $\mathrm{Si} 3-\mathrm{O} 7-\mathrm{Cs} 1^{\mathrm{vii}}$ & $79.85(16)$ \\
\hline $\mathrm{Si} 1-\mathrm{O} 7-\mathrm{Cs} 1^{\mathrm{vii}}$ & $109.26(19)$ \\
\hline $\mathrm{Cs} 2-\mathrm{O} 7-\mathrm{Cs} 1^{\mathrm{vii}}$ & $107.70(11)$ \\
\hline $\mathrm{Si} 3-\mathrm{O} 7-\mathrm{Cs} 3^{\text {viii }}$ & $116.20(18)$ \\
\hline $\mathrm{Si} 1-\mathrm{O} 7-\mathrm{Cs} 3^{\text {viii }}$ & $106.73(17)$ \\
\hline $\mathrm{Cs} 2-\mathrm{O} 7-\mathrm{Cs} 3^{\mathrm{viii}}$ & $64.09(7)$ \\
\hline $\mathrm{Cs} 1^{\mathrm{vii}}-\mathrm{O} 7-\mathrm{Cs} 3^{\mathrm{viii}}$ & $58.27(6)$ \\
\hline $\mathrm{Si} 3-\mathrm{O} 7-\mathrm{Cs} 3$ & $92.68(16)$ \\
\hline $\mathrm{Si} 1-\mathrm{O} 7-\mathrm{Cs} 3$ & $82.41(16)$ \\
\hline $\mathrm{Cs} 2-\mathrm{O} 7-\mathrm{Cs} 3$ & $63.04(7)$ \\
\hline $\mathrm{Cs} 1{ }^{\mathrm{vii}}-\mathrm{O} 7-\mathrm{Cs} 3$ & $168.32(12)$ \\
\hline $\mathrm{Cs} 3^{\text {viii }}-\mathrm{O} 7-\mathrm{Cs} 3$ & $118.79(10)$ \\
\hline $\mathrm{Si} 3-\mathrm{O} 7-\mathrm{Cs} 1^{\mathrm{x}}$ & $58.58(13)$ \\
\hline $\mathrm{Si} 1-\mathrm{O} 7-\mathrm{Cs} 1^{\mathrm{x}}$ & $82.85(15)$ \\
\hline $\mathrm{Cs} 2-\mathrm{O} 7-\mathrm{Cs} 1^{\mathrm{x}}$ & $103.29(10)$ \\
\hline $\mathrm{Cs} 1^{\mathrm{vii}}-\mathrm{O} 7-\mathrm{Cs} 1^{\mathrm{x}}$ & $127.58(11)$ \\
\hline $\mathrm{Cs} 3^{\mathrm{viii}}-\mathrm{O} 7-\mathrm{Cs} 1^{\mathrm{x}}$ & $167.06(12)$ \\
\hline $\mathrm{Cs} 3-\mathrm{O} 7-\mathrm{Cs} 1^{\mathrm{x}}$ & $52.75(5)$ \\
\hline $\mathrm{Si} 3-\mathrm{O} 7-\mathrm{Cs} 2^{\mathrm{vii}}$ & $121.83(18)$ \\
\hline $\mathrm{Si} 1-\mathrm{O} 7-\mathrm{Cs} 2^{\mathrm{vii}}$ & $60.47(14)$ \\
\hline $\mathrm{Cs} 2-\mathrm{O} 7-\mathrm{Cs} 2^{\mathrm{vii}}$ & $128.36(10)$ \\
\hline $\mathrm{Cs} 1^{\mathrm{vii}}-\mathrm{O} 7-\mathrm{Cs} 2^{\mathrm{vii}}$ & $50.48(5)$ \\
\hline  & $65.49(6)$ \\
\hline $\mathrm{Cs} 3-\mathrm{O} 7-\mathrm{Cs} 2^{\mathrm{vii}}$ & $140.49(10)$ \\
\hline $\mathrm{Cs} 1^{\mathrm{x}}-\mathrm{O} 7-\mathrm{Cs} 2^{\mathrm{vii}}$ & $127.45(9)$ \\
\hline
\end{tabular}




\begin{tabular}{|c|c|c|c|}
\hline $\mathrm{Cs} 1^{\mathrm{x}}-\mathrm{Cs} 3-\mathrm{Cs} 2$ & $101.762(14)$ & $\mathrm{Si} 3-\mathrm{O} 7-\mathrm{Cs} 3^{\mathrm{xiii}}$ & $33.75(12)$ \\
\hline $\mathrm{Si} 3^{\mathrm{v}}-\mathrm{Cs} 3-\mathrm{Cs} 2$ & $72.51(2)$ & $\mathrm{Si} 1-\mathrm{O} 7-\mathrm{Cs} 3^{\mathrm{xiii}}$ & $116.53(18)$ \\
\hline $\mathrm{Lu} 1-\mathrm{Cs} 3-\mathrm{Cs} 2$ & $90.584(12)$ & $\mathrm{Cs} 2-\mathrm{O} 7-\mathrm{Cs} 3^{\mathrm{xiii}}$ & $116.31(10)$ \\
\hline $\mathrm{Si} 1^{\mathrm{iv}}-\mathrm{Cs} 3-\mathrm{Cs} 2$ & $68.89(2)$ & $\mathrm{Cs} 1^{\mathrm{vii}}-\mathrm{O} 7-\mathrm{Cs} 3^{\mathrm{xiii}}$ & $53.19(6)$ \\
\hline $\mathrm{Cs} 2^{\mathrm{xi}}-\mathrm{Cs} 3-\mathrm{Cs} 2$ & $125.955(16)$ & 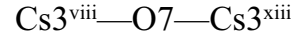 & $106.82(9)$ \\
\hline $\mathrm{O} 1-\mathrm{Cs} 3-\mathrm{O} 6^{\mathrm{x}}$ & $106.20(10)$ & $\mathrm{Cs} 3-\mathrm{O} 7-\mathrm{Cs} 3^{\mathrm{xiii}}$ & $122.78(9)$ \\
\hline $\mathrm{O} 9^{\mathrm{iv}}-\mathrm{Cs} 3-\mathrm{O}^{\mathrm{x}}$ & $129.89(11)$ & $\mathrm{Cs} 1^{\mathrm{x}}-\mathrm{O} 7-\mathrm{Cs} 3^{\mathrm{xiii}}$ & $75.41(6)$ \\
\hline $\mathrm{O} 5-\mathrm{Cs} 3-\mathrm{O}^{\mathrm{x}}$ & $75.42(11)$ & $\mathrm{Cs} 2^{\mathrm{vii}}-\mathrm{O} 7-\mathrm{Cs} 3^{\mathrm{xiii}}$ & $88.17(7)$ \\
\hline $\mathrm{O} 4-\mathrm{Cs} 3-\mathrm{O}^{\mathrm{x}}$ & $43.01(10)$ & $\mathrm{Si} 3-\mathrm{O} 7-\mathrm{Cs} 2^{\mathrm{x}}$ & $101.95(16)$ \\
\hline $\mathrm{O} 3^{\mathrm{ix}}-\mathrm{Cs} 3-\mathrm{O} 6^{\mathrm{x}}$ & $111.89(9)$ & $\mathrm{Si} 1-\mathrm{O} 7-\mathrm{Cs} 2^{\mathrm{x}}$ & $34.70(11)$ \\
\hline $\mathrm{O} 8-\mathrm{Cs} 3-\mathrm{O}^{\mathrm{x}}$ & $116.91(9)$ & $\mathrm{Cs} 2-\mathrm{O} 7-\mathrm{Cs} 2^{\mathrm{x}}$ & $126.23(11)$ \\
\hline $\mathrm{O} 6-\mathrm{Cs} 3-\mathrm{O}^{\mathrm{x}}$ & $141.73(6)$ &  & $125.89(10)$ \\
\hline $\mathrm{Cs} 1^{\mathrm{x}}-\mathrm{Cs} 3-\mathrm{O} 6^{\mathrm{x}}$ & $65.96(6)$ & $\mathrm{Cs} 3^{\mathrm{viii}}-\mathrm{O} 7-\mathrm{Cs} 2^{\mathrm{x}}$ & $141.00(10)$ \\
\hline $\mathrm{Si} 3^{\mathrm{v}}-\mathrm{Cs} 3-\mathrm{O} 6^{\mathrm{x}}$ & $112.22(6)$ & $\mathrm{Cs} 3-\mathrm{O} 7-\mathrm{Cs} 2^{\mathrm{x}}$ & $64.24(6)$ \\
\hline $\mathrm{Lu} 1-\mathrm{Cs} 3-\mathrm{O} 6^{\mathrm{x}}$ & $73.34(6)$ & $\mathrm{Cs} 1^{\mathrm{x}}-\mathrm{O} 7-\mathrm{Cs} 2^{\mathrm{x}}$ & $48.17(4)$ \\
\hline $\mathrm{Si} 1^{\mathrm{iv}}-\mathrm{Cs} 3-\mathrm{O}^{\mathrm{x}}$ & $131.56(7)$ & $\mathrm{Cs} 2^{\mathrm{vii}}-\mathrm{O} 7-\mathrm{Cs} 2^{\mathrm{x}}$ & $88.32(7)$ \\
\hline $\mathrm{Cs} 2^{\mathrm{xi}}-\mathrm{Cs} 3-\mathrm{O} 6^{\mathrm{x}}$ & $78.22(6)$ & $\mathrm{Cs} 3^{\mathrm{xiii}}-\mathrm{O} 7-\mathrm{Cs} 2^{\mathrm{x}}$ & $100.28(7)$ \\
\hline $\mathrm{Cs} 2-\mathrm{Cs} 3-\mathrm{O} 6^{\mathrm{x}}$ & $154.60(6)$ & $\mathrm{Si} 3-\mathrm{O} 7-\mathrm{Cs} 1^{\mathrm{xiv}}$ & $145.31(19)$ \\
\hline $\mathrm{O} 1-\mathrm{Cs} 3-\mathrm{O} 7^{\mathrm{xi}}$ & $142.13(10)$ & Sil-O7-Cs1 $1^{\text {xiv }}$ & $78.16(15)$ \\
\hline $\mathrm{O} 9^{\mathrm{iv}}-\mathrm{Cs} 3-\mathrm{O}^{\mathrm{xi}}$ & $98.13(10)$ & $\mathrm{Cs} 2-\mathrm{O} 7-\mathrm{Cs}^{1}{ }^{\mathrm{xiv}}$ & $55.03(6)$ \\
\hline $\mathrm{O} 5-\mathrm{Cs} 3-\mathrm{O} 7^{\mathrm{xi}}$ & $106.53(10)$ & $\mathrm{Cs} 1^{\mathrm{vii}}-\mathrm{O} 7-\mathrm{Cs} 1^{\mathrm{xiv}}$ & $106.55(9)$ \\
\hline $\mathrm{O} 4-\mathrm{Cs} 3-\mathrm{O} 7^{\mathrm{xi}}$ & $79.38(10)$ & $\mathrm{Cs} 3^{\text {viii }}-\mathrm{O} 7-\mathrm{Cs} 1^{\mathrm{xiv}}$ & $50.44(4)$ \\
\hline $\mathrm{O} 3^{\mathrm{ix}}-\mathrm{Cs} 3-07^{\mathrm{xi}}$ & $118.35(10)$ & $\mathrm{Cs} 3-\mathrm{O} 7-\mathrm{Cs} 1^{\mathrm{xiv}}$ & $74.63(6)$ \\
\hline $\mathrm{O} 8-\mathrm{Cs} 3-\mathrm{O} 7^{\mathrm{xi}}$ & $100.78(9)$ & $\mathrm{Cs} 1^{\mathrm{x}}-\mathrm{O} 7-\mathrm{Cs} 1^{\mathrm{xiv}}$ & $125.86(9)$ \\
\hline $\mathrm{O} 6-\mathrm{Cs} 3-\mathrm{O} 7^{\mathrm{xi}}$ & $105.00(8)$ & $\mathrm{Cs} 2^{\mathrm{vii}}-\mathrm{O} 7-\mathrm{Cs} 1^{\mathrm{xiv}}$ & $84.31(6)$ \\
\hline $\mathrm{Cs} 1^{\mathrm{x}}-\mathrm{Cs} 3-\mathrm{O} 7^{\mathrm{xi}}$ & $54.57(6)$ & $\mathrm{Cs} 3^{\mathrm{xiii}}-\mathrm{O} 7-\mathrm{Cs} 1^{\mathrm{xiv}}$ & $157.05(9)$ \\
\hline $\mathrm{Si} 3^{\mathrm{v}}-\mathrm{Cs} 3-\mathrm{O}^{\mathrm{xi}}$ & $135.48(6)$ & $\mathrm{Cs} 2^{\mathrm{x}}-\mathrm{O} 7-\mathrm{Cs} 1^{\mathrm{xiv}}$ & $101.13(7)$ \\
\hline $\mathrm{Lu} 1-\mathrm{Cs} 3-\mathrm{O} 7^{\mathrm{xi}}$ & $112.19(6)$ & $\mathrm{Si} 3-\mathrm{O} 7-\mathrm{Cs} 3^{\mathrm{vii}}$ & $122.73(17)$ \\
\hline $\mathrm{Si} 1^{\mathrm{iv}}-\mathrm{Cs} 3-\mathrm{O} 7^{\mathrm{xi}}$ & $93.14(6)$ & $\mathrm{Si} 1-\mathrm{O} 7-\mathrm{Cs} 3^{\mathrm{vii}}$ & $21.86(12)$ \\
\hline $\mathrm{Cs} 2^{\mathrm{xi}}-\mathrm{Cs} 3-\mathrm{O} 7^{\mathrm{xi}}$ & $48.82(6)$ & $\mathrm{Cs} 2-\mathrm{O} 7-\mathrm{Cs} 3^{\mathrm{vii}}$ & $145.54(11)$ \\
\hline $\mathrm{Cs} 2-\mathrm{Cs} 3-\mathrm{O}^{\mathrm{xi}}$ & $150.34(6)$ & $\mathrm{Cs} 1^{\mathrm{vii}}-\mathrm{O} 7-\mathrm{Cs} 3^{\mathrm{vii}}$ & $89.11(8)$ \\
\hline $\mathrm{O} 6^{\mathrm{x}}-\mathrm{Cs} 3-\mathrm{O} 7^{\mathrm{xi}}$ & $38.86(8)$ & $\mathrm{Cs} 3^{\text {viii }}-\mathrm{O} 7-\mathrm{Cs} 3^{\text {vii }}$ & $103.78(8)$ \\
\hline $\mathrm{O} 1-\mathrm{Cs} 3-\mathrm{Cs} 1^{\mathrm{ix}}$ & $69.56(8)$ & $\mathrm{Cs} 3-\mathrm{O} 7-\mathrm{Cs} 3^{\mathrm{vii}}$ & $102.51(8)$ \\
\hline $\mathrm{O} 9^{\mathrm{iv}}-\mathrm{Cs} 3-\mathrm{Cs} 1^{\mathrm{ix}}$ & $84.40(8)$ & $\mathrm{Cs} 1^{\mathrm{x}}-\mathrm{O} 7-\mathrm{Cs} 3^{\text {vii }}$ & $88.34(7)$ \\
\hline $\mathrm{O} 5-\mathrm{Cs} 3-\mathrm{Cs} 1^{\mathrm{ix}}$ & $106.18(8)$ & $\mathrm{Cs} 2^{\mathrm{vii}}-\mathrm{O} 7-\mathrm{Cs} 3^{\mathrm{vii}}$ & $44.34(4)$ \\
\hline $\mathrm{O} 4-\mathrm{Cs} 3-\mathrm{Cs} 1^{\mathrm{ix}}$ & $47.54(8)$ & $\mathrm{Cs} 3^{\mathrm{xiii}}-\mathrm{O} 7-\mathrm{Cs} 3^{\mathrm{vii}}$ & $97.92(7)$ \\
\hline $\mathrm{O} 3^{\mathrm{ix}}-\mathrm{Cs} 3-\mathrm{Cs} 1^{\mathrm{ix}}$ & $44.94(8)$ & $\mathrm{Cs} 2^{\mathrm{x}}-\mathrm{O} 7-\mathrm{Cs} 3^{\text {vii }}$ & $44.05(3)$ \\
\hline $\mathrm{O} 8-\mathrm{Cs} 3-\mathrm{Cs} 1^{\mathrm{ix}}$ & $174.19(7)$ & $\mathrm{Cs} 1^{\mathrm{xiv}}-\mathrm{O} 7-\mathrm{Cs} 3^{\mathrm{vii}}$ & $91.78(6)$ \\
\hline $\mathrm{O} 6-\mathrm{Cs} 3-\mathrm{Cs} 1^{\mathrm{ix}}$ & $130.72(6)$ & $\mathrm{Si} 2-\mathrm{O} 8-\mathrm{Si} 3$ & $132.9(2)$ \\
\hline $\mathrm{Cs} 1^{\mathrm{x}}-\mathrm{Cs} 3-\mathrm{Cs} 1^{\mathrm{ix}}$ & $131.185(15)$ & $\mathrm{Si} 2-\mathrm{O} 8-\mathrm{Cs} 1^{\mathrm{x}}$ & $110.77(19)$ \\
\hline $\mathrm{Si} 3^{\mathrm{v}}-\mathrm{Cs} 3-\mathrm{Cs} 1^{\mathrm{ix}}$ & $55.41(2)$ & $\mathrm{Si} 3-\mathrm{O} 8-\mathrm{Cs} 1^{\mathrm{x}}$ & $93.66(16)$ \\
\hline $\mathrm{Lu} 1-\mathrm{Cs} 3-\mathrm{Cs} 1^{\mathrm{ix}}$ & $71.536(10)$ & $\mathrm{Si} 2-\mathrm{O} 8-\mathrm{Cs} 2$ & $93.67(16)$ \\
\hline $\mathrm{Si} 1^{\mathrm{iv}}-\mathrm{Cs} 3-\mathrm{Cs} 1^{\mathrm{ix}}$ & $106.66(3)$ & $\mathrm{Si} 3-\mathrm{O} 8-\mathrm{Cs} 2$ & $92.90(17)$ \\
\hline $\mathrm{Cs} 2^{\mathrm{xi}}-\mathrm{Cs} 3-\mathrm{Cs} 1^{\mathrm{ix}}$ & $64.613(12)$ & $\mathrm{Cs} 1{ }^{\mathrm{x}}-\mathrm{O} 8-\mathrm{Cs} 2$ & $138.75(13)$ \\
\hline $\mathrm{Cs} 2-\mathrm{Cs} 3-\mathrm{Cs} 1^{\mathrm{ix}}$ & $126.802(14)$ & $\mathrm{Si} 2-\mathrm{O} 8-\mathrm{Cs} 3$ & $106.38(17)$ \\
\hline $\mathrm{O} 6^{\mathrm{x}}-\mathrm{Cs} 3-\mathrm{Cs} 1^{\mathrm{ix}}$ & $67.30(6)$ & $\mathrm{Si} 3-\mathrm{O} 8-\mathrm{Cs} 3$ & $120.04(18)$ \\
\hline $\mathrm{O} 7^{\mathrm{xi}}-\mathrm{Cs} 3-\mathrm{Cs} 1^{\mathrm{ix}}$ & $80.07(6)$ & $\mathrm{Cs} 1 \times-\mathrm{O} 8-\mathrm{Cs} 3$ & $68.85(8)$ \\
\hline $\mathrm{O} 1-\mathrm{Cs} 3-\mathrm{O} 7$ & $79.30(10)$ & $\mathrm{Cs} 2-\mathrm{O} 8-\mathrm{Cs} 3$ & $72.67(8)$ \\
\hline
\end{tabular}




\begin{tabular}{|c|c|}
\hline $\mathrm{O} 9^{\text {iv }}-\mathrm{Cs} 3-\mathrm{O} 7$ & $118.83(10)$ \\
\hline $\mathrm{O} 5-\mathrm{Cs} 3-\mathrm{O} 7$ & $41.93(10)$ \\
\hline $\mathrm{O} 4-\mathrm{Cs} 3-\mathrm{O} 7$ & $103.31(9)$ \\
\hline $\mathrm{O} 3^{\mathrm{ix}}-\mathrm{Cs} 3-\mathrm{O} 7$ & $122.38(9)$ \\
\hline $\mathrm{O} 8-\mathrm{Cs} 3-\mathrm{O} 7$ & $39.81(9)$ \\
\hline $\mathrm{O} 6-\mathrm{Cs} 3-\mathrm{O} 7$ & $76.39(8)$ \\
\hline $\mathrm{Cs} 11^{\mathrm{x}}-\mathrm{Cs} 3-\mathrm{O} 7$ & $65.40(6)$ \\
\hline $\mathrm{Si}^{2}-\mathrm{Cs} 3-\mathrm{O} 7$ & $100.27(6)$ \\
\hline $\mathrm{Lu} 1-\mathrm{Cs} 3-\mathrm{O} 7$ & $73.32(6)$ \\
\hline $\mathrm{Si} 1^{\mathrm{iv}}-\mathrm{Cs} 3-\mathrm{O} 7$ & $102.24(6)$ \\
\hline $\mathrm{Cs} 2^{\mathrm{xi}}-\mathrm{Cs} 3-\mathrm{O} 7$ & $150.75(6)$ \\
\hline $\mathrm{Cs} 2-\mathrm{Cs} 3-\mathrm{O} 7$ & $48.17(6)$ \\
\hline $\mathrm{O} 6^{\mathrm{x}}-\mathrm{Cs} 3-\mathrm{O} 7$ & $107.28(8)$ \\
\hline $\mathrm{O} 7{ }^{x i}-\mathrm{Cs} 3-\mathrm{O} 7$ & $118.79(10)$ \\
\hline $\mathrm{Cs} 1^{\mathrm{ix}}-\mathrm{Cs} 3-\mathrm{O} 7$ & $144.45(6)$ \\
\hline $\mathrm{O} 1-\mathrm{Cs} 3-\mathrm{Si}^{\mathrm{x}}$ & $83.37(8)$ \\
\hline $\mathrm{O} 9^{\mathrm{iv}}-\mathrm{Cs} 3-\mathrm{Si}^{\mathrm{x}}$ & $131.40(9)$ \\
\hline $\mathrm{O} 5-\mathrm{Cs} 3-\mathrm{Si} 2^{\mathrm{x}}$ & $69.40(9)$ \\
\hline $\mathrm{O} 4-\mathrm{Cs} 3-\mathrm{Si} 2^{\mathrm{x}}$ & $19.82(8)$ \\
\hline $\mathrm{O}^{\mathrm{ix}}-\mathrm{Cs} 3-\mathrm{Si} 2^{\mathrm{x}}$ & $92.60(8)$ \\
\hline $\mathrm{O} 8-\mathrm{Cs} 3-\mathrm{Si} 2^{\mathrm{x}}$ & $133.96(7)$ \\
\hline $\mathrm{O} 6-\mathrm{Cs} 3-\mathrm{Si} 2^{\mathrm{x}}$ & $164.69(6)$ \\
\hline $\mathrm{Cs} 1^{x}-\mathrm{Cs} 3-\mathrm{Si}^{\mathrm{x}}$ & 87.07 (2) \\
\hline $\mathrm{Si} 3^{\mathrm{v}}-\mathrm{Cs} 3-\mathrm{Si} 2^{\mathrm{x}}$ & $88.85(3)$ \\
\hline $\mathrm{Lu} 1-\mathrm{Cs} 3-\mathrm{Si} 2^{\mathrm{x}}$ & $54.30(2)$ \\
\hline $\mathrm{Si} 1^{\mathrm{iv}}-\mathrm{Cs} 3-\mathrm{Si} 2^{\mathrm{x}}$ & $145.32(3)$ \\
\hline $\mathrm{Cs} 2^{\mathrm{xi}}-\mathrm{Cs} 3-\mathrm{Si}^{2}{ }^{\mathrm{x}}$ & $87.09(2)$ \\
\hline $\mathrm{Cs} 2-\mathrm{Cs} 3-\mathrm{Si} 2^{\mathrm{x}}$ & $144.67(2)$ \\
\hline $\mathrm{O} 6^{\mathrm{x}}-\mathrm{Cs} 3-\mathrm{Si}^{\mathrm{x}}$ & $23.68(6)$ \\
\hline $\mathrm{O} 7^{\mathrm{xi}}-\mathrm{Cs} 3-\mathrm{Si} 2^{\mathrm{x}}$ & $59.69(6)$ \\
\hline $\mathrm{Cs} 1^{1 \mathrm{x}}-\mathrm{Cs} 3-\mathrm{Si}^{2}{ }^{\mathrm{x}}$ & $51.38(2)$ \\
\hline $\mathrm{O} 7-\mathrm{Cs} 3-\mathrm{Si} 2^{\mathrm{x}}$ & $109.57(6)$ \\
\hline $\mathrm{O} 1-\mathrm{Cs} 3-\mathrm{Si} 2$ & $121.10(8)$ \\
\hline $\mathrm{O} 9^{\mathrm{iv}}-\mathrm{Cs} 3-\mathrm{Si} 2$ & $68.31(8)$ \\
\hline $\mathrm{O} 5-\mathrm{Cs} 3-\mathrm{Si} 2$ & $101.67(8)$ \\
\hline $\mathrm{O} 4-\mathrm{Cs} 3-\mathrm{Si} 2$ & $159.68(8)$ \\
\hline $\mathrm{O} 3^{\mathrm{ix}}-\mathrm{Cs} 3-\mathrm{Si} 2$ & $118.59(8)$ \\
\hline $\mathrm{O} 8-\mathrm{Cs} 3-\mathrm{Si} 2$ & $22.61(7)$ \\
\hline $\mathrm{O} 6-\mathrm{Cs} 3-\mathrm{Si} 2$ & $23.29(6)$ \\
\hline $\mathrm{Cs} 1^{\mathrm{x}}-\mathrm{Cs} 3-\mathrm{Si} 2$ & $61.02(2)$ \\
\hline $\mathrm{Si} 3^{\mathrm{v}}-\mathrm{Cs} 3-\mathrm{Si} 2$ & $122.23(3)$ \\
\hline $\mathrm{Lu} 1-\mathrm{Cs} 3-\mathrm{Si} 2$ & $133.90(2)$ \\
\hline $\mathrm{Si} 1^{\mathrm{iv}}-\mathrm{Cs} 3-\mathrm{Si} 2$ & $45.30(3)$ \\
\hline $\mathrm{Cs} 2^{\mathrm{xi}}-\mathrm{Cs} 3-\mathrm{Si} 2$ & $91.84(2)$ \\
\hline $\mathrm{Cs} 2-\mathrm{Cs} 3-\mathrm{Si} 2$ & $54.15(2)$ \\
\hline $\mathrm{O} 6^{\mathrm{x}}-\mathrm{Cs} 3-\mathrm{Si} 2$ & $125.41(6)$ \\
\hline $\mathrm{O}^{\mathrm{xi}}-\mathrm{Cs} 3-\mathrm{Si} 2$ & $96.34(6)$ \\
\hline $\mathrm{Cs} 1^{\mathrm{ix}}-\mathrm{Cs} 3-\mathrm{Si} 2$ & $151.80(2)$ \\
\hline
\end{tabular}

\begin{tabular}{|c|c|}
\hline $\mathrm{Si} 2-\mathrm{O} 8-\mathrm{Cs} 2^{\mathrm{xiii}}$ & $67.39(13)$ \\
\hline $\mathrm{Si} 3-\mathrm{O} 8-\mathrm{Cs} 2^{\mathrm{xiii}}$ & $87.19(15)$ \\
\hline $\mathrm{Cs} 1^{\mathrm{x}}-\mathrm{O} 8-\mathrm{Cs} 2^{\mathrm{xiii}}$ & $68.13(7)$ \\
\hline $\mathrm{Cs} 2-\mathrm{O} 8-\mathrm{Cs} 2^{\mathrm{xiii}}$ & $152.92(12)$ \\
\hline $\mathrm{Cs} 3-\mathrm{O} 8-\mathrm{Cs} 2^{\mathrm{xiii}}$ & $129.88(11)$ \\
\hline $\mathrm{Si} 2-\mathrm{O} 8-\mathrm{Cs} 3^{\mathrm{xiii}}$ & $101.07(15)$ \\
\hline $\mathrm{Si} 3-\mathrm{O} 8-\mathrm{Cs} 3^{\mathrm{xiii}}$ & $38.67(11)$ \\
\hline $\mathrm{Cs} 1^{x}-\mathrm{O} 8-\mathrm{Cs} 3^{\mathrm{xiii}}$ & $87.23(8)$ \\
\hline $\mathrm{Cs} 2-\mathrm{O} 8-\mathrm{Cs} 3^{\mathrm{xiii}}$ & $121.08(10)$ \\
\hline $\mathrm{Cs} 3-\mathrm{O} 8-\mathrm{Cs} 3^{\mathrm{xiii}}$ & $148.47(11)$ \\
\hline $\mathrm{Cs} 2^{\mathrm{xiii}}-\mathrm{O} 8-\mathrm{Cs} 3^{\mathrm{xiii}}$ & $49.74(4)$ \\
\hline $\mathrm{Si} 2-\mathrm{O} 8-\mathrm{Cs} 1^{\mathrm{xiii}}$ & $22.24(10)$ \\
\hline $\mathrm{Si} 3-\mathrm{O} 8-\mathrm{Cs} 1^{\mathrm{xiii}}$ & $114.30(16)$ \\
\hline $\mathrm{Cs} 1^{\mathrm{x}}-\mathrm{O} 8-\mathrm{Cs} 1^{\mathrm{xiii}}$ & $104.99(10)$ \\
\hline $\mathrm{Cs} 2-\mathrm{O} 8-\mathrm{Cs} 1^{\mathrm{xiii}}$ & $109.11(9)$ \\
\hline $\mathrm{Cs} 3-\mathrm{O} 8-\mathrm{Cs} 1^{\mathrm{xiii}}$ & $125.51(10)$ \\
\hline $\mathrm{Cs} 2^{\mathrm{xiii}}-\mathrm{O} 8-\mathrm{Cs} 1^{\mathrm{xiii}}$ & $47.69(4)$ \\
\hline $\mathrm{Cs} 3^{\mathrm{xiii}}-\mathrm{O} 8-\mathrm{Cs} 1^{\mathrm{xiii}}$ & $79.36(6)$ \\
\hline $\mathrm{Si} 2-\mathrm{O} 8-\mathrm{Cs} 1^{\mathrm{vii}}$ & $124.34(17)$ \\
\hline $\mathrm{Si} 3-\mathrm{O} 8-\mathrm{Cs} 1^{\mathrm{vii}}$ & $17.66(11)$ \\
\hline $\mathrm{Cs} 1^{\mathrm{x}}-\mathrm{O} 8-\mathrm{Cs} 1^{\mathrm{vii}}$ & $111.01(10)$ \\
\hline $\mathrm{Cs} 2-\mathrm{O} 8-\mathrm{Cs} 1^{\mathrm{vii}}$ & $77.66(8)$ \\
\hline $\mathrm{Cs} 3-\mathrm{O} 8-\mathrm{Cs} 1^{\mathrm{vii}}$ & $122.06(10)$ \\
\hline $\mathrm{Cs} 2^{\mathrm{xiii}}-\mathrm{O} 8-\mathrm{Cs} 1^{\mathrm{vii}}$ & $96.71(7)$ \\
\hline $\mathrm{Cs} 3^{\mathrm{xiii}}-\mathrm{O} 8-\mathrm{Cs} 1^{\mathrm{vii}}$ & $47.13(4)$ \\
\hline $\mathrm{Cs} 1^{\mathrm{xiii}}-\mathrm{O} 8-\mathrm{Cs} 1^{\mathrm{vii}}$ & $110.80(7)$ \\
\hline $\mathrm{Si} 2-\mathrm{O} 8-\mathrm{Cs} 3^{\mathrm{iii}}$ & $36.07(12)$ \\
\hline $\mathrm{Si} 3-\mathrm{O} 8-\mathrm{Cs} 3^{3 \mathrm{ii}}$ & $111.34(16)$ \\
\hline $\mathrm{Cs} 1^{x}-\mathrm{O} 8-\mathrm{Cs} 3^{\mathrm{iii}}$ & $146.83(11)$ \\
\hline $\mathrm{Cs} 2-\mathrm{O} 8-\mathrm{Cs} 3^{\mathrm{iii}}$ & $63.85(6)$ \\
\hline $\mathrm{Cs} 3-\mathrm{O} 8-\mathrm{Cs} 3^{\mathrm{iii}}$ & $112.60(9)$ \\
\hline $\mathrm{Cs} 2^{\mathrm{xiii}}-\mathrm{O} 8-\mathrm{Cs} 3^{\mathrm{iii}}$ & $90.91(7)$ \\
\hline $\mathrm{Cs} 3^{\mathrm{xiii}}-\mathrm{O} 8-\mathrm{Cs} 3^{\mathrm{iii}}$ & $98.70(6)$ \\
\hline $\mathrm{Cs} 1^{\mathrm{xiii}}-\mathrm{O} 8-\mathrm{Cs} 3^{\mathrm{iii}}$ & $45.48(3)$ \\
\hline $\mathrm{Cs} 1^{\mathrm{vii}}-\mathrm{O} 8-\mathrm{Cs} 3^{\mathrm{iii}}$ & $96.27(6)$ \\
\hline Si1-O9-Lu1 $1^{\text {xiii }}$ & $137.7(2)$ \\
\hline $\mathrm{Si} 1-\mathrm{O} 9-\mathrm{Cs} 3^{\mathrm{vii}}$ & $107.17(19)$ \\
\hline $\mathrm{Lu} 1^{\mathrm{xiii}}-\mathrm{O} 9-\mathrm{Cs} 3^{\mathrm{vii}}$ & $111.71(15)$ \\
\hline $\mathrm{Si} 1-\mathrm{O} 9-\mathrm{Cs} 2^{\mathrm{x}}$ & $99.43(19)$ \\
\hline Lu1 $1^{x i i}-O 9-C s 2^{x}$ & $103.89(15)$ \\
\hline $\mathrm{Cs} 3^{\mathrm{vii}}-\mathrm{O} 9-\mathrm{Cs} 2^{\mathrm{x}}$ & $78.56(10)$ \\
\hline $\mathrm{Si} 1-\mathrm{O} 9-\mathrm{Cs} 1^{\mathrm{x}}$ & $87.21(16)$ \\
\hline Lu1 $1^{\mathrm{xii}}-\mathrm{O} 9-\mathrm{Cs} 1^{\mathrm{x}}$ & $73.42(11)$ \\
\hline $\mathrm{Cs} 3^{\mathrm{vii}}-\mathrm{O} 9-\mathrm{Cs} 1^{\mathrm{x}}$ & $140.36(14)$ \\
\hline $\mathrm{Cs} 2^{\mathrm{x}}-\mathrm{O} 9-\mathrm{Cs} 1^{\mathrm{x}}$ & $62.49(8)$ \\
\hline $\mathrm{Si} 1-\mathrm{O} 9-\mathrm{Cs} 2^{\mathrm{vii}}$ & $82.40(17)$ \\
\hline $\mathrm{Lu} 1^{\mathrm{xiii}}-\mathrm{O} 9-\mathrm{Cs} 2^{\mathrm{vii}}$ & $101.61(14)$ \\
\hline $\mathrm{Cs} 3^{\mathrm{vii}}-\mathrm{O} 9-\mathrm{Cs} 2^{\mathrm{vii}}$ & $61.77(8)$ \\
\hline
\end{tabular}




\begin{tabular}{|c|c|c|c|}
\hline $\mathrm{O} 7-\mathrm{Cs} 3-\mathrm{Si} 2$ & $61.14(6)$ & $\mathrm{Cs} 2^{\mathrm{x}}-\mathrm{O} 9-\mathrm{Cs} 2^{\mathrm{vii}}$ & $138.56(12)$ \\
\hline $\mathrm{Si} 2^{\mathrm{x}}-\mathrm{Cs} 3-\mathrm{Si} 2$ & $148.01(2)$ & $\mathrm{Cs} 1^{\mathrm{x}}-\mathrm{O} 9-\mathrm{Cs} 2^{\mathrm{vii}}$ & $157.83(11)$ \\
\hline $\mathrm{O} 1-\mathrm{Cs} 3-\mathrm{Si} 1$ & $72.06(8)$ & $\mathrm{Si} 1-\mathrm{O} 9-\mathrm{Cs} 1^{\mathrm{vii}}$ & $73.68(16)$ \\
\hline $\mathrm{O} 9^{\mathrm{iv}}-\mathrm{Cs} 3-\mathrm{Si} 1$ & $141.23(9)$ & $\mathrm{Lu} 1^{\mathrm{xiii}}-\mathrm{O} 9-\mathrm{Cs} 1^{\mathrm{vii}}$ & $76.55(11)$ \\
\hline $\mathrm{O} 5-\mathrm{Cs} 3-\mathrm{Si} 1$ & $18.98(8)$ & $\mathrm{Cs} 3^{\mathrm{vii}}-\mathrm{O} 9-\mathrm{Cs} 1^{\mathrm{vii}}$ & $112.61(12)$ \\
\hline $\mathrm{O} 4-\mathrm{Cs} 3-\mathrm{Si} 1$ & $80.28(8)$ & $\mathrm{Cs} 2^{\mathrm{x}}-\mathrm{O} 9-\mathrm{Cs} 1^{\mathrm{vii}}$ & $168.03(12)$ \\
\hline $\mathrm{O} 3^{\mathrm{ix}}-\mathrm{Cs} 3-\mathrm{Si} 1$ & $122.08(7)$ & $\mathrm{Cs} 1^{\mathrm{x}}-\mathrm{O} 9-\mathrm{Cs} 1^{\mathrm{vii}}$ & $106.83(9)$ \\
\hline $\mathrm{O} 8-\mathrm{Cs} 3-\mathrm{Si} 1$ & $60.36(7)$ & $\mathrm{Cs} 2^{\mathrm{vii}}-\mathrm{O} 9-\mathrm{Cs} 1^{\mathrm{vii}}$ & $51.41(5)$ \\
\hline $\mathrm{O} 6-\mathrm{Cs} 3-\mathrm{Si} 1$ & $99.67(6)$ & $\mathrm{Si} 1-\mathrm{O} 9-\mathrm{Cs} 1^{\mathrm{xvi}}$ & $154.9(2)$ \\
\hline $\mathrm{Cs} 1^{\mathrm{x}}-\mathrm{Cs} 3-\mathrm{Si} 1$ & $66.26(2)$ & Lu1 $1^{x i i i}-O 9-C s 1^{x v i}$ & $66.01(10)$ \\
\hline $\mathrm{Si} 3^{\mathrm{v}}-\mathrm{Cs} 3-\mathrm{Si} 1$ & $96.60(3)$ & $\mathrm{Cs} 3^{\mathrm{vii}}-\mathrm{O} 9-\mathrm{Cs} 1^{\mathrm{xvi}}$ & $56.91(6)$ \\
\hline $\mathrm{Lu} 1-\mathrm{Cs} 3-\mathrm{Si} 1$ & $54.03(2)$ & $\mathrm{Cs} 2^{\mathrm{x}}-\mathrm{O} 9-\mathrm{Cs} 1^{\mathrm{xvi}}$ & $60.38(7)$ \\
\hline $\mathrm{Si}^{\mathrm{iv}}-\mathrm{Cs} 3-\mathrm{Si} 1$ & $125.54(3)$ & $\mathrm{Cs} 1^{\mathrm{x}}-\mathrm{O} 9-\mathrm{Cs} 1^{\mathrm{xvi}}$ & $94.98(8)$ \\
\hline $\mathrm{Cs} 2^{\mathrm{xi}}-\mathrm{Cs} 3-\mathrm{Si} 1$ & $158.23(2)$ & $\mathrm{Cs} 2^{\mathrm{vii}}-\mathrm{O} 9-\mathrm{Cs} 1^{\mathrm{xvi}}$ & $102.73(8)$ \\
\hline $\mathrm{Cs} 2-\mathrm{Cs} 3-\mathrm{Si} 1$ & $66.87(2)$ & $\mathrm{Cs} 1^{\mathrm{vii}}-\mathrm{O} 9-\mathrm{Cs} 1^{\mathrm{xvi}}$ & $128.74(9)$ \\
\hline $\mathrm{O} 6^{\mathrm{x}}-\mathrm{Cs} 3-\mathrm{Si} 1$ & $87.73(6)$ & $\mathrm{Si} 1-\mathrm{O} 9-\mathrm{Cs} 3$ & $45.19(13)$ \\
\hline $\mathrm{O} 7 \mathrm{xi}-\mathrm{Cs} 3-\mathrm{Si} 1$ & $110.63(6)$ & $\mathrm{Lu}^{\mathrm{xiii}}-\mathrm{O} 9-\mathrm{Cs} 3$ & $115.62(13)$ \\
\hline $\mathrm{Cs} 1^{1 \mathrm{x}}-\mathrm{Cs} 3-\mathrm{Si} 1$ & $124.85(2)$ & $\mathrm{Cs} 3^{\mathrm{vii}}-\mathrm{O} 9-\mathrm{Cs} 3$ & $126.33(12)$ \\
\hline $\mathrm{O} 7-\mathrm{Cs} 3-\mathrm{Si} 1$ & $23.37(6)$ & $\mathrm{Cs} 2^{\mathrm{x}}-\mathrm{O} 9-\mathrm{Cs} 3$ & $67.01(7)$ \\
\hline $\mathrm{Si} 2^{\mathrm{x}}-\mathrm{Cs} 3-\mathrm{Si} 1$ & $86.50(3)$ & $\mathrm{Cs} 1{ }^{\mathrm{x}}-\mathrm{O} 9-\mathrm{Cs} 3$ & $44.85(4)$ \\
\hline $\mathrm{Si} 2-\mathrm{Cs} 3-\mathrm{Si} 1$ & $82.77(3)$ & $\mathrm{Cs} 2^{\mathrm{vii}}-\mathrm{O} 9-\mathrm{Cs} 3$ & $127.56(9)$ \\
\hline $\mathrm{O} 1-\mathrm{Cs} 3-\mathrm{Lu} 1^{\mathrm{i}}$ & $75.95(8)$ & $\mathrm{Cs} 1^{\mathrm{vii}}-\mathrm{O} 9-\mathrm{Cs} 3$ & $101.81(7)$ \\
\hline $\mathrm{O} 9^{\mathrm{iv}}-\mathrm{Cs} 3-\mathrm{Lu} 1^{\mathrm{i}}$ & $28.40(8)$ & $\mathrm{Cs} 1^{\mathrm{xvi}}-\mathrm{O} 9-\mathrm{Cs} 3$ & $125.13(9)$ \\
\hline $\mathrm{O} 5-\mathrm{Cs} 3-\mathrm{Lu} 1^{\mathrm{i}}$ & $138.58(8)$ & $\mathrm{Si} 1-\mathrm{O} 9-\mathrm{Cs} 3^{\mathrm{xiii}}$ & $103.09(17)$ \\
\hline $\mathrm{O} 4-\mathrm{Cs} 3-\mathrm{Lu} 1^{\mathrm{i}}$ & $107.15(8)$ & Lu1 $1^{\text {iii }}-09-\mathrm{Cs} 3^{\text {xiii }}$ & $35.53(7)$ \\
\hline $\mathrm{O} 3^{\mathrm{ix}}-\mathrm{Cs} 3-\mathrm{Lu} 1^{\mathrm{i}}$ & $30.07(7)$ & $\mathrm{Cs} 3^{\mathrm{vii}}-\mathrm{O} 9-\mathrm{Cs} 3^{\mathrm{xiii}}$ & $135.25(12)$ \\
\hline $\mathrm{O} 8-\mathrm{Cs} 3-\mathrm{Lu} 1^{\mathrm{i}}$ & $111.64(7)$ & $\mathrm{Cs} 2^{\mathrm{x}}-\mathrm{O} 9-\mathrm{Cs} 3^{\mathrm{xiii}}$ & $127.78(11)$ \\
\hline $\mathrm{O} 6-\mathrm{Cs} 3-\mathrm{Lu} 1^{\mathrm{i}}$ & $69.75(6)$ & $\mathrm{Cs} 1^{\mathrm{x}}-\mathrm{O} 9-\mathrm{Cs} 3^{\mathrm{xiii}}$ & $72.23(6)$ \\
\hline $\mathrm{Cs} 1^{\mathrm{x}}-\mathrm{Cs} 3-\mathrm{Lu} 1^{\mathrm{i}}$ & $144.048(14)$ & $\mathrm{Cs} 2^{\mathrm{vii}}-\mathrm{O} 9-\mathrm{Cs} 3^{\mathrm{xiii}}$ & $91.08(8)$ \\
\hline $\mathrm{Si} 3^{\mathrm{v}}-\mathrm{Cs} 3-\mathrm{Lu} 1^{\mathrm{i}}$ & $52.12(2)$ & $\mathrm{Cs} 1^{\mathrm{vii}}-\mathrm{O} 9-\mathrm{Cs} 3^{\mathrm{xiii}}$ & $47.25(4)$ \\
\hline $\mathrm{Lu} 1-\mathrm{Cs} 3-\mathrm{Lu} 1^{\mathrm{i}}$ & $108.890(9)$ & $\mathrm{Cs} 1^{\mathrm{xvi}}-\mathrm{O} 9-\mathrm{Cs} 3^{\mathrm{xii}}$ & $101.38(7)$ \\
\hline $\mathrm{Si} 1^{\mathrm{iv}}-\mathrm{Cs} 3-\mathrm{Lu} 1^{\mathrm{i}}$ & $51.75(2)$ & $\mathrm{Cs} 3-\mathrm{O} 9-\mathrm{Cs} 3^{\mathrm{xiii}}$ & $98.38(7)$ \\
\hline $\mathrm{Cs} 2^{\mathrm{xi}}-\mathrm{Cs} 3-\mathrm{Lu} 1^{\mathrm{i}}$ & $61.779(9)$ & & \\
\hline
\end{tabular}

Symmetry codes: (i) $-x,-y+1, z+1 / 2$; (ii) $-x,-y, z+1 / 2$; (iii) $-x+1 / 2, y-1 / 2, z+1 / 2$; (iv) $-x+1 / 2, y+1 / 2, z+1 / 2$; (v) $x-1 / 2,-y+1 / 2, z$; (vi) $x, y, z+1$; (vii) $-x+1 / 2, y-1 / 2, z-1 / 2$; (viii) $x, y-1, z$; (ix) $-x,-y+1, z-1 / 2$; (x) $-x+1 / 2, y+1 / 2, z-1 / 2$; (xi) $x, y+1, z$; (xii) $x, y, z-1$; (xiii) $x+1 / 2,-y+1 / 2, z$; (xiv) $-x,-y$, $z-1 / 2$; (xv) $x, y+1, z-1 ;(\mathrm{xvi}) x+1 / 2,-y+1 / 2, z-1$. 